\title{
Bond-operator theory of doped antiferromagnets: From Mott insulators with bond-centered charge order to superconductors with nodal fermions
}

\section{Citation}

Park, Kwon, and Subir Sachdev. 2001. "Bond-Operator Theory of Doped Antiferromagnets: From Mott Insulators with Bond-Centered Charge Order to Superconductors with Nodal Fermions." Physical Review B 64 (18). https://doi.org/10.1103/physrevb.64.184510.

\section{Permanent link}

http://nrs.harvard.edu/urn-3:HUL.InstRepos:41417432

\section{Terms of Use}

This article was downloaded from Harvard University's DASH repository, and is made available under the terms and conditions applicable to Other Posted Material, as set forth at http:// nrs.harvard.edu/urn-3:HUL.InstRepos:dash.current.terms-of-use\#LAA

\section{Share Your Story}

The Harvard community has made this article openly available. Please share how this access benefits you. Submit a story.

Accessibility 


\title{
Bond operator theory of doped antiferromagnets: from Mott insulators with bond-centered charge order, to superconductors with nodal fermions
}

\author{
Kwon Park ${ }^{1}$ and Subir Sachdev ${ }^{1,2}$ \\ ${ }^{1}$ Department of Physics, Yale University, P.O. Box 208120, New Haven, CT 06520-8120 \\ ${ }^{2}$ Department of Physics, Harvard University, Cambridge MA 02138
}

(August 1, 2001)

\begin{abstract}
The ground states and excitations of two-dimensional insulating and doped Mott insulators are described by a bond operator formalism. While the method represents the degrees of freedom of an arbitrary antiferromagnet exactly, it is especially suited to systems in which there is a natural pairing of sites into bonds, as in states with spontaneous or explicit spin-Peierls order (or bond-centered charge order). In the undoped insulator, as discussed previously, we obtain both paramagnetic and magnetically-ordered states. We describe the evolution of superconducting order in the ground state with increasing doping - at low doping, the superconductivity is weak, can co-exist with magnetic order, and there are no gapless spin 1/2 fermionic excitations; at high doping, the magnetic order is absent and we obtain a BCS $d$-wave superconductor with gapless spin $1 / 2$, nodal fermions. We present the critical theory describing the onset of these nodal fermionic excitations. We discuss the evolution of the spin spectrum, and obtain regimes where a spin 1 exciton contributes a sharp resonance in the dynamic spin susceptiblity. We also discuss the experimental consequences of low-energy, dynamically fluctuating, spin-Peierls order in an isotropic $\mathrm{CuO}_{2}$ plane-we compute consequences for the damping and dispersion of an optical phonon involving primarily the $\mathrm{O}$ ions, and compare the results with recent neutron scattering measurements of phonon spectra.
\end{abstract}

\section{INTRODUCTION}

By now, it is reasonably well established that the doped antiferromagnets found in the cuprate compounds have a superconducting ground state with a $d$-wave pairing symmetry. Moreover, many low temperature $(T)$ properties appear to be well described in the framework of the conventional BCS theory of $d$-wave superconductors. There are a number of fascinating properties at temperatures above $T_{c}$ (the critical temperature for the onset of superconductivity) which are not well understood, but there are numerous plausible candidate theories for these, involving crossovers between different competing orders in doped antiferromagnet 1 .

However, there are some low $T$ properties of the superconducting state that do not appear naturally in the traditional BCS framework. Among these are $(a)$ the appearance of a $S=1 / 2$ moment near mon-magnetic Zn or $\mathrm{Li}$ impurties in the underdoped region 2 l $3,(b)$ the presence of low energy collective spin excitations (a $S=1$ spin exciton) at $(\pi, \pi)$ and nearby incommensurate wavevectors, and $(c)$ instabilities to various co-existing spin and charge density wave states. While it is possible to "cook-up" a microscopic Hamiltonian, and a corresponding HartreeFock treatment, to generate any of these physical properties in the superconducting state, a proper understanding of the physics should require that they emerge naturally from some deeper principle.

In a series of papers 4 10, it has been proposed that all of these unusual properties of the superconductor emerge very naturally in a theory of the doped Mott insulator. The theory is best understood in two distinct steps. In the first step, we disrupt the magnetic Néel long-range order in the insulator by adding some frustrating exchange interactions. Then, in step two, we dope the paramagnetic Mott insulator obtained in step one with mobile charge carriers.

Considerable theoretical progress has been made in understanding the first step: the quantum transition involving the destruction of Néel long-range order in the half-filled insulator. It has been argued that the first paramagnetic state on the other side of such a quantum critical point generically has the following properties:

(i) a stable $S=1$ particle excitation (an exciton) and permanent confinement of $S=1 / 2$ spinons,

(ii) broken translational symmetry due to the appearance of a bond-centered charge (e.g. spin-Peierls) order, and (iii) confinement of a $S=1 / 2$ moment in the vicinity of non-magnetic $\mathrm{Zn} / \mathrm{Li}$ impurities.

Note the similarity of the features (i)-(iii) of the insulator to the properties $(a)-(c)$ of the superconductor. The essence of step two is then very simple: properties (i)-(iii) survive for a finite range of doping the paramagnetic Mott insulator, and this provides a "natural" understanding of properties $(a)-(c)$ of the superconductor.

We now present a somewhat more detailed discussion of step two: doping the confined Mott insulator with mobile charge carriers. Apart from a possible insulating Wigner crystal ground states at very low doping (such a Wigner crystal state must appear in the presence of long-range Coulomb interactions) the ground state is expected to be superconducting 5.11 , and this will also be the case in the calculations in the present paper. The pairing amplitude is found to be $d$-wave like $\begin{aligned} & \text { - } \\ & \text { i.e. the }\end{aligned}$ 
pairing amplitude has opposite signs in the horizontal and vertical directions. By adjusting the strength of the Coulomb repulsion between the holes, we can modify the characteristic size of the hole pairs. Only for the larger hole pairs does the superconducting ground state posses gapless nodal fermionic excitations. We discuss the fate of (i)-(iii) in the doped antiferromagnet, along with connections to the experiments in turn:

(i) $S=1$ collective spin exciton: In the confined insulator, the lowest energy excitation above the spin gap is a stable, $S=1$, bosonic exciton with a minimum in its dispersion at $(\pi, \pi)$. Upon doping to the superconductor, additional gapped $S=1 / 2$ fermionic excitations are expected to appear 12.13 (these are the Bogoliubov quasiparticles). For sufficiently large doping, the fermionic spectral becomes gapless at four nodal points in the Brillouin zone, and so the global spin gap vanishes. In general, the $S=1$ boson will be unstable to decay in into two of these fermionic excitations. However, constraints from momentum conservation can (and do) protect the integrity of the $S=1$ exciton in certain regions of the Brillouin zone. In particular, the $S=1$ exciton is likely to be stable near momenta $(\pi, \pi)$; this can happen even if the fermionic excitations are gapless, provided the spacing between the nodal points does not equal $(\pi, \pi)$. Experimentally a $S=1$ neutron scattering resonance is indeed observed in the $d$-wave superconductors 1418 , and it is our contention that this excitation is continuously connected to that in the spin-Peierls insulator. We will obtain explicit results for the evolution of the bosonic and fermionic spin excitations in this paper, from the spin-Peierls insulator, to the superconductor with gapless nodal excitations.

(ii) Broken translational symmetry: In the simplest scenario 5 , which can be realized for a range of couplings in models without long-range Coulomb interactions, the bond-centered charge (or spin Peierls) order of the paramagnetic insulator survives in the doped superconductor all the way upto a critical doping at which full square lattice symmetry is restored in a transition to the $d$-wave superconductor; with Coulomb interactions, Wigner crystal states also appear for a range of very small hole doping, but otherwise the situation is similar. The critical theory of the vanishing of the bond-centered (or site-centered) CDW order in the $d$-wave superconductor has been discussed elsewhered. For other parameters, more complex striped states are also possible, with a period larger than 2 sites and with modulation of the hole density on the sites. Experimentally, chargestripe states of period 4 have been clearly observed 19]20, but it is not yet established whether the modulation of the spin density is site or bond centered. More recently, the observation of McQueeney et al.21 in optimally doped and superconducting LSCO have been interpreted using a picture of bondcentered charge stripes of period 2, like those found in the spin-Peierls state. We will discuss these observations further in Section $\mathrm{V}$. (iii) $S=1 / 2$ moment near $\mathrm{Zn} / \mathrm{Li}$ impurities: In principle, it is possible that the $S=1 / 2$ moment confined near a $\mathrm{Zn} / \mathrm{Li}$ impurity in the paramagnetic Mott insulator disappears immediately at an infinitesimal hole doping concentration: one hole can be trapped near the $\mathrm{Zn} / \mathrm{Li}$ impurity, and this configuration is compatible with the global preservation of the spin-Peierls (or other bond-centered charge-) order. However the kinetic energy cost, makes this unlikely. Barring this uninteresting possibility, the $S=1 / 2$ moment will survive in the superconductor, and this offers a natural explanation for the NMR experimentsl. Eventually, the fermionic $S=1 / 2$ excitatiops of the superconductor will Kondo screen the moment 2224 , but because of the linearly vanishing fermionic density of states at the Fermi level, this happens only above finite values of the impurity exchange coupling and particle-hole asymmetry (i.e. above a critical doping). Moreover, there is no fundamental reason for this Kondo screening transition to co-incide with the point at which translational symmetry is restored (this transition was discussed above in (i)); the two transitions could occur in either order as a function of increasing doping

The purpose of this paper is to present a theory of doped antiferromagents which displays the crossover from an insulator at zero doping (with or without longrange magnetic order) to a superconductor with gapless, nodal, fermionic $S=1 / 2$ excitations at some moderate doping. Further, we require the theory to obey properties (i)-(iii) directly at the mean-field level. While a large number of previous theories of doped antiferromagnets have been presented previously, none of them satisfy all of these requirements. The studies of Ref 7 were able to examine the intricate competition between different charge ordered states and superconductivity - however, the ground states were well away from a region of magnetic order, and there was no sharp, collective, $S=1$ excitation in the Gaussian fluctuations about the meanfield theory. Conversely, approaches which do yield confinement of spinons collective $S=1$ excitations at zero or low doping 12526 are not easily extended to reach a superconducting state with $S=1 / 2$ gapless nodal excitations at moderate doping.

We now outline the remainder of the paper. In Section II we introduce the central formalism of bond operators, and its application to doped antiferromagnets. The main results of bond operator theory of the square lattice antiferromagnet are presented in Section III, with most details of the mean-field calculations being relegated to Appendices B, refsquare, and D: the important phase diagram is in Fig 2. The critical theory for the onset of nodal fermion excitations is in Section IV. Section V differs from the remainder of the paper in that it considers systems in which the bond-centered charge order is not present. Instead it considers the case when the spinPeierls order is dynamically fluctuating and describes its influence on the optical phonon spectrum of the $\mathrm{CuO}_{2}$ 
plane - we find that the calculated phonon spectrm is indeed strikingly similar to recent neutron scattering observations. The main conclusions are stated in Section VI. Finally, in Appendix A we describe the interplay between the spin-Peierls states considered in this paper, and states with electronic "nematic" order.

\section{BOND OPERATORS}

Our approach is a generalization of the bond operator theory of Ref 27 (and the related work of Ref 28) to doped antiferromagnets. The earlier work 27 was designed for insulating systems, and has since been applied succesfully in a number of studies of spin ladder and related compeund 29 36, and also in bilayer quantum Hall systems 37,38. Here we shall extend the formalism to doped antiferromagnets; a closely related extension was discussed by Lee etal.39 but oply applied to one-dimensional systems. Eder 40 , Sushkov4, and Vojta and Becker 42 have also considered doped systems by associated methods. Recently, Jurecka and Brenig 43 have used a bond operator method to analyze a Kondo lattice model using a formulation that bears some similarity to ours.

While our bond operator formalism can, in principle, be applied to an arbitrary doped antiferromagnet, it induces a bias by using a basis of states which explicitly refer to a preferred, disjoint pairing of all the sites. A full and exact solution of the bond operator Hamiltonian should restore the full symmetry of the underlying Hamiltonian in which this preferred pairing may be absent. However, in practice, this restoration of symmetry is difficult to achieve, and this is the principal shortcoming of the bond operator method. So the main utility of the approach lies in treating systems in which there is a natural pairing of sites in the ground state, either imposed by the Hamiltonian, or by a spontaneous symmetry breaking. In this paper, we will restrict consideration to the case where the ground state possesses bond-centered charge density wave (spin-Peierls) order: there is evidently a natural pairing of sites in such a structure. The spinPeierls order can either be spontaneous or explicit; the latter is the case in the doped two-leg ladder compounds like $\mathrm{SrCu}_{2} \mathrm{O}_{3}$ and $\mathrm{Sr}_{14-x} \mathrm{Ca}_{x} \mathrm{Cu}_{24} \mathrm{O}_{41}$. In the presence of this background spin Peierls order, the method can then address the competition between magnetic Néel order and superconductivity, and follow the evolution of the fermionic $S=1 / 2$ and the bosonic $S=1$ excitations.

We will now introduce the formalism by showing the exact mappings between bond and site operators of a pair of sites. In hole-doped antiferromagnets, we can project out all states with 2 electrons on one site, and this leaves a total of 9 states in a pair of sites. Let $c_{1 a}^{\dagger}$ and $c_{2 a}^{\dagger}$ $(a=\uparrow, \downarrow)$ be the electron creation operators on the two sites. Then, as in the insulatore7, we introduce four bond boson creation operators, $s^{\dagger}$ and $t_{\alpha}^{\dagger}(\alpha=x, y, z)$ which are defined by $\left(\sigma_{a b}^{\alpha}\right.$ are the Pauli matrices, and $\varepsilon_{a b}$ is the second-rank antisymmetric tensor with $\varepsilon_{\uparrow \downarrow}=1$ )

$$
\begin{aligned}
& s^{\dagger}|v\rangle=\frac{1}{\sqrt{2}} \varepsilon_{a b} c_{1 a}^{\dagger} c_{2 b}^{\dagger}|0\rangle \\
& t_{\alpha}^{\dagger}|v\rangle=\frac{1}{\sqrt{2}} \sigma_{b c}^{\alpha} \varepsilon_{c a} c_{1 a}^{\dagger} c_{2 b}^{\dagger}|0\rangle
\end{aligned}
$$

where $|0\rangle$ is the electron vacuum i.e. the state with no electrons on the two sites, while $|v\rangle$ is an unphysical state in which none of the bond bosons or fermions are present. To describe the remaining 5 states of the doped antiferromagnet, we introduce the bond fermionic operators $h_{1 a}^{\dagger}$ and $h_{2 a}^{\dagger}$, and the additional bond bosonic operator $d^{\dagger}$ which are defined by

$$
\begin{aligned}
h_{1 a}^{\dagger}|v\rangle & =c_{1 a}^{\dagger}|0\rangle \\
h_{2 a}^{\dagger}|v\rangle & =c_{2 a}^{\dagger}|0\rangle \\
d^{\dagger}|v\rangle & =|0\rangle .
\end{aligned}
$$

The operators $s, d, t_{\alpha}$ all obey the canonical boson commutation relations, while the $h_{1 a}, h_{2 a}$ obey canonical fermion relations. Of course, the total space of states in Fock space of these 5 bosons and 4 fermions is much larger than the 9 states allowed in the doped antiferromagnet. To restrict to the physical subspace we must impose the single constraint

$$
s^{\dagger} s+t_{\alpha}^{\dagger} t_{\alpha}+h_{1 a}^{\dagger} h_{1 a}+h_{2 a}^{\dagger} h_{2 a}+d^{\dagger} d=1 .
$$

In the subspace constrained by (3), we can now write down exact expressions for arbitrary electron operators in terms of the bond operators. First, for the electron spin operators

$$
\begin{aligned}
S_{1 \alpha} & =\frac{1}{2} c_{1 a}^{\dagger} \sigma_{a b}^{\alpha} c_{1 b} \\
S_{2 \alpha} & =\frac{1}{2} c_{2 a}^{\dagger} \sigma_{a b}^{\alpha} c_{2 b},
\end{aligned}
$$

we have the following expressions which generalize those in Ref 27

$$
\begin{aligned}
S_{1 \alpha} & =\frac{1}{2}\left(s^{\dagger} t_{\alpha}+t_{\alpha}^{\dagger} s-i \epsilon_{\alpha \beta \gamma} t_{\beta}^{\dagger} t_{\gamma}\right)+\frac{1}{2} \sigma_{a b}^{\alpha} h_{1 a}^{\dagger} h_{1 b} \\
& \equiv \tilde{S}_{1 \alpha}+\frac{1}{2} \sigma_{a b}^{\alpha} h_{1 a}^{\dagger} h_{1 b} \\
S_{2 \alpha} & =-\frac{1}{2}\left(s^{\dagger} t_{\alpha}+t_{\alpha}^{\dagger} s+i \epsilon_{\alpha \beta \gamma} t_{\beta}^{\dagger} t_{\gamma}\right)+\frac{1}{2} \sigma_{a b}^{\alpha} h_{2 a}^{\dagger} h_{2 b} \\
& \equiv \tilde{S}_{2 \alpha}+\frac{1}{2} \sigma_{a b}^{\alpha} h_{2 a}^{\dagger} h_{2 b},
\end{aligned}
$$

where $\epsilon_{\alpha \beta \gamma}$ is the third-rank antisymmetric tensor with $\epsilon_{x y z}=1$. By considering various matrix elements of the electron creation operators, we can also obtain

$$
\begin{aligned}
& c_{1 a}^{\dagger}=h_{1 a}^{\dagger} d+\frac{1}{\sqrt{2}} \varepsilon_{a b} s^{\dagger} h_{2 b}-\frac{1}{\sqrt{2}} \varepsilon_{a c} \sigma_{c b}^{\alpha} t_{\alpha}^{\dagger} h_{2 b} \\
& c_{2 a}^{\dagger}=h_{2 a}^{\dagger} d+\frac{1}{\sqrt{2}} \varepsilon_{a b} s^{\dagger} h_{1 b}+\frac{1}{\sqrt{2}} \varepsilon_{a c} \sigma_{c b}^{\alpha} t_{\alpha}^{\dagger} h_{1 b} .
\end{aligned}
$$


Indeed, the expression (5) can be obtained (6) after repeated application of the constraint (3). In a similar manner, we can also obtain from (6) (or by direct consideration of matrix elements) the useful expressions

$$
\begin{aligned}
S_{1 \alpha} S_{2 \alpha} & =\tilde{S}_{1 \alpha} \tilde{S}_{2 \alpha}=-\frac{3}{4} s^{\dagger} s+\frac{1}{4} t_{\alpha}^{\dagger} t_{\alpha} \\
c_{1 a}^{\dagger} c_{2 a}+c_{2 a}^{\dagger} c_{1 a} & =h_{1 a}^{\dagger} h_{2 a}+h_{2 a}^{\dagger} h_{1 a} \\
c_{1 a}^{\dagger} c_{1 a} & =1-h_{2 a}^{\dagger} h_{2 a}-d^{\dagger} d \\
c_{2 a}^{\dagger} c_{2 a} & =1-h_{1 a}^{\dagger} h_{1 a}-d^{\dagger} d .
\end{aligned}
$$

We have now completed the definition of the bond operator formalism. By application of (5,6,6) an arbitrary Hamiltonian can be written down in terms of the bond operators. Notice that the right hand sides of all of these equations commute with constraint (3) and so will the resulting Hamiltonian. For most systems of interest, the Hamiltonian will only contain terms which are quartic in the bond operators-we will merely apply the simplest possible Hartree-Fock-BCS theory to solve such a model.

At this point, it is worth pointing an important distinction between the present approach and the familiar "slave" boson and fermion theories. A crucial advantage of our approach is that there are no long-range gauge forces associated with the fluctuations about our simple Hartree-Fock-BCS theory: the $s$ boson is strongly condensed in all the phases, and so the $U(1)$ gauge symmetry associated with (3) is always badly broken. Consequently, the quantum numbers of the bare excitations are also those of the renormalized quasiparticles; this is rarely the case in the slave particle approaches. In particular, in the insulating phases, the main elementary excitation will be the bosonic $t_{\alpha}$ particle; in the paramagnet this is the $S=1$ exciton, while in the Néel state it reduces to the two spin waves. In the superconducting states, we will find that the $t_{\alpha}$ excitations persist as the $S=1$ spin exciton, and the $h_{1 a}, h_{2 a}$ quanta turn into the fermionic, $S=1 / 2$ Bogoliubov quasiparticles. So there is a direct and transparent relationship between the quantum numbers of the bond operators and those of the elementary excitations of all the ground states. This is main benefit of our approach, and we are not aware of any previous theory which has satisfied these criteria. Of course, some of the quantum numbers of our confining theory can also be obtained in a direct Hartree-Fock-BCS theory of an extended Hubbard model of the electrons. However, this has to be followed by an RPA analysis of collective modes to obtain the $S=1$ exciton; moreover, in such theories, the largest energy scale in the problem, the charge gap in the Mott insulator, is unphysically related to the strength of the magnetic or charge order, and is hence grossly underestimated.

We conclude this section by noting how the bond operator theory satisfies criteria (i)-(iii) of Section I. (i) As just noted, the $t_{\alpha}$ quanta are gapped $S=1$ excitons in the insulating paramagnet, and persist as sharp excitations in the superconductor for a finite range of doping.

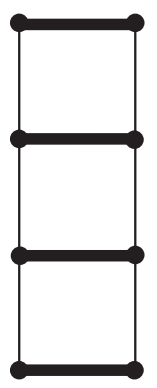

(a)

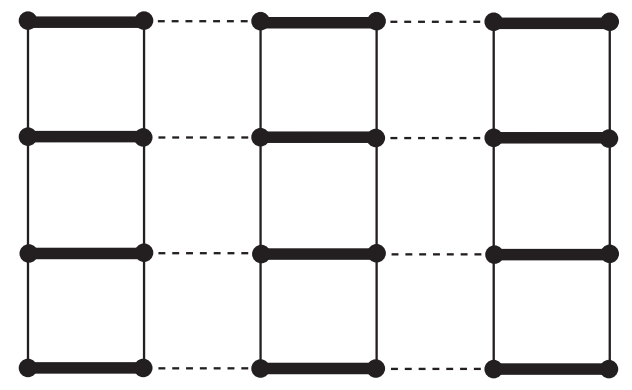

(b)
FIG. 1. Definition of the Hamiltonian $H$ in (8). The two-leg ladder is in (a), while the square lattice is viewed as a set of coupled two-leg ladders in (b). The thick links have $t_{i j}=t, J_{i j}=J, V_{i j}=V$, the thin vertical links have $t_{i j}=t$, $J_{i j}=\lambda J$, and $V_{i j}=V$, while the dashed links have $t_{i j}=t^{\prime}$, $J_{i j}=\lambda^{\prime} J$, and $V_{i j}=V$.

(ii) Any mean-field theory using the bond operator formalism will prefer the bonds on which the operators reside over the others, and this naturally leads to a broken lattice symmetry for symmetric Hamiltonians. Indeed, the primary weakness of the bond operator formalism is that there is no simple way to restore this symmetry. (iii) Placing a $\mathrm{Zn} / \mathrm{Li}$ impurity means that the partner of one site has been removed. The excitations of this site (and only this site) therefore cannot be described by the bond operators above: instead we need a fermionic spinor, $h_{a}$, to create the $S=1 / 2$ state with one electron, and a spinless boson, $b$, to represent the hole. At zero doping, the $h_{a}$ particle constitutes the free $S=1 / 2$ moment near the impurity. This particle will be bound near the impurity site for a finite range of doping, and so the moment will persist for a while in the superconductor.

\section{RESULTS}

We applied the bond-operator method to the $t-J-V$ model defined by the Hamiltonian

$$
\begin{aligned}
H=\sum_{\langle i j\rangle}\left[-t_{i j}(\right. & \left.c_{i a}^{\dagger} c_{j a}+c_{j a}^{\dagger} c_{i a}\right)+J_{i j} S_{i \alpha} S_{j \alpha} \\
& \left.+V_{i j} c_{i a}^{\dagger} c_{i a} c_{j b}^{\dagger} c_{j b}\right]-\mu \sum_{i} c_{i a}^{\dagger} c_{i a},
\end{aligned}
$$

where the sum $\langle i j\rangle$ extends over nearest neighbor pairs on a two-leg ladder or a square lattice, and it is implied that all states with two electrons on any site have been projected out. The $t_{i j}$ are the electron hopping matrix elements, the $J_{i j}>0$ are the antiferromagnetic exchange interactions, the $V_{i j}>0$ are nearest neighbor repulsive Coulomb interactions, and $\mu$ is the chemical potential. The values of the $t_{i j}, J_{i j}$ and $V_{i j}$ are indicated in Fig 1 for both the two-leg ladder and the square lattice. For the two-leg ladder we have $t_{i j}=t, J_{i j}=J, V_{i j}=V$ on the horizontal links, and $t_{i j}=t, J_{i j}=\lambda J$, and $V_{i j}=V$ 
on the vertical links. We view the square lattice as a collection of adjacent two-leg ladders: then the couplings on each ladder are the same as before, while on the links between the ladders we have $t_{i j}=t^{\prime}, J_{i j}=\lambda^{\prime} J$, and $V_{i j}=V$. This parameterization is chosen so that in both cases the exchange interaction decouples into disconnected pairs of sites ("dimers") at $\lambda=\lambda^{\prime}=0$. Further, at $\lambda=\lambda^{\prime}=1, H$ has the full symmetry of the square lattice. However, as discussed earlier, our meanfield theory will continue to have a ground state with the symmetry of Fig 1 b even at these values of $\lambda, \lambda^{\prime}$ : this implies the presence of spontaneous bond-centered charge order of period 2 in the ground state.

The calculation proceeds by the substitution of the operator representations in Section [I] into (8), followed by a Hartree-Fock-BCS treatment of all the quartic terms. The procedure is quite lengthy, but the computations are quite similar to those in earlier work. Details of the calculation are presented in Appendices B, C and D.

Here we will discuss the results of such a calculation. All of the phases can be characterized by a specification of the non-zero expectation values of various combinations of the bond operators in the ground state.

All states have the non-zero expectation values

$$
\langle s\rangle \neq 0 ;\left\langle t_{\alpha}^{\dagger} t_{\alpha}\right\rangle \neq 0 ;\left\langle t_{\alpha} t_{\alpha}\right\rangle \neq 0 .
$$

These non-zero values do not break any physical symmetries of the Hamiltonian, and merely serve to break the $\mathrm{U}(1)$ gauge symmetry associated with the constraint (3). As noted earlier, this is fortunate as no long-range gauge forces then appear in the fluctuations about our ground states. In addition, all phases will also have non-zero expectation values of the operators $t_{\alpha}^{\dagger} t_{\alpha}$ and $h_{1,2 a}^{\dagger} h_{1,2 a}$, which do not break any symmetries of the Hamiltonian, and also commute with the constrains (3).

At zero and non-zero doping we have find also find magnetically ordered states. These are characterized by the non-zero expectation value

$$
\left\langle t_{\alpha}\right\rangle \neq 0
$$

so condensation of single $t_{\alpha}$ bosons (which are $S=1$ particles) leads to the appearance of magnetic order. This also implies that the magnetic ordering transition will be described by the field theory a 3-component spinvector order parameter. Further, provided the ordering wavevector is not exactly equal to the spacing between the points of gapless fermionic excitations, the universality class of the transition is the same as that in the $\mathrm{O}(3)$ non-linear sigma model 144 . This is to be contrasted with magnetic ordering transitions in Schwinger boson theories, which are associated with the condensation of $S=1 / 2$ particles, possibly interacting with each other via gauge forces.

The singlet superconducting states have the anomalous expectation values

$$
\langle d\rangle \neq 0 \quad ; \quad \varepsilon_{a b}\left\langle h_{1,2 a} h_{1,2 b}\right\rangle \neq 0 .
$$

The spatial pattern of these anomalous condensates determines the symmetry of the Cooper pair wavefunction, and whether there are any nodal fermionic quasiparticles.

Finally, we also found states with co-existing magnetic order and superconductivity, in which both (10) and (11) hold.

Our main results are summarized in Fig 2. First, let us review the results at $x=0$, in the insulator. Here the calculations are very similar to those already considered in Ref 27. For small $\lambda^{\prime}$, the ground state is an insulating paramagnet: the symmetry of the Hamiltonian and the ground state is that of Fig $1 \mathrm{~b}$, and the $t_{\alpha}$ excitations have an energy gap: these excitations constitute a $S=1$ collective spin resonance (or a $S=1$ exciton). At a critical value of $\lambda^{\prime}$, the excitation gap vanishes, and longrange Néel order sets in.

Now we turn to non-zero doping. In our HartreeFock-BCS mean-field theory, we find that superconductivity appears at any non-zero doping for small $\lambda^{\prime}$. As one of us has discussed in Ref. 7 (along with references to the earlier literature), this is surely an artifact of our approximation at very small $x$ : in the presence of long-range Coulomb interactions, Wigner crystal states will be present for very small hole concentration. Even in our theory without long-range Coulomb interactions, long-range charge inhognogeneities can also appear in our mean-field framework here for simplicity. As indicated in Fig 2, the superconductivity persists in a non-magnetic state even at $\lambda^{\prime}=1$ for $x>0.082$. In our present calculation, this small $x$ superconducting state has bond-centered charge order of period 2, but more complex-bond-centered chargeordered states are also possiblet 45 . We also expect that the superconductivity will co-exist with antiferromagnetism, but we did not undertake a complete solution of the mean-field equations within the antiferromagnetic phase because of the complexity of the calculation. As in the insulator, the onset of antiferromagnetim was determined by the point where the $t_{\alpha}$ boson had a vanishing gap in its spectrum.

We characterized the superconducting state by determining pairing amplitudes, $\Delta_{x, y}$, which characterize the pairing of the holes, $h_{1,2}$, in the $x$ and $y$ directions. These quantities are described more precisely in Appendices B and व. Their values are plotted as a function of the hole concentration, $x$, in Fig 3 . Note that $\Delta_{x}$ and $\Delta_{y}$ are roughly of the same magnitude: this means that the superconductivity is genuinely two-dimensional, and there is no regime in which a quasi-one-dimensional LutherEmery liquid-like behavior holds. Indeed, it is even possible for $\Delta_{x}$ to be larger than $\Delta_{y}$, which would make the superconductivity stronger in the $x$ direction rather than in the vertical "stripe" direction. This latter phenomenon appears to be similar in spirit to the ananlyses of Refs 66 68.

For small $x$, the pairing amplitudes are non-zero but quite small; moreover, the pairing amplitudes in the horizontal and vertical directions have the same sign in our 


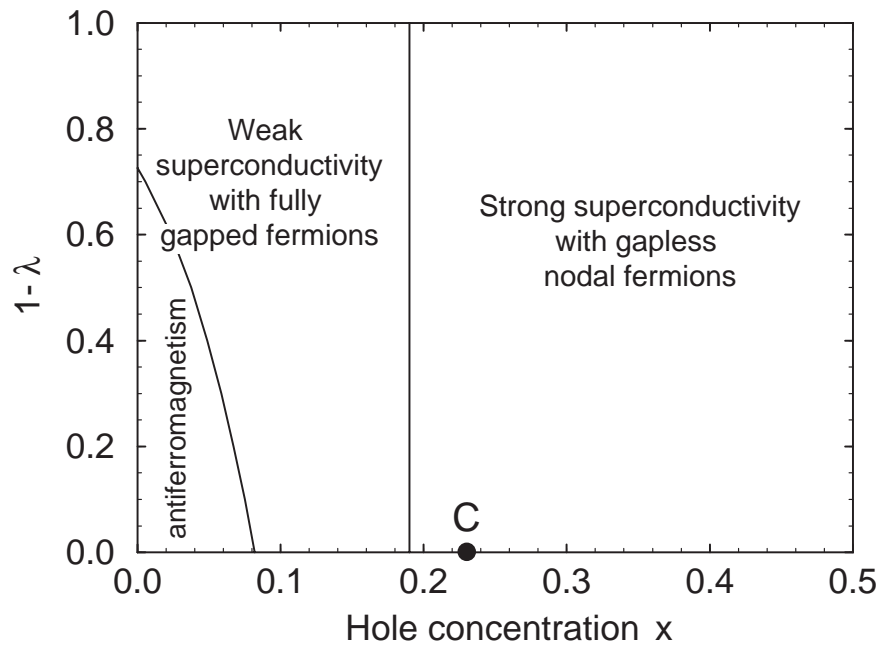

FIG. 2. Phase diagram of the square lattice Hamiltonian $H$ in (8) for the configuration shown in Fig $1 \mathrm{p}$. Results are shown here for $t^{\prime}=t, \lambda=1$ and $t / J=3.0$ as a function of the hole concentration $x$ and $1-\lambda^{\prime}$. The model has full square lattice symmetry at $1-\lambda^{\prime}=0$, and the exchange interaction separates into that on decoupled two-leg ladders at $1-\lambda^{\prime}=1$. The nearest-neighbor Coulomb repulsion, $V$ is taken to have a very large positive value, and the results are indistinguishable from those at $V=\infty$. The phase boundary towards antiferromagnetism was determined by the point at the $t_{\alpha}$ excitation spectrum had a vanishing spin gap, signaling the onset of a phase in which (10) was valid. The "weak" and "strong" superconductivity distinction is qualitative, and is indicated by the rapid change in the physical superconducting order parameter in Fig 3 around $x=0.2$. The antiferromagnetic phase co-exists with weak superconductivity at non-zero $x$. The nearly vertical phase boundary indicates the quantum phase transition at which nodal fermions first appear: this transition is discussed in Section IV. C denotes the point where the spontaneous bond-centered charge order of the square lattice is expected to disappear with increasing $x$; the bond-centered charge order is explicitly present in the Hamiltonian for $1-\lambda^{\prime}>0$, and is spontaneous only for non-zero $x$ before the point $\mathrm{C}$ and $1-\lambda^{\prime}=0$. The present bond operator approach was not used to determine the position of $\mathrm{C}$; nevertheless, following Ref 0 we are able to present a theory of the critical properties of $\mathrm{C}$ in Appendix $\mathrm{A}$

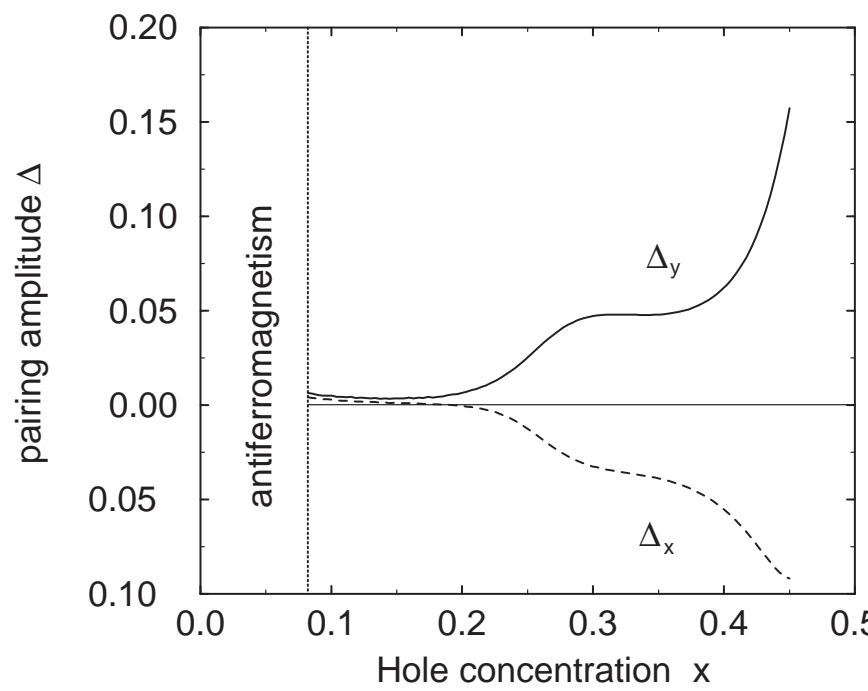

FIG. 3. Pairing amplitudes $(\Delta)$ as a function of hole concentration $(x)$ when $t / J=3.0$, and $\lambda=\lambda^{\prime}=1.0$, i.e. the isotropic case. The pairing amplitude in the vertical direction of Fig $1 \mathrm{~b}$ is $\Delta_{y}$, and that in the horizontal direction is $\Delta_{x}$. For $x \leq 0.082$, there is instability to long-range antiferromagnetism due to the collapse of the gap of $S=1$ particle excitation, $t_{\alpha}$. When $0.082<x \leq 0.192$, non-magnetic superconducting state is stable, and the pairing amplitudes are of the same sign, but of very small value. For doping concentration larger than roughly 0.2 , the pairing amplitudes have opposite signs, which, in turn, gives rise to nodal fermions.

mean field theory. We will refer to this as the weak superconductivity regime. It is possible that, upon including quantum fluctuations, the ground state in this weak pairing regime is easily susceptible to a quantum transition to an insulating state with some additional translational symmetry breaking. Also note that the pairing amplitude is nonzero at the boundary of the onset of antiferromagnetic order: we therefore expect the superconductivity to survive within the antiferromagnetic phase.

For larger $x$, the pairing amplitude in Fig 3 increases rapidly and we reach a "strong superconductivity" regime. The pairing amplitudes now have opposite signs in the horizontal and vertical directions, and this permits gapless nodal fermionic quasiparticle excitations, as shown explicitly in our results below. However, there is one important feature associated with the appearance of the nodal particles that is worth emphasizing. Originally, our calculations were carried out with the nearestneighbor Coulomb repulsion $V=0$. In this case we found that the pairing of the holes occurred primarily by the condensation of the $d$ bosons. Because of the resulting very short-range pairing we found no nodal points, even though the pairing amplitude had opposite signs in the horizontal and vertical directions. It was only when we had turned on a $V$ of the order of the bandwidth, which significantly reduced the amplitude of the $d$ boson condensate and made the hole pairing more long-ranged, did we find the appearance of the nodal excitations. 


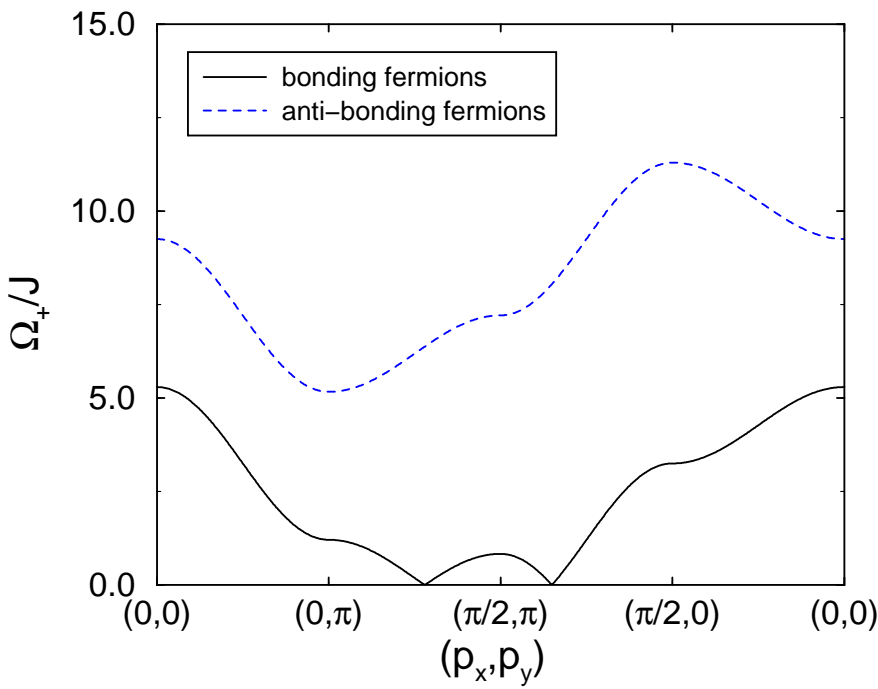

FIG. 4. Dispersion curves of the bonding and anti-bonding $=1 / 2$ fermionic excitations in the weak pairing regime with $x=0.1, t / J=3.0$, and $\lambda=\lambda^{\prime}=t^{\prime} / t=1$. There appear to be fermionic excitations at energies very close to 0 , but the blow-up in Fig $\mathrm{A}$ shows that there is indeed a gap in the fermionic spectrum. The $x$-axis is chosen to take a representative straight path connecting points in the first Brillouin zone: from $\left(p_{x}, p_{y}\right)=(0,0)$ to $(0, \pi)$ to $(\pi / 2, \pi)$ to $(\pi / 2,0)$ to $(0,0)$.

We now describe our results for the elementary excitations of the superconducting states in our phase diagram. First, we discuss the fermionic $S=1 / 2$ excitations; in principle, these are observable in photoemission or tunneling experiments. Note that the unit cell of Fig $\mathbb{1} b$ has two sites, and so the first Brillouin zone extends between $-\pi / 2$ and $\pi / 2$ in the $x$ direction, and between $-\pi$ and $\pi$ (as usual) in the $y$ direction. Consequently, half the fermionic excitations in the first Brillouin zone of the original square lattice will be folded back, by a Bragg reflection in the vertical planes at $\pm \pi / 2$, into a second band in the first Brillouin zone of the lattice of Fig $1 \mathrm{~b}$. We will refer to these two bands as the 'bonding' and 'antibonding' bands, based upon their wavefunctions within the dimers, as discussed in Appendix B. We show the $S=1 / 2$ fermionic bands in the weak-pairing regime in Figs 4 and 5. There is a gap across the entire Brillouin zone. However, because of the very small pairing amplitude, the fermionic excitation energy becomes very small along an incipient "Fermi surface" in the Brillouin zone. This Fermi surface line is indicated by the solid line in the inset of Fig 5. The position of this Fermi surface is similar to that in the computation by Sushkov41, who also discussed its relationship to photoemission experiments.

Next, we turn to a discussion of the fermionic excitations at larger $x$, where the superconductivity is stronger. The $S=1 / 2$ excitation spectra are now shown in Figs 6 and 7. Overall, the results at higher energies, are quite similar to those in Figs 4 and 5 , but there is now a dramatic difference at lower energies. There is a gapless

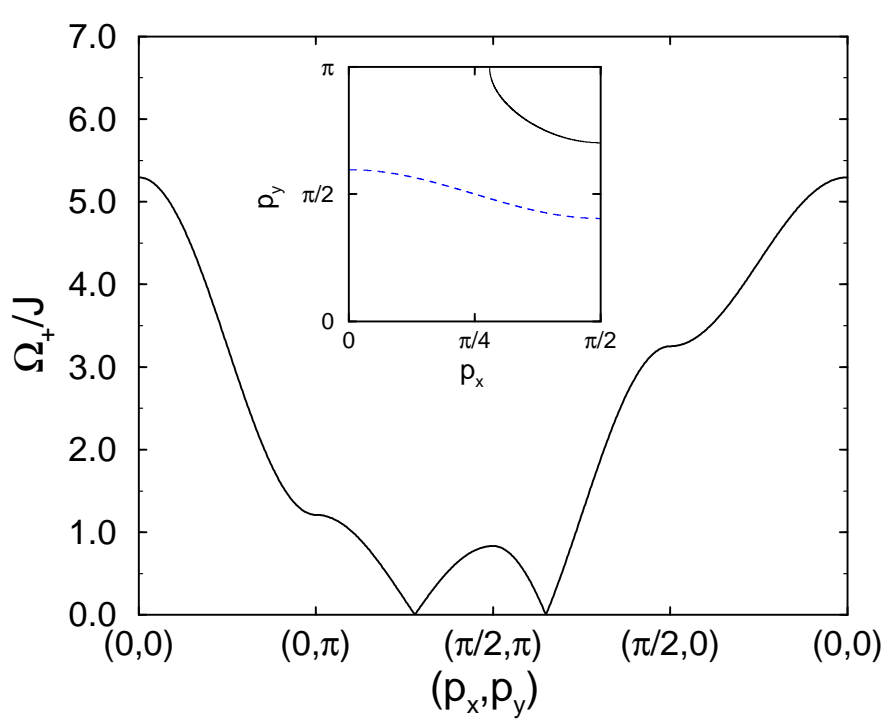

FIG. 5. Blow-up of the fermionic dispersion in Fig 4 at the lowest non-zero energies. Notice that there is indeed a finite gap in the fermionic spectrum. This is also made clear in the inset: the solid line shows the line where the fermion kinetic energy vanishes (the incipient Fermi surface) while the dashed line indicates where the fermion pairing amplitude vanishes. For gapless excitations, both quantities have to vanish, and the absence of a crossing point between the lines indicates that a gap is always present. The solid line in the inset is also the location of the incipient Fermi surface which has been quenched by pairing - the position of this surface is similar to that in Ref 41 .

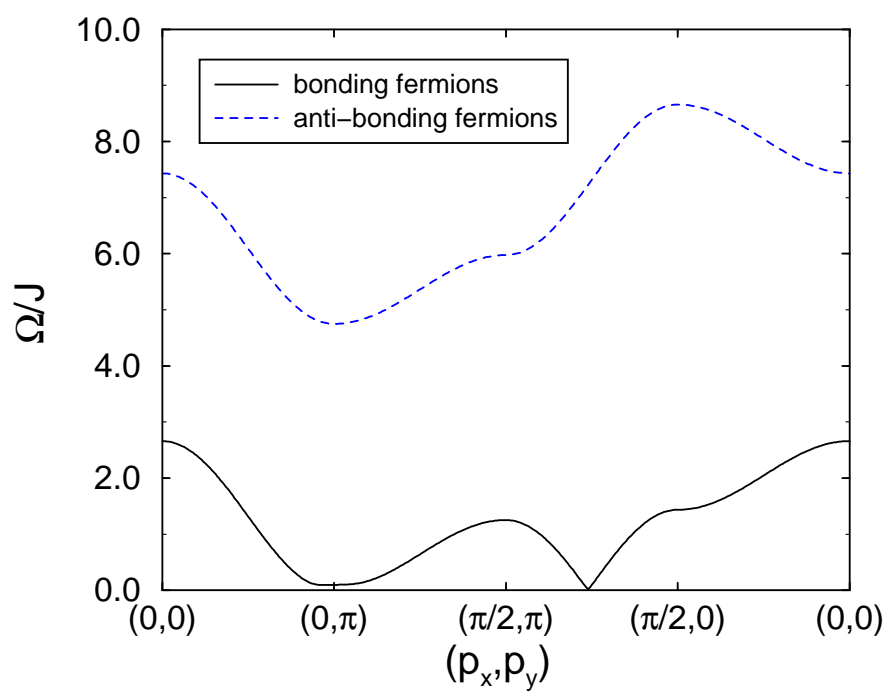

FIG. 6. Dispersion curves of the bonding and anti-bonding $S=1 / 2$ fermionic excitations with $x=0.3, t / J=3.0$, and $\lambda=\lambda^{\prime}=t^{\prime} / t=1$ (as in Fig $⿴$ but at a larger doping). At this value of $x$, there is a nodal quasiparticle excitation not too far from $(\pi / 2, \pi / 2)$. 


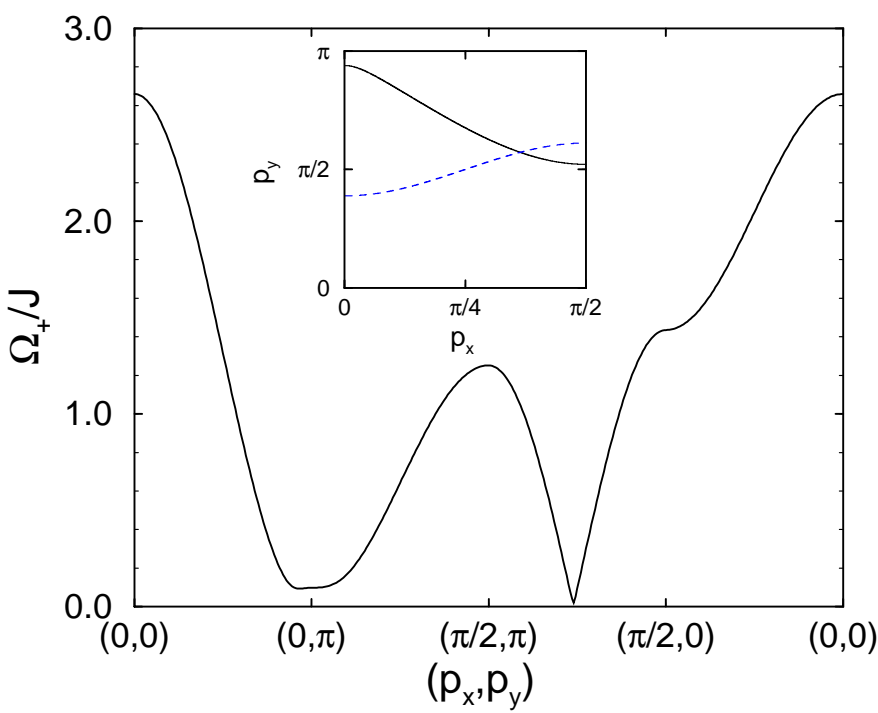

FIG. 7. Blow-up of the fermionic dispersion in Fig at the lowest non-zero energies (as in Fig 5 but at a larger doping). Notice that there is clearly a gapless nodal point not too far from $(\pi / 2, \pi / 2)$, as is also clear from the inset, where the two curves now cross. The pairing gap near the "antinodal point", $(\pi, 0)$, remains finite.

nodal-point near $(\pi / 2, \pi / 2)$, and this is made possible by the $d$-wave-like pairing amplitudes. The spectra in Fig 5 and 7 cannot be continuously connected, and there must be a singular phase transition point at which the nodal fermion first appears. The position of this phase boundary is indicated in Fig 2, and the nature of the phase transition will be discussed below in Section IV.

Finally, we consider the $S=1$ excitations, which are visible in neutron scattering experiments, There are two categories of such excitations. First, we have the $S=1$, $t_{\alpha}$ particles, with a definite energy-momentum relation: these may be viewed as a collective spin mode (or a triplet exciton) which goes soft at the antiferromagnetic ordering transition. These are the only $S=1$ excitations in the undoped antiferromagnet, but, connect smoothly to corresponding excitations in the doped antiferromagnet. The second class of $S=1$ excitations are scattering states of the fermionic $S=1 / 2$ excitations just discussed. These do not have a definite energy momentum relation, but exist over a continuum in a range of energies at any given momentum. We show a plot of the spectra of these two $S=1$ excitations in Figs 8 and 9 .

At low doping, in Fig 8, not that the $t_{\alpha}$ excitation has a well-formed minimum at $(0, \pi)$, and this minimum is below the lower-bound of the two-particle continuum. So the $t_{\alpha}$ excitations are absolutely stable towards decay into 2 fermionic $S=1 / 2$ excitations over this range of energy and momenta. This stable $S=1$ excitonic excitation will lead to a resonance peak (in principle, infinitely sharp) in the neutron scattering cross-section at the energy and momentum of the $t_{\alpha}$ particle. Also, as the doping is lowered, the minimum of the $t_{\alpha}$ disper-

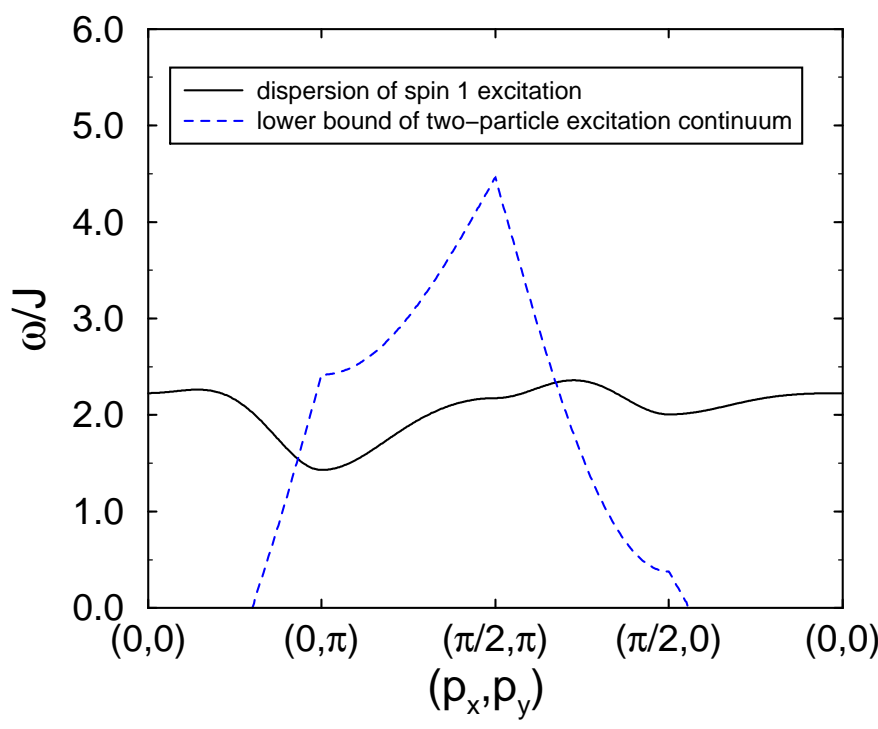

FIG. 8. Dispersion curve (full line) of $S=1, t_{\alpha}$ particle excitation with $x=0.1, t / J=3.0$, and $\lambda=\lambda^{\prime}=t^{\prime} / t=1$. The pairing is weak for this value of doping. Also shown (dashed line) is the lower bound of the two-particle continuum made up of a pair of $S=1 / 2$ excitations from Fig 4 and 6 . Note that the minimum of the $t_{\alpha}$ excitation is at $(0, \pi)$, but because of the halving of the Brillouin zone this wavevector is crystalographically equivalent to $(\pi, \pi)$.

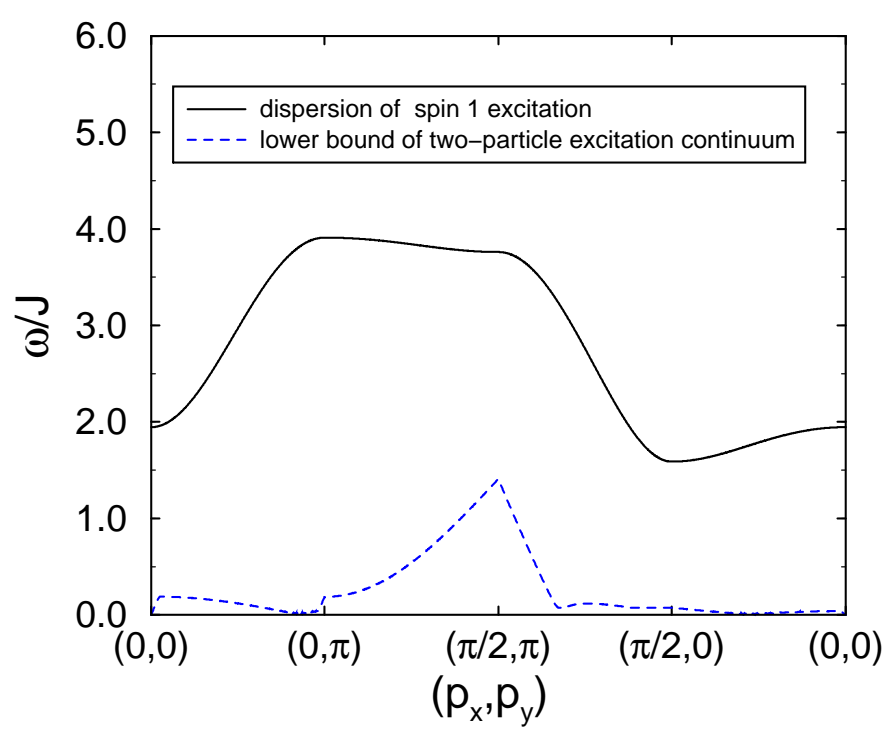

FIG. 9. As in Fig 8, but in the strong pairing region with nodal quasiparticles at $x=0.3$. 
sion gets lowered, until it hits zero also in a region which has no overlap with the fermionic two-particle continuum. This signals the transition to the antiferromagnetic phase: the absence of low energy fermionic excitations at near the minimum $t_{\alpha}$ energy implies that the critical theory for the transition remains identical to that for the corresponding transition in the insulator, which has been discussed in much detaillt4 The condensation of the $t_{\alpha}$ bosons occurs at momentum $(0, \pi)$, but note that because of the doubling of the unit cell this momentum is equivalent to $(\pi, \pi)$. Determination of the microscopic configuration of the spins using the operator representation in Section II shows that the mean moments indeed have the staggered arrangement associated with the conventional, two-sublattice Néel state.

The higher doping $S=1$ excitations are sketched in Fig 9. Now the $t_{\alpha}$ excitations are well within the twofermionic-particle continuum, and so will quickly decay and lose their identity. Note that the doping at which the $t_{\alpha}$ excitations ceases to be stable at any momentum does not (in general) co-incide with the point at which nodal fermionic quasiparticles appear. As long as the nodal points are not exactly at $(\pi / 2, \pi / 2)$, the $t_{\alpha}$ excitations can be stable at very low energies near $(\pi, \pi)$. In the present computation, the nodal points are quite close to $(\pi / 2, \pi / 2)$ and to the $t_{\alpha}$ excitation is quickly quenched. In a more general model, with second neighbor hopping, the $t_{\alpha}$ excitations could be stable even in an isotropic $d$-wave superconductor, provided we moved the nodal points sufficiently far from $(\pi / 2, \pi / 2)$.

\section{ONSET OF GAPLESS NODAL FERMIONIC EXCITATIONS}

The phase diagram of Fig 2 shows a phase boundary demarking superconductors with and without nodal quasiparticles. This is a quantum phase transition in the sense that there is a (weak) non-analyticity in the ground state energy as a function of doping at this point. We will present a simplified treatment which captures its essential universal features. Such a transition was discussed earlier in Ref 7 and by Granath et al. 46 ; the latter authors also reached conclusions on the universal properties which agree with our discussion here. A related, but distinct, theory for the annihilation of nodal particles in a $d$-wave superconductor was also discussed by Duncan and Sá de Melo47. They considered an isotropic superconductor as a function of electron density, and found that the nodal points vanished when all four of them collided at $k=0$. This transition is differs from that in the anisotropic superconductors of interest here, where the nodal points collide only in pairs, and the quantum critical points belong to distinct universality classes.

We approach this transition from the side of the $d$ wave superconductor with full square lattice symmetry. Here, the fermionic excitations are described by the BCS Hamiltonain

$$
H_{B C S}=\sum_{k}\left[\epsilon(k) c_{k a}^{\dagger} c_{k a}+\frac{\Delta(k)}{2}\left(\varepsilon_{a b} c_{k a}^{\dagger} c_{-k b}^{\dagger}+\text { H. c. }\right)\right],
$$

where, in the simplest nearest-neighbor model $\epsilon_{k}=$ $-2 t\left(\cos k_{x}+\cos k_{y}\right)-\mu$, and the pairing energy $\Delta(k)=$ $\Delta_{0}\left(\cos k_{x}-\cos k_{y}\right)$. We now assume that there is an onset of bond-centered charge order at wavevector $G=(\pi / a, 0)$ of amplitude $\psi_{\mathrm{sp}}$-because this charge order wave is in the $x$ direction, $\psi_{\mathrm{sp}}$ is the real part of the more general order parameter, $\Psi_{\mathrm{sp}}$ considered in Section [1] and Appendix A. This order will lead to a modulation in the fermion hopping matrix element and the pairing interaction at the wavevector $G$, and so induce the following additional terms in the Hamiltonian for the fermionic excitations

$$
\begin{aligned}
H_{\mathrm{sp}}=i \psi_{\mathrm{sp}} \sum_{k}\left[a(k) c_{k, a}^{\dagger} c_{k+G, a}\right. & \\
& \left.\quad+\frac{b(k)}{2}\left(\varepsilon_{a b} c_{k a}^{\dagger} c_{-k+G, b}^{\dagger}+\text { H. c. }\right)\right],
\end{aligned}
$$

where $a(k)=w_{1} \sin k_{x}$ and $b(k)=w_{2} \sin k_{x}$, with $w_{1,2}$ some constants. These last factors of $\sin k_{x}$ are a conse quence of the bond-centered nature of the charge orden 48 , but the results of this section are not crucially dependent upon this fact; similar results will apply also to site-centered charge orders.

It is possible to diagonalize the Hamitonian $H_{B C S}+$ $H_{\text {sp }}$ and determine the fermionic excitation spectrum of the state with co-existing superconductivity and charge order. The energy eigenvalues are

$$
\left[\Lambda_{1} \pm\left(\Lambda_{1}^{2}-\Lambda_{2}^{2}-\Lambda_{3}^{2}\right)^{1 / 2}\right]^{1 / 2}
$$

where

$$
\begin{gathered}
\Lambda_{1} \equiv\left[\epsilon^{2}(k)+\epsilon^{2}(k+G)+\Delta^{2}(k)+\Delta^{2}(k+G)\right] / 2 \\
+\psi_{\mathrm{sp}}^{2}\left[a^{2}(k)+b^{2}(k)\right] \\
\Lambda_{2} \equiv \Delta(k) \epsilon(k+G)+\Delta(k+G) \epsilon(k)-2 \psi_{\mathrm{sp}}^{2} a(k) b(k) \\
\Lambda_{3} \equiv \psi_{\mathrm{sp}}^{2}\left[a^{2}(k)-b^{2}(k)\right] \\
+\Delta(k) \Delta(k+G)-\epsilon(k) \epsilon(k+G) .
\end{gathered}
$$

It is instructive to examine the evolution of the zeros of (14) as a function of $\psi_{\mathrm{sp}}$. For $\psi_{\mathrm{sp}}=0$, there are four symmetric nodal points determined by the solutions of $\epsilon(k)=0$ and $\Delta(k)=0$. It is assumed (as is the case at non-zero doping and in the absence of particle-hole symmetry), that the wavevector separating any two of these nodal points is not equal to $G$. If it was equal to $G$, then the nodal points would be gapped at an infinitesimal value of $\psi_{\text {sp }}$, and a non-trivial theory would describe the quantum critical fluctuations, as has already been discussed in Ref 7 . Turning to the more general situation, where the separation between the nodal points is 
not equal to $G$, the quantum critical theory describing the onset of a non-zero $\psi_{\text {sp }}$ is reviewed in Appendix the leading critical singularities do not involve the nodal fermions. As $\psi_{\text {sp }}$ increases, examination of (14) shows that the four nodal points move towards the new Brillouin zone boundary at $k_{x}= \pm \pi / 2$. Eventually, these points collide in pairs at

$$
p_{0}=\left(\pi / 2, p_{0 x}\right)
$$

(and symmetry related points) and $\psi_{\mathrm{sp}}=\psi_{\mathrm{sp}}^{c}$, and there are no nodal points for $\psi_{\mathrm{sp}}>\psi_{\mathrm{sp}}^{c}$. We are interested here in describing the quantum critical theory of this nodal point collision. Clearly, the fluctuations of $\psi_{\mathrm{sp}}$ will not be critical at this point, as the transition occurs at a non-zero value of $\psi_{\mathrm{sp}}^{c}$.

First, let us determine the values of $\psi_{\mathrm{sp}}^{c}$ and $p_{0}$. Using the facts that $\epsilon\left(p_{0}\right)=\epsilon\left(p_{0}+G\right)$ and $\Delta\left(p_{0}\right)=\Delta\left(p_{0}+G\right)$, the condition for the presence of gapless nodal points in (14) becomes

$$
\epsilon\left(p_{0}\right)=\psi_{\mathrm{sp}}^{c} a\left(p_{0}\right) \quad ; \quad \Delta\left(p_{0}\right)=\psi_{\mathrm{sp}}^{c} b\left(p_{0}\right) .
$$

The solution of these two equations determines the two unknowns $\psi_{\mathrm{sp}}^{c}$ and $p_{0 x}$. At this same point, the second eigenvalue in (14) remains non-zero and finite. This fermionic mode will play no role in the critical theory, and so it pays to perform a canonical transformation at an early stage to project it out. To leading order in the distance from the critical point, this merely means that we have to take only the linear combination of $c_{k a}$ and $c_{k+G, a}$ which appears in gapless eigenvalue associated with (16). So, we introduce a new fermionic degree of freedom, $f_{q a}^{+}$, where the small momentum $q$ is measured as a deviation from $p_{0}$ (and a corresponding fermionic mode $f_{q a}^{-}$which resides at momenta near $\left.-p_{0}\right)$; using the structure of the gapless eigenvalue at $\psi_{\mathrm{sp}}=\psi_{\mathrm{sp}}^{c}$ and $k=p_{0}$, we see that we should parameterize

$$
\begin{aligned}
c_{k a} & =f_{q a}^{+} / \sqrt{2} \\
c_{k+G, a} & =i \varepsilon_{a b} f_{q b}^{+} / \sqrt{2},
\end{aligned}
$$

where $k=p_{0}+q$, and $q$ is small; a similar parameterization is made near $-p_{0}$ with $f_{a}^{-}$. Finally, we insert (17) into $H_{B C S}+H_{\mathrm{sp}}$, and expand in $\psi_{\mathrm{sp}}-\psi_{\mathrm{sp}}^{c}$ and in gradients of $f_{a}^{ \pm}$. This leads to the following effective action for the critical theory

$$
\begin{aligned}
S_{f} & =\int d^{2} r d \tau\left[f_{a}^{ \pm \dagger}\left(\frac{\partial}{\partial \tau} \pm i v_{1} \frac{\partial}{\partial y}-\frac{1}{2 m_{1}} \frac{\partial^{2}}{\partial x^{2}}+\delta_{1}\right) f_{a}^{ \pm}\right. \\
& +\varepsilon_{a b} f_{a}^{-}\left(v_{2} \frac{\partial}{\partial y}-\frac{1}{2 m_{2}} \frac{\partial^{2}}{\partial x^{2}}+\delta_{2}\right) f_{b}^{+}+\text {H.c. }
\end{aligned}
$$

where $\tau$ is imaginary time, $r=(x, y)$ is the spatial coordinate, $\delta_{1,2}=w_{1,2}^{\prime}\left(\psi_{\mathrm{sp}}-\psi_{\mathrm{sp}}^{c}\right)$, and $v_{1,2}, m_{1,2}, w_{1,2}^{\prime}$ are constants dependent upon the detailed momentum dependence of $\epsilon(k)$ and $\Delta(k)$. The fermionic eigenenergy of (18) is easily determined; it is

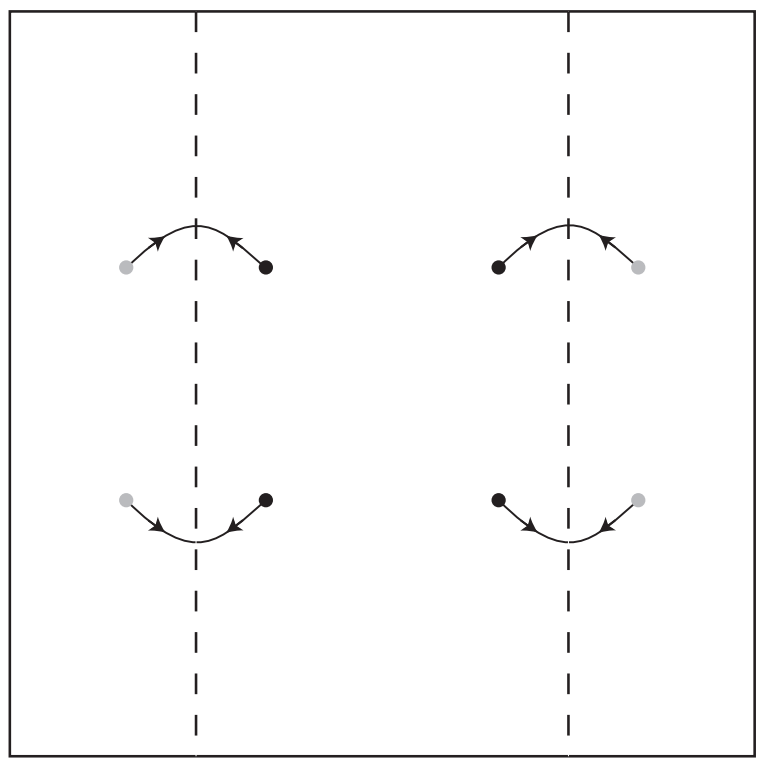

FIG. 10. Evolution of the gapless nodal points in the fermionic excitation spectrum as a function of $\psi_{\mathrm{sp}}$. The square contains the first Brillouin zone of the original square lattice, extending between $k_{x, y}= \pm \pi$. The dark circles are the positions of the nodal points in this $d$-wave superconducvtor. Onset of a non-zero $\psi_{\text {sp }}$ introduces Bragg reflection planes at $k_{x}= \pm \pi / 2$ and images of the nodal points indicated by the grey circles. As $\psi_{\mathrm{sp}}$ increases, the nodal points move towards the Bragg-reflection planes and annihilate each other when they collide The critical theory of this transition is discussed in Section IV.

$$
\left[\left(\delta_{1}+v_{1} q_{y}+\frac{q_{x}^{2}}{2 m_{1}}\right)^{2}+\left(\delta_{2}+v_{2} q_{y}+\frac{q_{x}^{2}}{2 m_{2}}\right)^{2}\right]^{1 / 2}
$$

The positions of the nodal points, if present, are easily determined from (19). Assuming $v_{1,2}, m_{1,2}, w_{1,2}^{\prime}$ are all positive, then for $w_{1}^{\prime} / v_{1}>w_{2}^{\prime} / v_{2}$ and $m_{1} v_{1}<m_{2} v_{2}$, nodal points are present for $\psi_{\mathrm{sp}}<\psi_{\mathrm{sp}}^{c}$, but not for $\psi_{\mathrm{sp}}>$ $\psi_{\mathrm{sp}}^{c}$ (similar results hold for other signs and magnitudes of the various coupling constants). The trajectory of the nodal points is sketched schematically in Fig 10: they move on a parabolic trajectory with $\left|q_{y}\right| \sim q_{x}^{2}$ before colliding along the Bragg reflection planes at $k_{x}= \pm \pi / 2$.

The availability of the action $S_{f}$ also allows one to determine the consequences of the interactions near the quantum critical point. Notice that at the critical point, $\delta_{1}=\delta_{2}=0, S_{f}$ is invariant under the scale transformation $\tau \rightarrow \tau / s, y \rightarrow y / s, x \rightarrow x / \sqrt{s}$, and $f \rightarrow s^{3 / 4} f$. The simplest allowed four fermion coupling is $\sim\left(f^{\dagger} f\right)^{4}$, and power-counting shows that its co-efficient has a negative scaling dimension of $-1 / 2$. So this interaction is irrelevant perturbation at the quantum critical point 16 . In a 
similar manner, it is not difficult to show that all other perturbations of $S_{f}$ are irrelevant, and so $S_{f}$ is the complete critical theory of the transition involving the onset of nodal fermionic excitations.

\section{COUPLING OF SPIN PEIERLS ORDER TO PHONONS}

A central actor in all the considerations so far has been the spin-Peierls order parameter, $\Psi_{\mathrm{sp}}$. Experimental observations of the fluctuations of this order parameter would certainly be helpful in resolving the theoretical issues. However, this is a spin-singlet, charge zero mode and is only observable through its couplings to the ionic displacements. This section will therefore present a simplified discussion of the coupling between the dynamics of the spin Peierls order parameter and the phonon modes of the $\mathrm{CuO}_{2}$ plane. Indeed, it is probable that the phonons are more than merely spectators of the spin dynamics, and the spin-phonon coupling may play an important role in selecting between different charge orderings and in influencing the nature of the electronic ground state: the important phonon and spin excitation energies are roughly of the same order, and so a coupled dynamical model will be necessary for a complete microscopic understanding.

The spin-Peierls order parameter couples most directly to the "Peierls-active" phonon mode sketched as mode $\mathrm{C}$ in Fig 11: this is a staggered displacement of the $\mathrm{Cu}$ ions at the wavevector $(\pi, 0)$. Notice that the $\mathrm{O}$ ions are stationary in this phonon mode. As we will describe below, the coupling between this Peierls-active phonon and the spin-Peierls order parameter is likely to lead to an additional low energy peak in the dynamic phonon structure factor at the energy scale of the characteristic spin-Peierls fluctuations. However, neutron scattering studies of the phonon spectra have so far 21.4951 focused on optical phonons involving motion primarily of the $\mathrm{O}$ ions (the mode between A and B in Fig 11): the smaller $\mathrm{O}$ ion mass, $M_{\mathrm{O}} \approx M_{\mathrm{Cu}} / 4$, increases the displacement of the $\mathrm{O}$ ions and makes these easier to observe. The $\mathrm{O}$ ion phonon at wavevector $(\pi, 0)$ (mode B in Fig 11) is not Peierls-active in the sense just noted. However, following the suggestion of McQueeney et al.21, we will describe below a non-linear coupling in the spin-phonon Hamiltonian which leads to signatures of the spin-Peierls fluctuations in the A-B phonon mode at the wavevector $(\pi / 2,0)$.

It is also worth mentioning the recent work of Khaliullin and Horsch 22 . In contrast to our focus on bondcentered charge fluctuations, they considered $\mathrm{Cu}$ sitecentered charge density fluctuations at wavevectors near $(\pi, 0)$ (in the notation of Nayak 48 , we are examining $p_{x}$ density wave correlations at $(\pi, 0)$, while Ref 52 discusses $s$ density wave correlations at $(\pi, 0))$. These $\mathrm{Cu}$ sitecentered fluctuations do indeed couple linearly to the

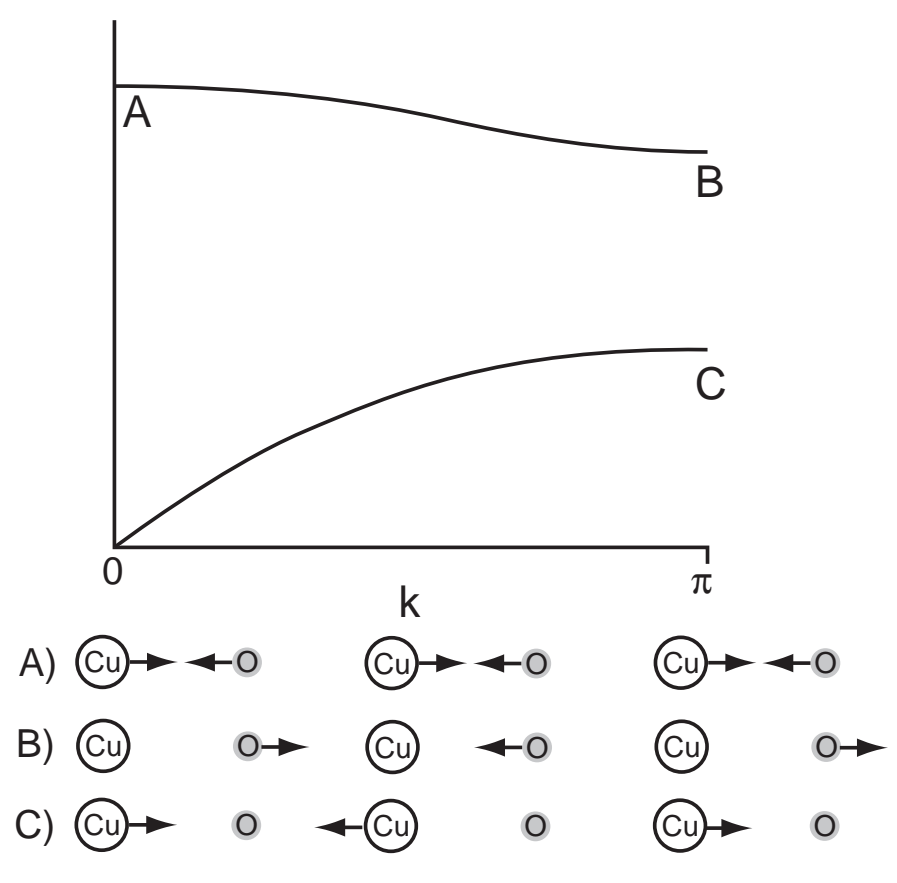

FIG. 11. Phonon frequencies (vertical axis) of the one-dimensional $\mathrm{Cu}-\mathrm{O}$ chain. The frequency of the mode $\mathrm{A}$ is $\left[2 K\left(M_{\mathrm{Cu}}+M_{\mathrm{O}}\right) /\left(M_{\mathrm{Cu}} M_{\mathrm{O}}\right)\right]^{1 / 2}$, that of $\mathrm{B}$ is $\left[2 K / M_{\mathrm{O}}\right]^{1 / 2}$, and that of $\mathrm{C}$ is $\left[2 K / M_{\mathrm{Cu}}\right]^{1 / 2}$. Only mode $\mathrm{C}$ is "Peierls active" i.e. couples linearly to the order parameter $\Psi_{\mathrm{sp}}$, which measures the amplitude of a bond-centered charge order wave at wavevector $(\pi / a, 0)$. 
phonon mode in the vicinity of the point B in Fig 11: this led to a broadening and softening of the phonon near B. As we will see below, our bond-centered spin-Peierls correlations instead modify the A-B phonon mode near $(\pi / 2,0)$ in a manner which is consistent with experimental observations.

We will work here with a simplified one-dimensional model of the phonons in the $\mathrm{CuO}_{2}$ plane. All the phonons we are interested in are polarized both in wavevector and ionic displacement along the $(1,0)$ axis, and so neglecting the second dimension is not too serious. We will consider only a single chain of alternating $\mathrm{Cu}$ and $\mathrm{O}$ ions, and consider displacements of the ions along the chain direction (see Fig 11). A more complete treatment is certainly possible, but we will not attempt it here: we hope that with improved experimental resolution, a more precise and microscopic theoretical study of the two-dimensional phonon modes will be carried out, along the lines of the analyses of Refs 53,54 for $\mathrm{CuGeO}_{3}$.

We begin by specifying our toy one-dimensional model of the phonons in an alternating chain of $\mathrm{Cu}$ and $\mathrm{O}$ ions. We place the $\mathrm{Cu}$ ions on the sites, $i$ (integer), of a chain with spacing $a$, while the $\mathrm{O}$ ions are at the centers of the links of the chain. We denote the displacement of these ions by $u_{i}$ and $v_{i}$ respectively, where the $i$ th $\mathrm{O}$ ion is taken to be to immediately to the right of the $i$ 'th $\mathrm{Cu}$ ion. The harmonic action for the phonon modes is (in imaginary time, $\tau$ ):

$$
\begin{aligned}
S_{\mathrm{ph}} & =\int d \tau \sum_{i}\left[\frac{M_{\mathrm{Cu}}}{2}\left(\frac{d u_{i}}{d \tau}\right)^{2}+\frac{M_{\mathrm{O}}}{2}\left(\frac{d v_{i}}{d \tau}\right)^{2}\right. \\
& \left.+\frac{K}{2}\left\{\left(u_{i}-v_{i}\right)^{2}+\left(u_{i}-v_{i-1}\right)^{2}\right\}\right],
\end{aligned}
$$

where $K$ is a "spring constant" which determines the phonon frequencies. It is a simple matter to diagonalize $S_{\mathrm{ph}}$ and obtain the phonon normal mode frequencies: they are

$$
\begin{gathered}
\omega_{ \pm}^{2}=\frac{K}{M_{\mathrm{Cu}} M_{\mathrm{O}}}\left[M_{\mathrm{Cu}}+M_{\mathrm{O}} \pm\left(\left(M_{\mathrm{Cu}}-M_{\mathrm{O}}\right)^{2}\right.\right. \\
\left.\left.+4 M_{\mathrm{Cu}} M_{\mathrm{O}} \cos ^{2}(k / 2)\right)^{1 / 2}\right]
\end{gathered}
$$

and are sketched in Fig 11 .

We also have to consider the dynamics of the spin Peierls order $\Psi_{\mathrm{sp}}$ introduced in Section and considered in Section IV and Appendix A. In our present one-dimensional toy model, we need only consider $\psi_{\mathrm{sp}}=$ $\operatorname{Re}\left[\Psi_{\mathrm{sp}}\right]$. For this order parameter $\psi_{\mathrm{sp}}$, the dynamics of an interacting effective action like $S$ in (A1) is assumed to be captured by the following effective quadratic actiont:

$$
\begin{aligned}
S_{\mathrm{sp}} & =\frac{T}{2} \sum_{k, \omega_{n}}\left|\psi_{\mathrm{sp}}\left(k, \omega_{n}\right)\right|^{2} \chi_{\mathrm{sp}}^{-1}\left(k, i \omega_{n}\right) \\
\chi_{\mathrm{sp}}^{-1}\left(k, i \omega_{n}\right) & \equiv \omega_{n}^{2}+2 a^{2} c_{1}^{2}(1-\cos (k))+\Delta_{\mathrm{sp}}^{2}+\Gamma_{\mathrm{sp}}\left|\omega_{n}\right| .
\end{aligned}
$$

Here $\omega_{n}$ is a Matsubara frequency, and $\Delta_{\mathrm{sp}}$ and $\Gamma_{\mathrm{sp}}$ are effective energy scales determining the mean frequency and damping of the $\psi_{\mathrm{sp}}$ fluctuations; the values of $\Delta_{\mathrm{sp}}$ and $\Gamma_{\mathrm{sp}}$ are determined by the non-linear interactions in (A1). Also we have replaced the spatial gradient in (A1) by a nearest-neighbor lattice derivative in (22). As one approaches the onset of spin-Peierls order (approaching point $\mathrm{C}$ in Fig 2 from the right), the value of $\Delta_{\mathrm{sp}}$ will decrease to zero, while $\Gamma_{\mathrm{sp}}$ becomes of order $T$, representing the damping of the order parameter mode in the quantum-critical region. We will work here in the $\Delta_{\text {sp }}>0$ regime, staying to the right of $\mathrm{C}$ in Fig 2. The velocity $c_{1}$ should be order the spin-wave velocity, and this is about 10 times larger than the velocity of the acoustic phonon mode, C, in Fig 11 .

Finally, we have to couple $\psi_{\mathrm{sp}}$ to the phonon modes. This coupling 5356 arises from the dependence of the exchange constant $J$ between neighbor $\mathrm{Cu}$ spins on the displacements of the $\mathrm{Cu}$ and $\mathrm{O}$ ions. We assume that $J \sim t_{p d}^{4}$, where $t_{p d}$, the overlap between neighboring $\mathrm{O}$ and $\mathrm{Cu}$ orbitals is a function of $\left(u_{i}-v_{i}\right)$ and $\left(u_{i}-v_{i-1}\right)$. Expanding $J$ in derivatives of these variables, we obtain first the simple linear coupling

$$
S_{1 c}=\int d \tau \sum_{i}\left[\lambda(-1)^{i} \psi_{\mathrm{sp}, i}\left(u_{i+1}-u_{i}\right)\right],
$$

where $\lambda$ is the linear coupling constant, and $\psi_{\mathrm{sp}, i}$ is the spin-Peierls order parameter in real space; this naturally resides on the centers of the bonds, and we locate $\psi_{\mathrm{sp}, i}$ on the same $\mathrm{O}$ site as $v_{i}$. As we noted earlier, $S_{1 c}$ couples the spin-Peierls order most strongly to the phonons in the vicinity of the point $\mathrm{C}$ in Fig 11, and will lead to its broadening and softening. We are interested here primarily in the modifications of the optical phonon mode A-B, and so we will neglect $S_{1 c}$ in our computations below.

As indicated at the beginning of this section, the important effect on the A-B optical phonon arises from a non-linear spin-phonon coupling . Expanding to one higher order in the phonon displacements, the coupling between $\psi_{\mathrm{sp}}$ and the phonon displacements can be written in the form

$$
\begin{aligned}
S_{2 c} & =\int d \tau \sum_{i}\left[( - 1 ) ^ { i } \psi _ { \mathrm { sp } , i } \left\{\gamma_{1}\left(u_{i+1}-u_{i}\right)^{2}\right.\right. \\
& \left.\left.+\gamma_{2}\left(u_{i+1}+u_{i}-2 v_{i}\right)^{2}\right\}\right],
\end{aligned}
$$

where $\gamma_{1,2}$ are the non-linear spin phonon coupling constants.

We examined the properties of $S_{\mathrm{ph}}+S_{\mathrm{sp}}+S_{2 c}$ in a simple one-loop approximation: we neglect the $\omega_{-}$phonon in (21), computed the self-energy of the $\omega_{+}$optical phonon at order $\gamma_{1,2}^{2}$. Finally, to compare to neutron scattering experiments, we computed

$$
D(k, \omega)=\left\langle\left|b_{\mathrm{Cu}} u(k, \omega)+b_{\mathrm{O}} v(k, \omega) e^{i k / 2}\right|^{2}\right\rangle,
$$

where $b_{\mathrm{Cu}}=7.718$ and $b_{\mathrm{O}}=5.803$ are the neutron scattering lengths of $\mathrm{Cu}$ and $\mathrm{O}$ respectively 5 . The results 

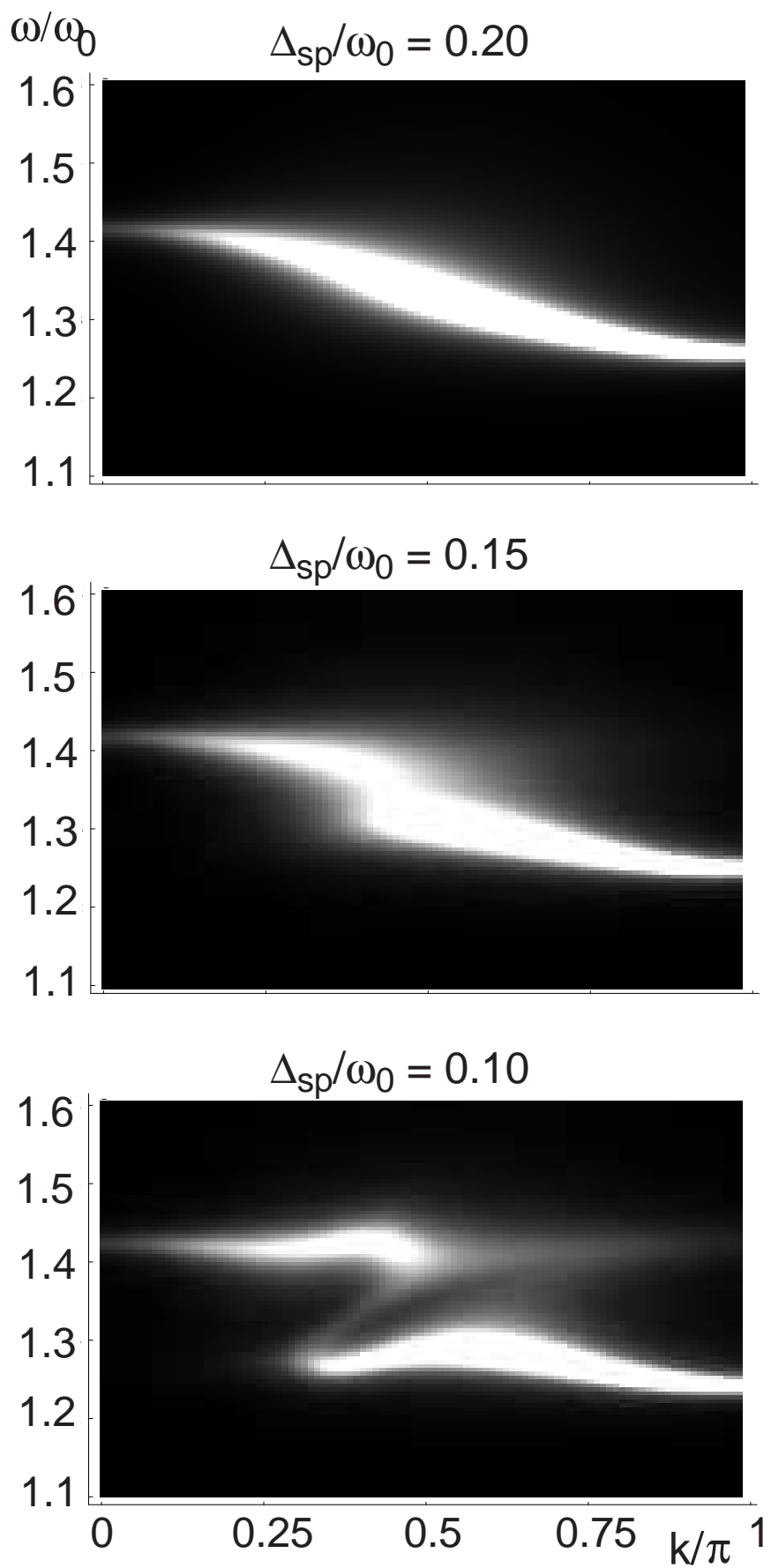

FIG. 12. Plot of the phonon spectral density $\operatorname{Im} D(k, \omega)$ defined in (25). The figure shows the influence of the spin-Peierls fluctuations described by (22) on the optical phonon mode A-B in Fig 11; the two degrees of freedom are coupled by the non-linear terms in (24). We used the parameters $\omega_{0} \equiv \sqrt{K /\left(1 / M_{\mathrm{Cu}}+1 / M_{\mathrm{O}}\right)}, \Gamma_{\mathrm{sp}}=0.5 \omega_{0}, T=0.1 \omega_{0}$, $c=10.0 \omega_{0}$, and $\gamma_{1}=\gamma_{2}=0.5 K \omega_{0}$. The figures show the evolution in the spectrum as a function of $\Delta_{\mathrm{sp}} / \omega_{0}$.
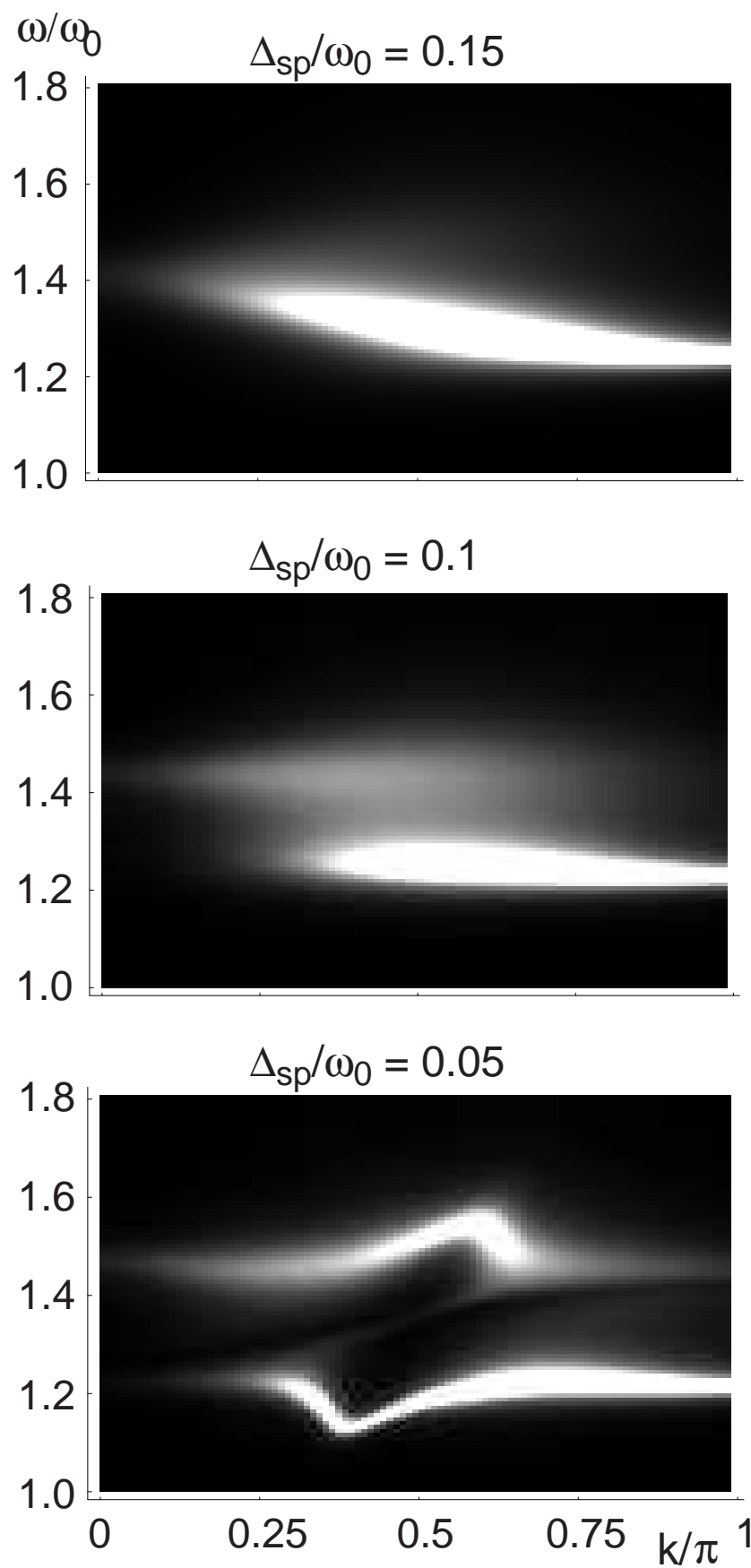

FIG. 13. As in Fig 12 but for $\Gamma_{\mathrm{sp}}=0.2 \omega_{0}, T=0.1 \omega_{0}$, $c=5.0 \omega_{0}$, and $\gamma_{1}=\gamma_{2}=0.5 K \omega_{0}$. 
are shown in Figs 12 and 13 for a representative set of parameters. As $\Delta_{\mathrm{sp}}$ decreases, there is initially a broadening and a sharpening of the phonon mode near $k=\pi / 2$. For even smaller $\Delta_{\text {sp }}$ we see the incipient Brillouin zone boundary of the doubled unit cell developing near $k=\pi / 2$ : this is reflected in the apparent discontinuity of the dispersion and the appearance of "shadow" phonon bands reflected across the incipient Brillouin zone boundary. The first of these features is in excellent accord with all the available neutron scattering experiments21 49,51 , and there has even been a claim of the observation of shadow bands21, although this has not been confirmed.

Another interesting feature of Figs 12 and 13 is that the intensity of the phonon is larger near the Brillouin zone boundary. This is a 'form factor' effect, and arises primarily from the interplay of the different scattering lengths in (25), and the distribution of the phonon normal mode between the $\mathrm{O}$ and $\mathrm{Cu}$ sites. Curiously, precisely such a dominance $\rho$ intensity near $k=\pi$ is observed in the experiments 2151 .

\section{CONCLUSIONS}

This paper has introduced a bond operator formalism to represent the degrees of freedom of doped antiferromagnets. While the approach bears some similarities to the popular "slave" boson and fermion techniques, the implementation leads to theories with a rather different structure: there are no long-range gauge forces in the fluctuations about any reasonable saddle-point in the latter formalism, and the quantum numbers of the true elementary excitations are simply connected to those of the microscopic operators. This is a powerful advantage of the bond operator method, and allows much information to be gleaned from simple mean-field Hatree-Fock-BCS computations. The main disadvantage of the method is that it requires a pairing of the sites into bonds at the outset. Such a pairing is naturally present in systems with spontaneous or explicit bond-centered charge order (as in a spin-Peierls states), and it is for these systems that the approach is best suited.

The defining equations of the bond operator formalism were presented in Section II, and are contained in Eqns (1-6). Next, we applied this formalism to the twodimensional doped antiferromagnet sketched in Fig 1 b. The main phase diagram of the model is sketched in Fig 2, and the important properties of the phases are summarized in its caption. The remaining figures in Fig II and their captions summarizing the excitation spectra of the phases - the fermionic, $S=1 / 2$, spectra are in Figs 4 , 5, 6, and 7, while the $S=1$ excitations are in Figs 8 and 9 ; the latter consist of a bosonic, $S=1$ exciton, and the two-particle continua of the fermionic $S=1 / 2$ excitations.

The connection of these result to recent neutron scat- tering measurements of phonon spectra 21, 49 , 51 was considered in Section V. This section did not use the bond operator formalism. Instead, it considered the consequences of incipient bond-centered charge order in an isotropic state, in a simple perturbative calculation using a spin-phonon model. We introduced an order parameter, $\Psi_{\mathrm{sp}}$, characterizing the ordering pattern associated with Fio $1 \mathrm{~b}$, and wrote down a phenomenological free energy 44 describing its fluctuations in spacetime in the vicinity of the quantum critical point labeled by the point $\mathrm{C}$ in Fig 2. On the symmetric side of $\mathrm{C}$ (the region with $\left\langle\Psi_{\mathrm{sp}}\right\rangle=0$, , these fluctuations were controlled by an energy scale, $\Delta_{\mathrm{sp}}$, which vanished as $\mathrm{C}$ was approached, and we considered the evolution of the optical phonon spectra as a function of decreasing $\Delta_{\mathrm{sp}}$ : these results are contained in Figs 12 and 13.

In the unifying language introduced by Nayak 48 , the bond-centered charge order parameter $\Psi_{\mathrm{sp}}$ may be considered as the amplitude of a $p_{x}$ density wave at wavevector $(\pi, 0)$. The ordinary $s$ density wave at wavevector $(\pi, 0)$ is associated with $\mathrm{Cu}$ site-centered charge order, and these may also be strong in the lightly doped antiferromagnet, especially in the region with long-range magnetic order. Their influence on the optical phonon spectra was considered in Ref 52 the primary effect was a broadening of the $\mathrm{O}$ optical phonon near $(\pi, 0)$. This should be contrasted with the influence of the $\Psi_{\text {sp }}$ fluctuations described above - the strongest effect was near $(\pi / 2,0)$ where the phonon dispersion sharpened considerably, along with a significant amount of broadening. The latter effects areclearly seen in recent neutron scattering experiment 21,49, although some softening at $(\pi, 0)$ is also apparent 51. We hope that higher precision and more detailed neutron scattering experiments will be undertaken, and along with more microscopic theoretical computations, these should help sort out the relative roles of site- and bond- centered charge order as a function of increasing doping.

In addition to fluctuating charge-order modes detected in phonon scattering, it would also be useful to study systems in which the charge order is required to be static; in such situations, atomic resolution STM studies should yield much useful information on the microstructure of the charge order. Our physical picture implies that static charge order should be present in situations in which both magnetic and superconducting order have been suppressed (systems with one of these orders may only have fluctuating charge order). A convenient way to achieve this is by application of a strong magnetic field on underdoped samples 58. A phenomenological theory of the phase diagram in a magnetic field has been provided recently in Ref 59: the "normal" state in this phase diagram is a very attractive candidate to bond-centered charge order. It would be especially interesting to conduct STM measurements on the strongly underdoped YBCO crystals that have become available recently 60 , after superconductivity has been suppressed by a static magnetic field. An alternative is to look for charge or- 
der in STM studies in which the superconductivity has only been locally suppressed, as is the case in the cores of vortices in the superconducting order 61.62 . However, the short-range nature of the suppression means that charge order is not required to appear, and may remain dynamic - this makes this approach less attractive. Recent indication 63 of mesoscale self-segregation of charge carriers in bulk samples also naturally raise the possibility of bond charge order in the lower density regions which (presumably) have suppressed superconductivity.

\section{A. Review of related work}

Before closing this paper, we present brief critical reviews of some related work in the literature, and relate it our results.

Sushkov and collaborators have studied aspects of Mott insulators both at zero and finite doping. At zero doping, they have examined the square lattice $S=1 / 2$ Heisenbserg antiferromagnet with first $\left(J_{1}\right)$ and second $\left(J_{2}\right)$ neighbor exchange 64: with increasing $J_{2} / J_{1}$ they find a transition from the familiar Néel state to a paramagnetic state with bond-centered charge order as in Fig $1 \mathrm{~b}$, albeit with a small window of parameters at which the two orders co-exist; such a co-existence region is allowed in the framework of the earlier field-theoretic analysis 6 . At somewhat larger $J_{2} / J_{1}$ they find further charge ordering which breaks an additional $Z_{2}$ lattice symmetry: this pattern of charge ordering has also been seen in recent DMRG studies 65 . Sushkov 41 has also studied the evolution of the ground state as a function of doping: by a series expansion method he finds that after destruction of the Neel order, the ground state has the columnar spin-Peierls order of Fig I 1 b. Only at a significantly larger coupling is the full square lattice symmetry restored. All of these results are fully consistent with the approach outlined in our paper.

Mazumdar, Clay, and Campbell 66 68 have studied two-dimensional correlated electron models associated with the organic charge-transfer solids tetramethyl-tetrathiafulvalene (TMTTF), tetramethyl-tetraselenafulvalene (TMTSF), bisethylenedithio-tetrathiafulvalene (BEDT-TTF) and bisethylenedithio-tetraselenafulvalene (BETS). They have argued that these materials are characterized by quarter-filled band, and produced evidence for "bond order wave" in their models at quarter filling. These states are closely related to the period 4 bond-centered charge density wave state studied in Ref7, and so also to the insulating and superconducting states with the symmetry of Fig $1 \mathrm{~b}$ studied here.

Kivelson, Fradkin and Emery 69 have studied symmetry breaking in two-dimensional electronic systems using a rather general classification in terms of electronic liquid crystal phases. Their formulation (and their earlier theory 70 of the "spin gap proximity effect") does not distinguish between the nature of bond- and site- centered charge order that we are paying particular attention to here. A significant portion of the superconducting state in their phase diagram overlaps with a region of nematic order. We believe that the orthorhombic anisotropy of this nematic order is ultimately driven by singlet bond correlations associated with the spin-Peierls order in Fig 1 l b; consequently this nematic region should show bond-centered smectic correlations with a period of 2 lattice spacings, as has been claimed in the experiments of Ref 21. We make this distinction more precise by discussing how the spin-Peierls and nematic ordersare coupled. We can characterize the spin-Peierls order 11 in Fig 1 b by a complex $Z_{4}$ order parameter $\Psi_{\mathrm{sp}}$ which takes the values $1, i,-1,-i$ on the four states obtained by rotating Fig $1 \mathrm{~b}$ about any lattice site successively by $90^{\circ}$. Similarly, we can introduce a real Ising nematic order parameter $\Phi_{\mathrm{n}}$ which is $+1(-1)$ for a nematic polarized along the $x(y)$ direction. Then the symmetry properties of the two order parameters show that 1

$$
\Phi_{\mathrm{n}} \sim \Psi_{\mathrm{sp}}^{2}
$$

A detailed Landau theory and fluctuation analysis of the interplay between these two order parameters is presented in Appendix A. It is our picture, based on the physical arguments above, that $\Psi_{\mathrm{sp}}$ is the primary order parameter, and that $\Phi_{\mathrm{n}}$ is tied to it via (26).

An important ingredient in the work of Carlson, Orgad, Kivelson, and Emery 72 id is the crossover from the physics of a one-dimensional electron gas moving in the vertical direction in Fig $1 \mathrm{~b}$ at short scales, to a coupled two-dimensional system at long scales. This should be contrasted from our approach, in which we do not find any quasi-one-dimensional regime. Although the lattice symmetry of our states is similar to those considered by these authors, the physics is always intrinsically twodimensional, albeit with a spatial anisotropy. Indeed, the arguments for confinement and bond-centered charge order rely crucially on the two-dimensionality of the system.

Zaanen and collaborators 3 have recently given a bold physical picture of the microstructure of stripes and their relationship to high temperature superconductivity. They take a solitonic, low-dimensional perspective, in which special attention is paid to excitations at various boundaries and dislocations in the stripe order. In contrast, our perspective here is a higher (two-) dimensional point of view, appropriate to systems not too far below their upper-critical dimension. So e.g. we view the spinPeierls order parameter $\Psi_{\mathrm{sp}}$ as a "soft-spin" field with large and continuous variations in its local amplitude, rather than a field with 4 discrete possible values in different regions of space separated by identifiable domain walls. The advantage of our perspective is that it allows a complete (in principle) and systematic treatment of the coupling of fermionic degrees of freedom to the various order 埇ameters in a Landau-theory like scattering framework 4 . 
White and Scalapino 75 have presented results for charge order in DMRG studies of doped antiferromagnets. They find that site and bond centered stripes are almost degenerate in their energy. They do observe a period 4 bond-centered charge stripe stated, but have not so far seen a state with the period 2 symmetry of Fig $11 \mathrm{~b}$.

Castro Neto 6 has recently presented an interesting analysis of a striped superconductor, coupling transverse stripe fluctuations between regions of high and low hole density. Notably, he also finds pronounced tendency towards bond-centered charge order with the symmetry of Fig 1 b.

Lannert, Fisher, and Senthil 77 have considered Berry phase effects in a $Z_{2}$ gauge theory of correlated electron systems. Close to half-filling, they find that these Berry phases induce bond-centered charge order in both insulating and superconducting phases, in a manner closely analogous to that found in Ref 4 in paramagnetic Mott insulators. They also discuss the critical properties of a transition between superconducting and insulating states at half filling, with both phases containing a background charge order with the symmetry of Fig 1 $1 \mathrm{~b}$. They pay particular attention to the influence of the gapless nodal fermions on the vortices in the superconducting order. However, in the presence of the background charge order at wavevector $(\pi, 0)$, their nodal fermions (which are at $( \pm \pi / 2, \pm \pi / 2))$ should immediately acquire a gap, and their theory then reduces to a dualized version of familiar bosonic theory of the superconductor-insulator transition.

\section{ACKNOWLEDGMENTS}

We thank G. Aeppli, M. Braden, D. Campbell, E. Carlson, T. Egami, S. Girvin, E. Fradkin, S. Kivelson, A. Lanzara, A. Polkovnikov, T. Senthil, Z.-X. Shen, M. Vojta and J. Zaanen for useful discussions. We are especially grateful to $\mathrm{S}$. Kivelson for a critical reading of the manuscript. This research was supported by US NSF Grant DMR 0098226.

\section{APPENDIX A: INTERPLAY OF SPIN PEIERLS AND NEMATIC ORDERS}

In Section If we discussed the relationship between states with spin-Peierls order, with the symmetry of Fig 1 $1 \mathrm{~b}$, and the nematic electronic states discussed by Kivelson, Fradkin and Emery 69 . We noted that the spin-Peierls stafe was characterized by a complex order parameter $71 \Psi_{\mathrm{sp}}$ (which took the values $1, i,-1$, $-i$ on the four states obtained by rotating Fig 1 b about any lattice site), the nematic was characterized by a real Ising order parameter $\Phi_{\mathrm{n}}$, and the symmetry properties of these two order parameters implied the relationship (26) between them. More generally, we can write down the following simple effective action for them, keeping all low order terms consistent with the underlying symmetries:

$$
\begin{aligned}
S_{\mathrm{sp}-\mathrm{n}} & =\int_{0}^{1 / T} d \tau \int d^{2} x\left[\left|\partial_{\tau} \Psi_{\mathrm{sp}}\right|^{2}+c_{1}^{2}\left|\nabla_{x} \Psi_{\mathrm{sp}}\right|^{2}+r_{1}\left|\Psi_{\mathrm{sp}}\right|^{2}\right. \\
& +\frac{1}{2}\left(\partial_{\tau} \Phi_{\mathrm{n}}\right)^{2}+\frac{1}{2} c_{2}^{2}\left(\nabla_{x} \Phi_{\mathrm{n}}\right)^{2}+\frac{r_{2}}{2} \Phi_{\mathrm{n}}^{2} \\
& -g \Phi_{\mathrm{n}}\left(\Psi_{\mathrm{sp}}^{2}+\Psi_{\mathrm{sp}}^{* 2}\right)+\frac{u_{1}}{2}\left|\Psi_{\mathrm{sp}}\right|^{4}-\frac{v}{4}\left(\Psi_{\mathrm{sp}}^{4}+\Psi_{\mathrm{sp}}^{* 4}\right) \\
& \left.-\frac{w}{2} \Phi_{n}^{2}\left|\Psi_{\mathrm{sp}}\right|^{2}+\frac{u_{2}}{4} \Phi_{\mathrm{n}}^{4}\right]
\end{aligned}
$$

where $\tau$ is imaginary time, and $u_{1}>v>0, u_{2}>0$, and $u_{2}\left(u_{1}-v\right)>w^{2}$ required for stability. We expect that the appearance of nematic order will enhance the probability of spin Peierls order, and so $w>0$. We have, for now, neglected the couplings of these order to the fermionic excitations, and will consider the consequences of these later in this section.

First, let us analyze $S_{\text {sp-n }}$ in mean-field theory. The results of such an analysis are shown in Fig 14 . There are three phases: (i) the symmetric phase, where no lattice symmetry is broken, (ii) the spin Peierls phase, where both the spin Peierls and nematic order parameters are non-zero, and (iii), the nematic phase, where only the nematic order is non-zero. There can be a second-order phase transition between any two of these three phases, as indicated in Fig 14. The position of these second-order boundaries can be determined by a conventional Landau theory analysis in powers of the associated order parameter. In this manner, we find that the second-order line between the symmetric phase and the spin-Peierls phase is at $r_{1}=0$, while that between the symmetric phase and the nematic phase is at $r_{2}=0$. Finally, the second-order transition from the nematic to the spin-Peierls phase is at

$$
r_{1}=2 g \sqrt{-r_{2} / u_{2}}-w r_{2} /\left(2 u_{2}\right)
$$

Notice that these three second-order lines all appear to meet at the origin $r_{1}=0, r_{2}=0$. However, as indicated in Fig 14, this is pre-empted by a line of first order transitions close to the origin. The reason for this may be seen by the following simple argument. Imagine integrating out the $\Phi_{\mathrm{n}}$ fluctuations to derive an effective action for the $\Psi_{\mathrm{sp}}$ : this always induces an effective quartic term $\sim\left|\Psi_{\mathrm{sp}}\right|^{4}$ with a coefficient $\sim-g^{2} /\left|r_{2}\right|$. So for small enough $\left|r_{2}\right|$, the net coefficient of $\left|\Psi_{\text {sp }}\right|^{4}$ always becomes negative, and this drives the transitions involving onset of non-zero $\left\langle\Psi_{\mathrm{sp}}\right\rangle$ first order. A consequence of this line of first-order transitions is that there are two tricritical points $T_{1}, T_{2}$, and their positions are indicated in the caption to Fig 14 .

Let us now consider the nature of the fluctuations in the vicinity of the second-order phase transitions. We examine first the transition from the symmetric to the 

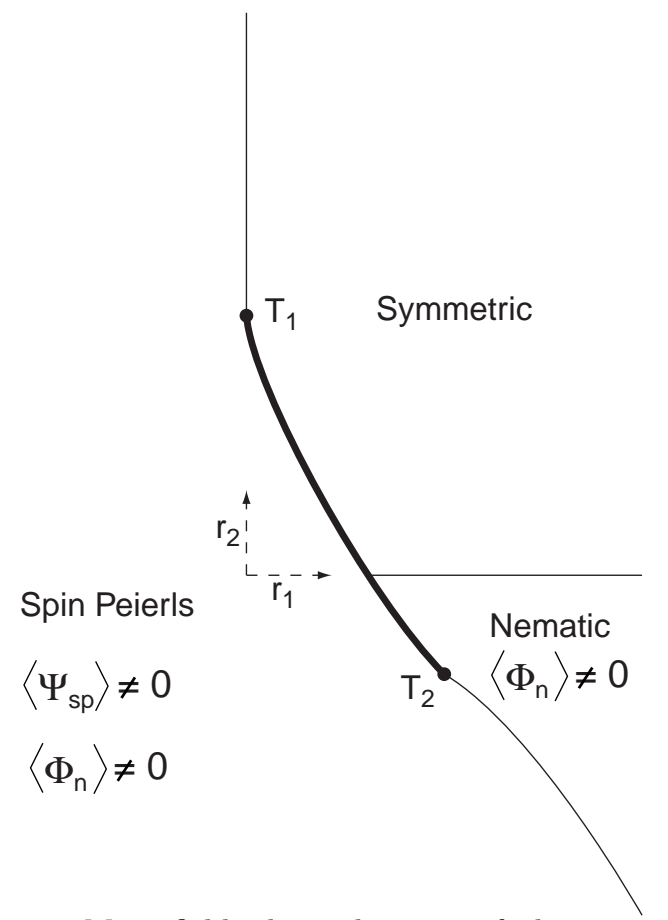

FIG. 14. Mean-field phase diagram of the action $S_{\mathrm{sp}-\mathrm{n}}$ in $\mathrm{A} 1$. The symmetric phase has no broken lattice symmetries. The thin (thick) lines represent second (first) order transitions. The positions of the second order transition lines is indicated in A2 and above. The tricritical point $T_{1}$ is at $r_{1}=0, r_{2}=4 g^{2} /\left(u_{1}-v\right)$, while the tricritical point $T_{2}$ is at the $r_{2}<0$ which is a solution of $2 u_{2} g^{2}+4 g w \sqrt{-r_{2} / u_{2}}+r_{2}\left(u_{2}\left(u_{1}-v\right)-w^{2} / 2\right)=0$, and $r_{1}$ given by (A2) for this value of $r_{2}$. At $T=0$, the coupling to the nodal fermions in a $d$-wave superconductor drives the transition from the symmetric to the nematic phase first order 74 nematic phase, which has an Ising order parameter. For $T>0$, these transitions will remain in the universality class of the two-dimensional Ising model. However, at $T=0$, we have to also consider the influence of the fermionic excitations. A general analysis of such effects has been presented in Ref 74, and we recall the results relevant to our discussion here. The $T=0$ ground state is either an insulator or a superconductor, and only the gapless nodal fermionic excitations can influence the nature of the critical properties of a zero temperature quantum phase transition. In an anisotropic, $d$-wave superconductor, the four nodal points are described by a Dirac-like Hamiltonian for 2, four-component, fermionic Nambu spinors $\Psi_{1}, \Psi_{2}$ (we will follow the notation of Ref 74. The nematic order parameter has zero net momentum, and so has a simple linear coupling to these fermionic excitation 74 :

$$
\Phi_{\mathrm{n}}\left(\Psi_{1}^{\dagger} \tau^{x} \Psi_{1}+\Psi_{2} \tau^{x} \Psi_{2}\right)
$$

where $\tau^{x, y, z}$ are Pauli matrices in the Nambu space (again, in the notation of Ref 74). A renormalization group analysis of the consequences of (A3) has been carried out, and it is found that the couplings to the fermions drives the $T=0$ transition between the symmetric and nematic phases first order.

Now let us consider the remaining two second-order transitions in Fig 14, for which $\Psi_{\text {sp }}$ is the order parameter. In contrast to $\Phi_{\mathrm{n}}$, the spin-Peierls order has momentum $(\pi, 0)$ or $(0, \pi)$, and so has no simple linear coupling to the nodal points, barring the exceptional case in which the nodal points are exactly at $(\pi / 2, \pi / 2)$. In general, the couplings between $\Psi_{\mathrm{sp}}$ and the fermions are of the form

$$
\Psi_{\mathrm{sp}}^{2}\left(\Psi_{1}^{\dagger} \tau^{x} \Psi_{1}+\Psi_{2} \tau^{x} \Psi_{2}\right)
$$

as can be expected from (26) and (A3), and also of the form

$$
\left|\Psi_{\mathrm{sp}}\right|^{2}\left(\Psi_{1}^{\dagger} \tau^{z} \Psi_{1}+\Psi_{2} \tau^{z} \Psi_{2}\right),
$$

Unlike (A3), the couplings in (A4, A5) are irrelevant, and so the fermions do not modify the leading critical properties of any transition in which $\Psi_{\mathrm{sp}}$ is the order parameter.

For the transition between the nematic and spinPeierls phases, the order parameter is either the real or imaginary part of $\Psi_{\mathrm{sp}}$ : the presence of nematic order in both phases therefore makes the residual order parameter Ising-like. So this transition is in the universality class of three-dimensional (two-dimensional) Ising model at $T=0(T>0)$.

Finally, we consider the fluctuations near the secondorder transition between the symmetric and the spinPeierls phase. As we have just noted, the fermionic excitations can be neglected even at $T=0$. Further, as $\Psi_{\mathrm{sp}}$ is the primary order parameter, we can integrate out the $\Phi_{\mathrm{n}}$ fluctuations at the cost of modifying the couplings in 
the effective action for the $\Psi_{\mathrm{sp}}$ alone. The resulting action has the same form as $S$, after dropping all the terms involving $\Phi_{\mathrm{n}}$; so near the critical point, we need only follow the flow of the nonlinear couplings $u_{1}$ and $v$. For the $T=0$ transition, the standard Wilson-Fisher analysis of a phase transition in three spacetime dimension leads to the renormalization group flow equations

$$
\begin{aligned}
\frac{d u_{1}}{d \ell} & =u_{1}-5 u_{1}^{2}-9 v^{2} \\
\frac{d v}{d \ell} & =v-6 u_{1} v
\end{aligned}
$$

where we have absorbed a phase-space factor by a rescaling of the couplings in a standard manner. The only stable fixed point of these equations is $u_{1}=1 / 5, v=0$. At the point $v=0$, the effective action for $\Psi_{\mathrm{sp}}$ has the additional symmetry under the global $U(1)$ change in the phase of $\Psi_{\mathrm{sp}}$. So the $T=0$ critical point is in the universality class of three dimensional XY model. Related considerations can be applied to the $T>0$ transition between the symmetric and spin-Peierls phases: there, the anisotropy associated with $v$ leads to phase transitions with continuously varying exponents, associated with physics of the Ashkin-Teller model 78 .

We conclude this section by summarizing the main physical implications of Fig 14. Starting from the symmetric phase, one may break a square lattice symmetry either by lowering temperature, or by reducing doping. One route to this is a direct second-order transition from the symmetric to the spin-Peierls phase; the latter phase also has nematic order. The implications of the physical arguments we have presented in Section I is that this is the preferred route. Alternatively, we can first undergo a transition to the nematic phase, and possibly have a further symmetry breaking into the spin-Peierls phase.

\section{APPENDIX B: COMPUTATIONS FOR THE TWO-LEG LADDER}

We begin a bond operator theory of doped two-leg ladder by writing down the Hamiltonian for the pure t-J model. The effect of Coulomb repulsion will be considered in Appendix D.

$$
\begin{aligned}
H & =H_{t}+H_{J} \\
& =-t \sum_{i}\left\{c_{1 i a}^{\dagger} c_{2 i a}+c_{2 i a}^{\dagger} c_{1 i a}\right\} \\
& -t \sum_{\langle i, j\rangle}\left\{c_{1 j a}^{\dagger} c_{1 i a}+c_{2 j a}^{\dagger} c_{2 i a}+(\text { h.c. })\right\} \\
& +J \sum_{i} \mathbf{S}_{1 i} \cdot \mathbf{S}_{2 i} \\
& +\lambda J \sum_{\langle i, j\rangle}\left\{\mathbf{S}_{1 i} \cdot \mathbf{S}_{1 j}+\mathbf{S}_{2 i} \cdot \mathbf{S}_{2 j}\right\}
\end{aligned}
$$

where $i$ indicates the position of the $i$-th dimer along the ladder direction and $\langle i, j\rangle$ stands for the summation over the nearest neighbor, i.e. $j=i+1$.

First, let us take a look at the hopping Hamiltonian $H_{t}$. Hopping between the sites inside a given dimer is given by:

$$
c_{1 i a}^{\dagger} c_{2 i a}+c_{2 i a}^{\dagger} c_{1 i a}=h_{1 i a}^{\dagger} h_{2 i a}+h_{2 i a}^{\dagger} h_{1 i a}
$$

Hopping between the sites of adjacent dimers is obtained as follows.

$$
\begin{aligned}
c_{1 i a}^{\dagger} c_{1 j a} & =\left\{h_{1 i a}^{\dagger} d_{i}+\frac{1}{\sqrt{2}} \varepsilon_{a b} s_{i}^{\dagger} h_{2 i b}-\frac{1}{\sqrt{2}} \varepsilon_{a c} \sigma_{c b}^{\alpha} t_{i \alpha}^{\dagger} h_{2 i b}\right\} \\
& \times\left\{d_{j}^{\dagger} h_{1 j a}+\frac{1}{\sqrt{2}} \varepsilon_{a c} h_{2 j c}^{\dagger} s_{j}-\frac{1}{\sqrt{2}} \varepsilon_{a e} \bar{\sigma}_{e d}^{\beta} h_{2 j d}^{\dagger} t_{j \beta}\right\} \\
& \Rightarrow h_{1 i a}^{\dagger} h_{1 j a} d_{i} d_{j}^{\dagger}+\frac{1}{2} \varepsilon_{a b} \varepsilon_{a c} s_{i}^{\dagger} s_{j} h_{2 i b} h_{2 j c}^{\dagger} \\
& +\frac{1}{\sqrt{2}} d_{i} s_{j} \varepsilon_{a c} h_{1 i a}^{\dagger} h_{2 j c}^{\dagger}+\frac{1}{\sqrt{2}} d_{j}^{\dagger} s_{i}^{\dagger} \varepsilon_{a b} h_{2 i b} h_{1 j a} \\
& +\frac{1}{2} \varepsilon_{a c} \varepsilon_{a e} \sigma_{c b}^{\alpha} \bar{\sigma}_{e d}^{\beta} t_{i \alpha}^{\dagger} t_{j \beta} h_{2 i b} h_{2 j d}^{\dagger}
\end{aligned}
$$

where any term containing a sigle $t$ boson operator is ignored assuming that there is no magnetic order. Similarly,

$$
\begin{aligned}
c_{2 i a}^{\dagger} c_{2 j a} & =\left\{h_{2 i a}^{\dagger} d_{i}+\frac{1}{\sqrt{2}} \varepsilon_{a b} s_{i}^{\dagger} h_{1 i b}+\frac{1}{\sqrt{2}} \varepsilon_{a c} \sigma_{c b}^{\alpha} t_{i \alpha}^{\dagger} h_{1 i b}\right\} \\
& \times\left\{d_{j}^{\dagger} h_{2 j a}+\frac{1}{\sqrt{2}} \varepsilon_{a c} h_{1 j c}^{\dagger} s_{j}+\frac{1}{\sqrt{2}} \varepsilon_{a e} \bar{\sigma}_{e d}^{\beta} h_{1 j d}^{\dagger} t_{j \beta}\right\} \\
& \Rightarrow h_{2 i a}^{\dagger} h_{2 j a} d_{i} d_{j}^{\dagger}+\frac{1}{2} \varepsilon_{a b} \varepsilon_{a c} s_{i}^{\dagger} s_{j} h_{1 i b} h_{1 j c}^{\dagger} \\
& +\frac{1}{\sqrt{2}} d_{i} s_{j} \varepsilon_{a c} h_{2 i a}^{\dagger} h_{1 j c}^{\dagger}+\frac{1}{\sqrt{2}} d_{j}^{\dagger} s_{i}^{\dagger} \varepsilon_{a b} h_{1 i b} h_{2 j a} \\
& +\frac{1}{2} \varepsilon_{a c} \varepsilon_{a e} \sigma_{c b}^{\alpha} \bar{\sigma}_{e d}^{\beta} t_{i \alpha}^{\dagger} t_{j \beta} h_{1 i b} h_{1 j d}^{\dagger}
\end{aligned}
$$

By combining the above two equations, we get

$$
\begin{aligned}
& c_{1 i a}^{\dagger} c_{1 j a}+c_{2 i a}^{\dagger} c_{2 j a}+(\text { h.c. }) \\
= & d_{i} d_{j}^{\dagger}\left\{h_{1 i a}^{\dagger} h_{1 j a}+h_{2 i a}^{\dagger} h_{2 j a}\right\} \\
+ & \frac{1}{2} s_{i}^{\dagger} s_{j} \varepsilon_{a b} \varepsilon_{a c}\left\{h_{1 i b} h_{1 j c}^{\dagger}+h_{2 i b} h_{2 j c}^{\dagger}\right\} \\
+ & \frac{1}{\sqrt{2}} d_{i} s_{j} \varepsilon_{a c}\left\{h_{2 i a}^{\dagger} h_{1 j c}^{\dagger}+h_{1 i a}^{\dagger} h_{2 j c}^{\dagger}\right\} \\
+ & \frac{1}{\sqrt{2}} d_{j}^{\dagger} s_{i}^{\dagger} \varepsilon_{a b}\left\{h_{1 i b} h_{2 j a}+h_{2 i b} h_{1 j a}\right\} \\
+ & \frac{1}{2} \varepsilon_{a c} \varepsilon_{a e} \sigma_{c b}^{\alpha} \bar{\sigma}_{e d}^{\beta} t_{i \alpha}^{\dagger} t_{j \beta}\left\{h_{1 i b} h_{1 j d}^{\dagger}+h_{2 i b} h_{2 j d}^{\dagger}\right\}+(h . c .)
\end{aligned}
$$

Assuming $s$ and $d$ bosons are condensed, i.e. $s_{i}=\bar{s}$ and $d_{i}=\bar{d}$, one is able to express $H_{t}$ as follows.

$$
H_{t}=-t \sum_{i}\left\{h_{1 i a}^{\dagger} h_{2 i a}+h_{2 i a}^{\dagger} h_{1 i a}\right\}
$$




$$
\begin{aligned}
- & t\left(\bar{d}^{2}-\frac{1}{2} \bar{s}^{2}\right) \sum_{\langle i, j\rangle}\left\{h_{1 i a}^{\dagger} h_{1 j a}+h_{1 j a}^{\dagger} h_{1 i a}\right. \\
& \left.+h_{2 i a}^{\dagger} h_{2 j a}+h_{2 j a}^{\dagger} h_{2 i a}\right\} \\
- & \sqrt{2} t \bar{d} \bar{s} \sum_{\langle i, j\rangle} \varepsilon_{a b}\left\{h_{1 i a}^{\dagger} h_{2 j b}^{\dagger}\right. \\
& \left.+h_{2 i a}^{\dagger} h_{1 j b}^{\dagger}+h_{2 j b} h_{1 i a}+h_{1 j b} h_{2 i a}\right\} \\
+ & H_{t^{2} h^{2}}
\end{aligned}
$$

where

$$
\begin{aligned}
H_{t^{2} h^{2}} & =-\frac{t}{2} \varepsilon_{a c} \varepsilon_{a e} \sigma_{c b}^{\alpha} \sigma_{e d}^{\beta} \sum_{\langle i, j\rangle}\left\{t_{i \alpha}^{\dagger} t_{j \beta}\left(h_{1 i b} h_{1 j d}^{\dagger}+h_{2 i b} h_{2 j d}^{\dagger}\right)\right. \\
& +(\text { h.c. })\}
\end{aligned}
$$

Now let us turn our attention to the Hamiltonian $H_{J}$. Contribution from the coupling inside the dimer has been shown previously. That is,

$$
\mathbf{S}_{1 i} \cdot \mathbf{S}_{2 i}=-\frac{3}{4} s_{i}^{\dagger} s_{i}+\frac{1}{4} t_{i \alpha}^{\dagger} t_{i \alpha}
$$

Coupling term between the spins of different dimers is given by:

$$
\begin{aligned}
S_{1 i \alpha} S_{1 j \alpha} & =\tilde{S}_{1 i \alpha} \tilde{S}_{1 j \alpha}+\frac{1}{4} \sigma_{a b}^{\alpha} \sigma_{c d}^{\alpha} h_{1 i a}^{\dagger} h_{1 i b} h_{1 j c}^{\dagger} h_{1 j d} \\
& +\frac{1}{2} h_{1 i a}^{\dagger} \sigma_{a b}^{\alpha} h_{1 i b} \tilde{S}_{1 j \alpha}+\frac{1}{2} h_{1 j a}^{\dagger} \sigma_{a b}^{\alpha} h_{1 j b} \tilde{S}_{1 i \alpha} .
\end{aligned}
$$

Remember that $\tilde{S}_{1 i \alpha}=\frac{1}{2}\left(s_{i}^{\dagger} t_{i \alpha}+t_{i \alpha}^{\dagger} s_{i}-i \epsilon_{\alpha \beta \gamma} t_{i \beta}^{\dagger} t_{i \gamma}\right)$ and $\tilde{S}_{2 i \alpha}=-\frac{1}{2}\left(s_{i}^{\dagger} t_{i \alpha}+t_{i \alpha}^{\dagger} s_{i}+i \epsilon_{\alpha \beta \gamma} t_{i \beta}^{\dagger} t_{i \gamma}\right)$. The cross term can be ignored assuming that there is no contribution from the term containing a single $t$ operator or $t_{\alpha}^{\dagger} t_{\beta}$ with $\alpha \neq \beta$. In other words, it is assumed that there is no magnetic ordering. Therefore,

$$
\begin{aligned}
& S_{1 i \alpha} S_{1 j \alpha}+S_{2 i \alpha} S_{2 j \alpha} \\
= & \tilde{S}_{1 i \alpha} \tilde{S}_{1 j \alpha}+\tilde{S}_{2 i \alpha} \tilde{S}_{2 j \alpha}+\frac{1}{\lambda J} H_{h^{4}}
\end{aligned}
$$

where

$$
\begin{aligned}
H_{h^{4}} & =\frac{\lambda J}{4} \sum_{\langle i, j\rangle} \sigma_{a b}^{\alpha} \sigma_{c d}^{\alpha}\left\{h_{1 i a}^{\dagger} h_{1 i b} h_{1 j c}^{\dagger} h_{1 j d}\right. \\
& \left.+h_{2 i a}^{\dagger} h_{2 i b} h_{2 j c}^{\dagger} h_{2 j d}\right\}
\end{aligned}
$$

Coupling terms involving $\tilde{S}$ are evaluated below.

$$
\begin{aligned}
4\left(\tilde{S}_{1 i \alpha} \tilde{S}_{1 j \alpha}+\right. & \left.\tilde{S}_{2 i \alpha} \tilde{S}_{2 j \alpha}\right) \\
= & \left(s_{i}^{\dagger} t_{i \alpha}+t_{i \alpha}^{\dagger} s_{i}-i \epsilon_{\alpha \beta \gamma} t_{i \beta}^{\dagger} t_{i \gamma}\right) \\
& \times\left(s_{j}^{\dagger} t_{j \alpha}+t_{j \alpha}^{\dagger} s_{j}-i \epsilon_{\alpha \mu \nu} t_{j \mu}^{\dagger} t_{j \nu}\right) \\
+ & \left(s_{i}^{\dagger} t_{i \alpha}+t_{i \alpha}^{\dagger} s_{i}+i \epsilon_{\alpha \beta \gamma} t_{i \beta}^{\dagger} t_{i \gamma}\right) \\
& \times\left(s_{j}^{\dagger} t_{j \alpha}+t_{j \alpha}^{\dagger} s_{j}+i \epsilon_{\alpha \mu \nu} t_{j \mu}^{\dagger} t_{j \nu}\right) \\
= & 2\left\{t_{i \alpha}^{\dagger} t_{j \alpha}^{\dagger} s_{i} s_{j}+t_{i \alpha} t_{j \alpha} s_{i}^{\dagger} s_{j}^{\dagger}\right. \\
& \left.+t_{i \alpha}^{\dagger} t_{j \alpha} s_{j}^{\dagger} s_{i}+t_{j \alpha}^{\dagger} t_{i \alpha} s_{i}^{\dagger} s_{j}\right\} \\
- & 2 \epsilon_{\alpha \beta \gamma} \epsilon_{\alpha \mu \nu} t_{i \beta}^{\dagger} t_{i \gamma} t_{j \mu}^{\dagger} t_{j \nu}
\end{aligned}
$$

Therefore $H_{J}$ is summarized as follows.

$$
\begin{aligned}
H_{J}= & J \sum_{i}\left\{-\frac{3}{4} s_{i}^{\dagger} s_{i}+\frac{1}{4} t_{i \alpha}^{\dagger} t_{i \alpha}\right\} \\
+ & \frac{\lambda J}{2} \sum_{\langle i, j\rangle}\left\{t_{i \alpha}^{\dagger} t_{j \alpha}^{\dagger} s_{i} s_{j}+t_{i \alpha} t_{j \alpha} s_{i}^{\dagger} s_{j}^{\dagger}\right. \\
& \left.+t_{i \alpha}^{\dagger} t_{j \alpha} s_{j}^{\dagger} s_{i}+t_{j \alpha}^{\dagger} t_{i \alpha} s_{i}^{\dagger} s_{j}\right\} \\
& -\frac{\lambda J}{2} \sum_{\langle i, j\rangle} \epsilon_{\alpha \beta \gamma} \epsilon_{\alpha \mu \nu} t_{i \beta}^{\dagger} t_{i \gamma} t_{j \mu}^{\dagger} t_{j \nu}+H_{h^{4}} \\
\Rightarrow & -\frac{3}{4} J N \bar{s}^{2}+\frac{J}{4} \sum_{i} t_{i \alpha}^{\dagger} t_{i \alpha} \\
+ & \frac{\lambda J}{2} \bar{s}^{2} \sum_{\langle i, j\rangle}\left\{t_{i \alpha}^{\dagger} t_{j \alpha}^{\dagger}+t_{i \alpha} t_{j \alpha}+t_{i \alpha}^{\dagger} t_{j \alpha}+t_{j \alpha}^{\dagger} t_{i \alpha}\right\} \\
+ & H_{t^{4}}+H_{h^{4}}
\end{aligned}
$$

where $N$ is the number of dimers and $s$ boson is condensed so that $s_{i}=\bar{s}$. The total Hamiltonian (without constraints) is given by the sum of $H_{t}$ and $H_{J}$.

$$
\begin{aligned}
H= & H_{t}+H_{J} \\
= & -t \sum_{i}\left\{h_{1 i a}^{\dagger} h_{2 i a}+h_{2 i a}^{\dagger} h_{1 i a}\right\} \\
- & t\left(\bar{d}^{2}-\frac{1}{2} \bar{s}^{2}\right) \sum_{\langle i, j\rangle}\left\{h_{1 i a}^{\dagger} h_{1 j a}+h_{1 j a}^{\dagger} h_{1 i a}\right. \\
& \left.+h_{2 i a}^{\dagger} h_{2 j a}+h_{2 j a}^{\dagger} h_{2 i a}\right\} \\
- & \sqrt{2} t \bar{d} \bar{s} \sum_{\langle i, j\rangle} \varepsilon_{a b}\left\{h_{1 i a}^{\dagger} h_{2 j b}^{\dagger}+h_{2 i a}^{\dagger} h_{1 j b}^{\dagger}\right. \\
& \left.+h_{2 j b} h_{1 i a}+h_{1 j b} h_{2 i a}\right\} \\
- & \frac{3}{4} J N \bar{s}^{2}+\frac{J}{4} \sum_{i} t_{i \alpha}^{\dagger} t_{i \alpha} \\
+ & \frac{\lambda J}{2} \bar{s}^{2} \sum_{\langle i, j\rangle}\left\{t_{i \alpha}^{\dagger} t_{j \alpha}^{\dagger}+t_{i \alpha} t_{j \alpha}+t_{i \alpha}^{\dagger} t_{j \alpha}+t_{j \alpha}^{\dagger} t_{i \alpha}\right\} \\
+ & H_{t^{2} h^{2}}+H_{t^{4}}+H_{h^{4}}
\end{aligned}
$$

As one can see in the following sections, a convenient simplification is obtained by expressing the Hamiltonian in terms of the bonding and anti-bonding fermionic operators which are defined as follows:

$$
\begin{aligned}
h_{i+a} & \equiv \frac{1}{\sqrt{2}}\left(h_{1 i a}+h_{2 i a}\right) \\
h_{i-a} & \equiv \frac{1}{\sqrt{2}}\left(h_{1 i a}-h_{2 i a}\right)
\end{aligned}
$$

\section{Mean-Field Hamiltonian without Quartic Terms}

In this section we will ignore the terms from $H_{t^{4}}, H_{h^{4}}$ and $H_{t^{2} h^{2}}$. Then, the Hamiltonian is given in momentum space representation as follows. 


$$
\begin{aligned}
& H=-N \frac{3}{4} J \bar{s}^{2}+\sum_{k_{y}}\left(\frac{J}{4}+\lambda J \bar{s}^{2} \cos k_{y}\right) t_{\alpha}^{\dagger}\left(k_{y}\right) t_{\alpha}\left(k_{y}\right) \\
& +\sum_{k_{y}} \frac{\lambda J \bar{s}^{2}}{2} \cos k_{y}\left\{t_{\alpha}^{\dagger}\left(k_{y}\right) t_{\alpha}^{\dagger}\left(-k_{y}\right)+t_{\alpha}\left(k_{y}\right) t_{\alpha}\left(-k_{y}\right)\right\} \\
& +\sum_{k_{y}}\left\{t\left(\bar{s}^{2}-2 \bar{d}^{2}\right) \cos k_{y}-t\right\} h_{+a}^{\dagger}\left(k_{y}\right) h_{+a}\left(k_{y}\right) \\
& +\sum_{k_{y}}\left\{t\left(\bar{s}^{2}-2 \bar{d}^{2}\right) \cos k_{y}+t\right\} h_{-a}^{\dagger}\left(k_{y}\right) h_{-a}\left(k_{y}\right) \\
& -2 \sqrt{2} t \bar{d} \bar{s} \sum_{k_{y}} \cos k_{y}\left\{h_{+\uparrow}^{\dagger}\left(k_{y}\right) h_{+\downarrow}^{\dagger}\left(-k_{y}\right)\right. \\
& \left.-h_{-\uparrow}^{\dagger}\left(k_{y}\right) h_{-\downarrow}^{\dagger}\left(-k_{y}\right)+(h . c .)\right\}
\end{aligned}
$$

As mentioned previously, it is necessary to impose certain constraints. In this study, constraints are introduced in Lagrange multiplier method. In other words, "constraint Hamiltonian" $\left(H_{c}\right)$ is added to the original Hamiltonian. And then the unknown parameters are determined by the saddle point condition of the ground state energy.

$$
\begin{aligned}
& H_{c}=-\mu \sum_{i}\left(s_{i}^{\dagger} s_{i}+t_{i \alpha}^{\dagger} t_{i \alpha}+h_{1 i a}^{\dagger} h_{1 i a}+h_{2 i a}^{\dagger} h_{2 i a}\right. \\
& \left.\quad+d_{i}^{\dagger} d_{i}-1\right) \\
& -\xi \sum_{i}\left(h_{1 i a}^{\dagger} h_{1 i a}+h_{2 i a}^{\dagger} h_{2 i a}+2 d_{i}^{\dagger} d_{i}-2 x\right) \\
& \Rightarrow-\mu \sum_{k_{y}}\left(t_{\alpha}^{\dagger}\left(k_{y}\right) t_{\alpha}\left(k_{y}\right)+\bar{s}^{2}-\bar{d}^{2}+2 x-1\right) \\
& -\xi \sum_{k_{y}}\left(h_{+a}^{\dagger}\left(k_{y}\right) h_{+a}\left(k_{y}\right)+h_{-a}^{\dagger}\left(k_{y}\right) h_{-a}\left(k_{y}\right)\right. \\
& \left.\quad+2 \bar{d}^{2}-2 x\right)
\end{aligned}
$$

where $x$ is the hole concentration.

Therefore the total Hamiltonian with constraints is given by:

$$
\begin{aligned}
H=N \epsilon_{0} \\
+\sum_{k_{y}}\left\{A_{k_{y}} t_{\alpha}^{\dagger}\left(k_{y}\right) t_{\alpha}\left(k_{y}\right)\right. \\
\left.\quad+B_{k_{y}}\left(t_{\alpha}^{\dagger}\left(k_{y}\right) t_{\alpha}^{\dagger}\left(-k_{y}\right)+t_{\alpha}\left(k_{y}\right) t_{\alpha}\left(-k_{y}\right)\right)\right\} \\
+\sum_{k_{y}}\left\{\epsilon_{+}\left(k_{y}\right) h_{+a}^{\dagger}\left(k_{y}\right) h_{+a}\left(k_{y}\right)\right. \\
\left.-D\left(k_{y}\right)\left(h_{+\uparrow}^{\dagger}\left(k_{y}\right) h_{+\downarrow}^{\dagger}\left(-k_{y}\right)+h_{+\downarrow}\left(-k_{y}\right) h_{+\uparrow}\left(k_{y}\right)\right)\right\} \\
+\sum_{k_{y}}\left\{\epsilon_{-}\left(k_{y}\right) h_{-a}^{\dagger}\left(k_{y}\right) h_{-a}\left(k_{y}\right)\right. \\
\left.+D\left(k_{y}\right)\left(h_{-\uparrow}^{\dagger}\left(k_{y}\right) h_{-\downarrow}^{\dagger}\left(-k_{y}\right)+h_{-\downarrow}\left(-k_{y}\right) h_{-\uparrow}\left(k_{y}\right)\right)\right\}
\end{aligned}
$$

where

$$
\epsilon_{0}=-\frac{3}{4} J \bar{s}^{2}-\mu\left(\bar{s}^{2}-\bar{d}^{2}+2 x-1\right)
$$

$$
\begin{aligned}
& -\xi\left(2 \bar{d}^{2}-2 x\right) \\
A_{k_{y}} & =\frac{J}{4}-\mu+\lambda J \bar{s}^{2} \cos k_{y} \\
B_{k_{y}} & =\frac{\lambda J}{2} \bar{s}^{2} \cos k_{y} \\
\epsilon_{+}\left(k_{y}\right) & =t\left(\bar{s}^{2}-2 \bar{d}^{2}\right) \cos k_{y}-t-\xi \\
\epsilon_{-}\left(k_{y}\right) & =t\left(\bar{s}^{2}-2 \bar{d}^{2}\right) \cos k_{y}+t-\xi \\
D\left(k_{y}\right) & =2 \sqrt{2} t \bar{d} \bar{s} \cos k_{y}
\end{aligned}
$$

The above Hamiltonian can be diagonalized by using Bogoliubov transformation. That is,

$$
\begin{aligned}
\gamma_{\alpha}\left(k_{y}\right) & =u_{t}\left(k_{y}\right) t_{\alpha}\left(k_{y}\right)+v_{t}\left(k_{y}\right) t_{\alpha}^{\dagger}\left(-k_{y}\right) \\
\beta_{ \pm a}\left(k_{y}\right) & =u_{ \pm}\left(k_{y}\right) h_{ \pm a}\left(k_{y}\right)+v_{ \pm}\left(k_{y}\right) \varepsilon_{a b} h_{ \pm b}^{\dagger}\left(-k_{y}\right)
\end{aligned}
$$

where

$$
\begin{aligned}
u_{t}^{2}\left(k_{y}\right) & =\frac{1}{2}\left(\frac{A_{k_{y}}}{\omega_{k_{y}}}+1\right) \\
v_{t}^{2}\left(k_{y}\right) & =\frac{1}{2}\left(\frac{A_{k_{y}}}{\omega_{k_{y}}}-1\right) \\
u_{t}\left(k_{y}\right) v_{t}\left(k_{y}\right) & =\frac{B_{k_{y}}}{\omega_{k_{y}}} \\
\omega_{k_{y}} & =\sqrt{A_{k_{y}}^{2}-4 B_{k_{y}}^{2}}
\end{aligned}
$$

and

$$
\begin{aligned}
u_{ \pm}^{2}\left(k_{y}\right) & =\frac{1}{2}\left(1+\frac{\epsilon_{ \pm}\left(k_{y}\right)}{\Omega_{ \pm}\left(k_{y}\right)}\right) \\
v_{ \pm}^{2}\left(k_{y}\right) & =\frac{1}{2}\left(1-\frac{\epsilon_{ \pm}\left(k_{y}\right)}{\Omega_{ \pm}\left(k_{y}\right)}\right) \\
u_{ \pm}\left(k_{y}\right) v_{ \pm}\left(k_{y}\right) & =\mp \frac{D\left(k_{y}\right)}{\Omega_{ \pm}\left(k_{y}\right)} \\
\Omega_{ \pm}\left(k_{y}\right) & =\sqrt{\epsilon_{ \pm}^{2}\left(k_{y}\right)+D^{2}\left(k_{y}\right)}
\end{aligned}
$$

In terms of Bogoliubov variables, Hamiltonian is written as follows.

$$
\begin{aligned}
& H=N \epsilon_{0}+\sum_{k_{y}} \omega_{k_{y}} \gamma_{\alpha}^{\dagger}\left(k_{y}\right) \gamma_{\alpha}\left(k_{y}\right)+\frac{3}{2} \sum_{k_{y}}\left(\omega_{k_{y}}-A_{k_{y}}\right) \\
& +\sum_{k_{y}} \Omega_{+}\left(k_{y}\right) \beta_{+a}^{\dagger}\left(k_{y}\right) \beta_{+a}\left(k_{y}\right) \\
& +\sum_{k_{y}}\left\{\epsilon_{+}\left(k_{y}\right)-\sqrt{\epsilon_{+}^{2}\left(k_{y}\right)+D^{2}\left(k_{y}\right)}\right\} \\
& +\sum_{k_{y}} \Omega_{-}\left(k_{y}\right) \beta_{-a}^{\dagger}\left(k_{y}\right) \beta_{-a}\left(k_{y}\right) \\
& +\sum_{k_{y}}\left\{\epsilon_{-}\left(k_{y}\right)-\sqrt{\epsilon_{-}^{2}\left(k_{y}\right)+D^{2}\left(k_{y}\right)}\right\}
\end{aligned}
$$




\section{a. Ground State Energy and Saddle-Point Equations}

Now the ground state energy per particle is given by:

$$
\begin{aligned}
\epsilon_{g r}= & \frac{\langle H\rangle_{g r}}{N} \\
= & \epsilon_{0}+\frac{3}{2} \int_{0}^{\pi} \frac{d k_{y}}{\pi}\left(\omega_{k_{y}}-A_{k_{y}}\right) \\
& +\int_{0}^{\pi} \frac{d k_{y}}{\pi}\left\{\epsilon_{+}\left(k_{y}\right)-\Omega_{+}\left(k_{y}\right)\right\} \\
& +\int_{0}^{\pi} \frac{d k_{y}}{\pi}\left\{\epsilon_{-}\left(k_{y}\right)-\Omega_{-}\left(k_{y}\right)\right\} \\
= & \epsilon_{0}^{\prime}+\frac{3}{2} \int_{0}^{\pi} \frac{d k_{y}}{\pi} \omega_{k_{y}} \\
& -\int_{0}^{\pi} \frac{d k_{y}}{\pi} \Omega_{+}\left(k_{y}\right)-\int_{0}^{\pi} \frac{d k_{y}}{\pi} \Omega_{-}\left(k_{y}\right)
\end{aligned}
$$

where

$$
\epsilon_{0}^{\prime}=-\frac{3}{4} J \bar{s}^{2}-\mu\left(\bar{s}^{2}-\bar{d}^{2}+2 x-\frac{5}{2}\right)-\xi\left(2+2 \bar{d}^{2}-2 x\right) .
$$

Note that constants obtained from integrating $A_{k_{y}}$ and $\epsilon_{ \pm}\left(k_{y}\right)$ are ignored.

The term contributed from the triplet bosons $\left(t_{\alpha}\right)$ can be intergrated explicitly by using the elliptic function $\mathbf{E}$.

$$
\begin{aligned}
\epsilon_{g r} & =\epsilon_{0}^{\prime}+\frac{3}{\pi} J\left(\frac{1}{4}-\frac{\mu}{J}\right) \sqrt{1+\eta} \mathbf{E}\left(\sqrt{\frac{2 \eta}{1+\eta}}\right) \\
& -\int_{0}^{\pi} \frac{d k_{y}}{\pi} \Omega_{+}\left(k_{y}\right)-\int_{0}^{\pi} \frac{d k_{y}}{\pi} \Omega_{-}\left(k_{y}\right)
\end{aligned}
$$

where

$$
\eta=\frac{2 \lambda \bar{s}^{2}}{\frac{1}{4}-\frac{\mu}{J}} .
$$

Parameters in the Hamiltonian are determined by the saddle point condition in the mean-field approximation. In other words,

$$
\frac{\partial \epsilon_{g r}}{\partial \bar{s}}=\frac{\partial \epsilon_{g r}}{\partial \mu}=\frac{\partial \epsilon_{g r}}{\partial \bar{d}}=\frac{\partial \epsilon_{g r}}{\partial \xi}=0 .
$$

Explicit saddle-point equations are written as follows.

$$
\begin{aligned}
\frac{\partial\left(\epsilon_{g r} / J\right)}{\partial \bar{s}^{2}} & =-\frac{3}{4}-\frac{\mu}{J}+\frac{3 \lambda}{\pi \eta}\left\{\sqrt{1+\eta} \mathbf{E}\left(\sqrt{\frac{2 \eta}{1+\eta}}\right)\right. \\
& \left.-\frac{1}{\sqrt{1+\eta}} \mathbf{K}\left(\sqrt{\frac{2 \eta}{1+\eta}}\right)\right\} \\
& -\frac{t / J}{\bar{s}} \int_{0}^{\pi} \frac{d k_{y}}{\pi} \cos k_{y}\left\{\frac{\bar{s} \epsilon_{+}\left(k_{y}\right)+\sqrt{2} \bar{d} D\left(k_{y}\right)}{\Omega_{+}\left(k_{y}\right)}\right. \\
& \left.+\frac{\bar{s} \epsilon_{-}\left(k_{y}\right)+\sqrt{2} \bar{d} D\left(k_{y}\right)}{\Omega_{-}\left(k_{y}\right)}\right\}=0
\end{aligned}
$$

$$
\begin{aligned}
\frac{\partial\left(\epsilon_{g r} / J\right)}{\partial(\mu / J)} & =\bar{d}^{2}-\bar{s}^{2}+\frac{5}{2}-2 x \\
& -\frac{3}{2 \pi}\left\{\sqrt{1+\eta} \mathbf{E}\left(\sqrt{\frac{2 \eta}{1+\eta}}\right)\right. \\
& \left.+\frac{1}{\sqrt{1+\eta}} \mathbf{K}\left(\sqrt{\frac{2 \eta}{1+\eta}}\right)\right\}=0 \\
\frac{\partial\left(\epsilon_{g r} / J\right)}{\partial \bar{d}^{2}} & =\frac{\mu}{J}-2 \frac{t}{J} \frac{\xi}{t} \quad(\mathrm{~B} 42) \\
& +\frac{t / J}{\bar{d}} \int_{0}^{\pi} \frac{d k_{y}}{\pi} \cos k_{y}\left\{\frac{2 \bar{d} \epsilon_{+}\left(k_{y}\right)-\sqrt{2} \bar{s} D\left(k_{y}\right)}{\Omega_{+}\left(k_{y}\right)}\right. \\
& \left.+\frac{2 \bar{d} \epsilon_{-}\left(k_{y}\right)-\sqrt{2} \bar{s} D\left(k_{y}\right)}{\Omega_{-}\left(k_{y}\right)}\right\}=0 \\
\frac{\partial\left(\epsilon_{g r} / J\right)}{\partial(\xi / J)} & =-2-2 \bar{d}^{2}+2 x \\
& +\int_{0}^{\pi} \frac{d k_{y}}{\pi}\left\{\frac{\epsilon_{+}\left(k_{y}\right)}{\Omega_{+}\left(k_{y}\right)}+\frac{\epsilon_{-}\left(k_{y}\right)}{\Omega_{-}\left(k_{y}\right)}\right\}=0
\end{aligned}
$$

$$
\text { b. Limiting Case with } \bar{d}=0
$$

The above saddle-point equations are highly non-linear as a function of parameters $\bar{s}, \mu, \bar{d}$, and $\xi$. Since analytic solution is not accessible in this case, it is natural to make use of a numerical method. In general, solving a set of non-linear equations numerically is quite sensitive to the initial guesses of parameters. Therefore, it is very desirable to have some limiting situation where an explicit, analytic solution is available. Such a situation is realized when we set $\bar{d}=0$, i.e. turning off the pairing terms. Then, the ground state energy is given as follows.

$$
\begin{aligned}
\epsilon_{g r} & =\epsilon_{0}-\frac{3}{2} J\left(\frac{1}{4}-\frac{\mu}{J}\right)\left\{1-\frac{2}{\pi} \sqrt{1+\eta} \mathbf{E}\left(\sqrt{\frac{2 \eta}{1+\eta}}\right)\right\} \\
& +\int_{0}^{\pi} \frac{d k_{y}}{\pi}\left\{\epsilon_{+}\left(k_{y}\right)-\Omega_{+}\left(k_{y}\right)\right\}
\end{aligned}
$$

where the contribution from the anti-bonding fermion $\left(h_{-}\right)$is ignored because, in the case of $t / J \geq 1$, the anti-bonding fermion will have a reasonably larger energy than the bonding one, separated by roughly $2 t$. And therefore its contribution to the ground state energy is very small.

The fermion contribution can be simplified further.

$$
\begin{aligned}
\int_{0}^{\pi} \frac{d k_{y}}{\pi}\left\{\epsilon_{+}\left(k_{y}\right)\right. & \left.-\Omega_{+}\left(k_{y}\right)\right\} \\
& =\frac{2}{\pi} \int_{k_{F}}^{\pi} d k_{y}\left[\epsilon_{+}\left(k_{y}\right)\right]_{\bar{d}=0} \\
& =\frac{2}{\pi} \int_{k_{F}}^{\pi} d k_{y}\left(t \bar{s}^{2} \cos k_{y}-t-\xi\right) \\
& =-\frac{2}{\pi}\left\{(t+\xi)\left(\pi-k_{F}\right)+t \bar{s}^{2} \sin k_{F}\right\}
\end{aligned}
$$




$$
=\frac{2}{\pi} t \bar{s}^{2}\{\pi x \cos (\pi x)-\sin (\pi x)\}
$$

where the chemical potential $\xi$ and the Fermi wavevector $k_{F}$ are determined by:

$$
\begin{aligned}
\sum_{k_{y}} h_{+a}^{\dagger}\left(k_{y}\right) h_{+a}\left(k_{y}\right) & =x \Leftrightarrow \frac{2}{\pi}\left(\pi-k_{F}\right)=2 x \\
\epsilon_{+}\left(k_{F}\right) & =0 \Leftrightarrow \xi=t \bar{s}^{2} \cos k_{F}-t .
\end{aligned}
$$

Now the saddle-point equations are reduced to the following equations.

$$
\begin{aligned}
\frac{\partial\left(\epsilon_{g r} / J\right)}{\partial \bar{s}^{2}} & =-\left(\frac{3}{4}+\frac{\mu}{J}\right)+\frac{3 \lambda}{\pi \eta}\left\{\sqrt{1+\eta} \mathbf{E}\left(\sqrt{\frac{2 \eta}{1+\eta}}\right)\right. \\
& \left.-\frac{1}{\sqrt{1+\eta}} \mathbf{K}\left(\sqrt{\frac{2 \eta}{1+\eta}}\right)\right\} \\
& +\frac{2 t}{\pi}(\pi x \cos (\pi x)-\sin (\pi x))=0 \\
\frac{\partial\left(\epsilon_{g r} / J\right)}{\partial(\mu / J)} & =\frac{5}{2}-2 x-\bar{s}^{2}-\frac{3}{2 \pi}\left\{\sqrt{1+\eta} \mathbf{E}\left(\sqrt{\frac{2 \eta}{1+\eta}}\right)\right. \\
& \left.+\frac{1}{\sqrt{1+\eta}} \mathbf{K}\left(\sqrt{\frac{2 \eta}{1+\eta}}\right)\right\}=0
\end{aligned}
$$

Note that the other saddle-point equations are explicitly solved. In other words,

$$
\begin{aligned}
& \bar{d}=0 \\
& \xi=-t \bar{s}^{2} \cos (\pi x)-t
\end{aligned}
$$

Convenient simplification is realized from the fact that

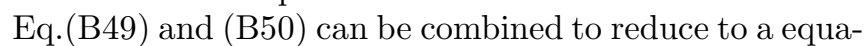
tion containing only one parameter $\eta$. That is,

$$
\begin{array}{r}
\frac{\eta}{2 \lambda}\left\{1-\frac{2}{\pi} \frac{t}{J}(\pi x \cos (\pi x)-\sin (\pi x))\right\} \\
=\frac{5}{2}-2 x-\frac{3}{\pi} \frac{1}{\sqrt{1+\eta}} \mathbf{K}\left(\sqrt{\frac{2 \eta}{1+\eta}}\right)
\end{array}
$$

where, once again, $\eta=\frac{2 \lambda \bar{s}^{2}}{1 / 4-\mu / J}$.

\section{Mean-Field Hamiltonian with Quartic Terms}

In this section, effects of quartic terms such as $H_{t^{4}}$, $H_{h^{4}}$, and $H_{t^{2} h^{2}}$ are included approximately by using quadratic decoupling. Let us start from $H_{t^{4}}$.

$$
\begin{aligned}
H_{t^{4}} & =-\frac{\lambda J}{2} \sum_{\langle i, j\rangle} \epsilon_{\alpha \beta \gamma} \epsilon_{\alpha \mu \nu} t_{i \beta}^{\dagger} t_{i \gamma} t_{j \mu}^{\dagger} t_{j \nu} \\
& =-\frac{\lambda J}{2} \sum_{\langle i, j\rangle}\left\{t_{i \alpha}^{\dagger} t_{i \beta} t_{j \alpha}^{\dagger} t_{j \beta}-t_{i \alpha}^{\dagger} t_{i \beta} t_{j \beta}^{\dagger} t_{j \alpha}\right\}
\end{aligned}
$$

Quadratic decoupling is carried out as follows.

$$
\begin{aligned}
t_{i \alpha}^{\dagger} t_{j \alpha}^{\dagger} t_{i \beta} t_{j \beta} & \simeq\left\langle t_{i \alpha}^{\dagger} t_{j \alpha}^{\dagger}\right\rangle t_{i \beta} t_{j \beta}+t_{i \alpha}^{\dagger} t_{j \alpha}^{\dagger}\left\langle t_{i \beta} t_{j \beta}\right\rangle \\
& -\left\langle t_{i \alpha}^{\dagger} t_{j \alpha}^{\dagger}\right\rangle\left\langle t_{i \beta} t_{j \beta}\right\rangle \\
t_{i \alpha}^{\dagger} t_{j \beta}^{\dagger} t_{i \beta} t_{j \alpha} & \simeq\left\langle t_{i \alpha}^{\dagger} t_{j \alpha}\right\rangle t_{j \beta}^{\dagger} t_{i \beta}+t_{i \alpha}^{\dagger} t_{j \alpha}\left\langle t_{j \beta}^{\dagger} t_{i \beta}\right\rangle \\
& -\left\langle t_{i \alpha}^{\dagger} t_{j \alpha}\right\rangle\left\langle t_{j \beta}^{\dagger} t_{i \beta}\right\rangle
\end{aligned}
$$

Therefore, $H_{t^{4}}$ is given by:

$$
\begin{aligned}
H_{t^{4}} & =\frac{\lambda J}{2} \sum_{\langle i, j\rangle}\left\{P_{y}\left(t_{i \alpha}^{\dagger} t_{j \alpha}+t_{j \alpha}^{\dagger} t_{i \alpha}\right)\right. \\
& \left.-Q_{y}\left(t_{i \alpha}^{\dagger} t_{j \alpha}^{\dagger}+t_{j \alpha} t_{i \alpha}\right)\right\} \\
& -N \frac{\lambda J}{2}\left(P_{y}^{2}-Q_{y}^{2}\right) \\
& =\frac{\lambda J}{2} \sum_{k_{y}} \cos k_{y}\left\{2 P_{y} t_{\alpha}^{\dagger}\left(k_{y}\right) t_{\alpha}\left(k_{y}\right)\right. \\
& \left.-Q_{y}\left(t_{\alpha}^{\dagger}\left(k_{y}\right) t_{\alpha}^{\dagger}\left(-k_{y}\right)+t_{\alpha}\left(-k_{y}\right) t_{\alpha}\left(k_{y}\right)\right)\right\} \\
& -N \frac{\lambda J}{2}\left(P_{y}^{2}-Q_{y}^{2}\right)
\end{aligned}
$$

where

$$
\begin{aligned}
P_{y} & \equiv\left\langle t_{i \alpha}^{\dagger} t_{j \alpha}\right\rangle=\sum_{k_{y}} \cos k_{y}\left\langle t_{\alpha}^{\dagger}\left(k_{y}\right) t_{\alpha}\left(k_{y}\right)\right\rangle \\
Q_{y} & \equiv\left\langle t_{i \alpha}^{\dagger} t_{j \alpha}^{\dagger}\right\rangle=\sum_{k_{y}} \cos k_{y}\left\langle t_{\alpha}^{\dagger}\left(k_{y}\right) t_{\alpha}^{\dagger}\left(-k_{y}\right)\right\rangle .
\end{aligned}
$$

Note that the translational symmetry is assumed. Now let us turn to the Hamiltonian $H_{t^{2} h^{2}}$.

$$
\begin{aligned}
H_{t^{2} h^{2}} & =-\frac{t}{2} \varepsilon_{a c} \varepsilon_{a e} \sigma_{c b}^{\alpha} \sigma_{e d}^{\beta} \sum_{\langle i, j\rangle}\left\{t_{i \alpha}^{\dagger} t_{j \beta}\left(h_{1 i b} h_{1 j d}^{\dagger}+h_{2 i b} h_{2 j d}^{\dagger}\right)\right. \\
& +(\text { h.c. })\}
\end{aligned}
$$

Remembering that, in the present approximation, nonzero quadratic contraction is obtained only when $\alpha=\beta$ for $\left\langle t_{\alpha}^{\dagger} t_{\beta}\right\rangle$, and $a=b$ for $\left\langle h_{a}^{\dagger} h_{b}\right\rangle$, the Hamiltonian $H_{t^{2} h^{2}}$ is quadratically decoupled as follows.

$$
\begin{aligned}
+ & \varepsilon_{a c} \varepsilon_{a e} \sigma_{c b}^{\alpha} \bar{\sigma}_{e d}^{\beta} \sum_{\langle i, j\rangle} t_{i \alpha}^{\dagger} t_{j \beta}\left\{h_{1 i b} h_{1 j d}^{\dagger}+(h . c .)\right\} \\
\simeq & \varepsilon_{a c} \varepsilon_{a e} \sigma_{c b}^{\alpha} \bar{\sigma}_{e b}^{\alpha} \sum_{\langle i, j\rangle} t_{i \alpha}^{\dagger} t_{j \alpha}\left\{h_{1 i b} h_{1 j b}^{\dagger}+(h . c .)\right\} \\
\simeq & -\left\langle t_{i \alpha}^{\dagger} t_{j \alpha}\right\rangle h_{1 j a}^{\dagger} h_{1 i a}-t_{i \alpha}^{\dagger} t_{j \alpha}\left\langle h_{1 j a}^{\dagger} h_{1 i a}\right\rangle \\
& +\left\langle t_{i \alpha}^{\dagger} t_{j \alpha}\right\rangle\left\langle h_{1 j a}^{\dagger} h_{1 i a}\right\rangle+(h . c .) \\
= & -P_{y}\left(h_{1 j a}^{\dagger} h_{1 i a}+h_{1 i a}^{\dagger} h_{1 j a}\right)-\Pi_{y}\left(t_{i \alpha}^{\dagger} t_{j \alpha}+t_{i \alpha}^{\dagger} t_{j \alpha}\right) \\
& +2 P_{y} \Pi_{y}
\end{aligned}
$$

where

$$
\Pi_{y} \equiv\left\langle h_{1 j a}^{\dagger} h_{1 i a}\right\rangle=\sum_{k_{y}} \cos k_{y}\left\langle h_{1 a}^{\dagger}\left(k_{y}\right) h_{1 a}\left(k_{y}\right)\right\rangle .
$$


Once again it is convenient to use the bonding and antibonding fermion representation. By using the reflection symmetry, $\Pi_{y}$ is given by:

$$
\begin{aligned}
\Pi_{y}= & \sum_{k_{y}} \cos k_{y}\left\langle h_{1 a}^{\dagger}\left(k_{y}\right) h_{1 a}\left(k_{y}\right)\right\rangle \\
= & \sum_{k_{y}} \cos k_{y}\left\langle h_{2 a}^{\dagger}\left(k_{y}\right) h_{2 a}\left(k_{y}\right)\right\rangle \\
= & \frac{1}{2} \sum_{k_{y}} \cos k_{y}\left\{\left\langle h_{+a}^{\dagger}\left(k_{y}\right) h_{+a}\left(k_{y}\right)\right\rangle\right. \\
& \left.+\left\langle h_{-a}^{\dagger}\left(k_{y}\right) h_{-a}\left(k_{y}\right)\right\rangle\right\}
\end{aligned}
$$

Finally, the Hamiltonian $H_{t^{2} h^{2}}$ is given by:

$$
\begin{aligned}
H_{t^{2} h^{2}} & =t P_{y} \sum_{k_{y}} \cos k_{y}\left(h_{+a}^{\dagger}(k) h_{+a}(k)+h_{-a}^{\dagger}(k) h_{-a}(k)\right) \\
& +2 t \Pi_{y} \sum_{k_{y}} \cos k_{y} t_{\alpha}^{\dagger}\left(k_{y}\right) t_{\alpha}\left(k_{y}\right) \\
& -4 t N P_{y} \Pi_{y}
\end{aligned}
$$

Now, quadratic decoupling of $H_{h^{4}}$ is performed. First, $H_{h^{4}}$ is given by:

$$
\begin{aligned}
H_{h^{4}}= & \frac{\lambda J}{4} \sum_{\langle i, j,\rangle} \sigma_{a b}^{\alpha} \sigma_{c d}^{\alpha}\left\{h_{1 i a}^{\dagger} h_{1 i b} h_{1 j c}^{\dagger} h_{1 j d}\right. \\
& \left.+h_{2 i a}^{\dagger} h_{2 i b} h_{2 j c}^{\dagger} h_{2 j d}\right\}
\end{aligned}
$$

Suppressing the site index within a dimer, i.e. 1 or 2 , let us first consider the following term inside summation.

$$
\begin{aligned}
& \sigma_{a b}^{\alpha} \sigma_{c d}^{\alpha} h_{i a}^{\dagger} h_{i b} h_{j c}^{\dagger} h_{j d} \\
= & 2\left\{h_{i \uparrow}^{\dagger} h_{i \downarrow} h_{j \downarrow}^{\dagger} h_{j \uparrow}+h_{i \downarrow}^{\dagger} h_{i \uparrow} h_{j \uparrow}^{\dagger} h_{j \downarrow}\right\} \\
& +\left\{h_{i \uparrow}^{\dagger} h_{i \uparrow}-h_{i \downarrow}^{\dagger} h_{i \downarrow}\right\}\left\{h_{j \uparrow}^{\dagger} h_{j \uparrow}-h_{j \downarrow}^{\dagger} h_{j \downarrow}\right\} \\
= & -\frac{3}{2} \Pi_{y}\left(h_{j a}^{\dagger} h_{i a}+h_{i a}^{\dagger} h_{j a}\right)+\frac{3}{2} \Pi_{y}^{2} \\
- & 3 \Delta_{y}\left(h_{i \uparrow}^{\dagger} h_{j \downarrow}^{\dagger}+h_{j \downarrow} h_{i \uparrow}\right)-3 \Delta_{y}\left(h_{j \uparrow}^{\dagger} h_{i \downarrow}^{\dagger}+h_{i \downarrow} h_{j \uparrow}\right) \\
& +6 \Delta_{y}^{2}
\end{aligned}
$$

where, once again, $\Pi_{y}$ is given by:

$$
\begin{aligned}
\Pi_{y}= & \frac{1}{2} \sum_{k_{y}} \cos k_{y}\left\{\left\langle h_{+a}^{\dagger}\left(k_{y}\right) h_{+a}\left(k_{y}\right)\right\rangle\right. \\
& \left.+\left\langle h_{-a}^{\dagger}\left(k_{y}\right) h_{-a}\left(k_{y}\right)\right\rangle\right\},
\end{aligned}
$$

and also $\Delta_{y}$ is defined as follows:

$$
\begin{aligned}
\Delta_{y}= & \frac{1}{2} \sum_{k_{y}} \cos k_{y}\left\{\left\langle h_{+\uparrow}^{\dagger}\left(k_{y}\right) h_{+\downarrow}^{\dagger}\left(-k_{y}\right)\right\rangle\right. \\
& \left.+\left\langle h_{-\uparrow}^{\dagger}\left(k_{y}\right) h_{-\downarrow}^{\dagger}\left(-k_{y}\right)\right\rangle\right\} .
\end{aligned}
$$

Therefore $H_{h^{4}}$ can be written as follows.

$$
\begin{aligned}
H_{h^{4}}= & -\frac{3}{4} \lambda J \sum_{k_{y}} \cos k_{y}\left\{\Pi_{y} h_{+a}^{\dagger}\left(k_{y}\right) h_{+a}\left(k_{y}\right)\right. \\
& \left.+2 \Delta_{y}\left(h_{+\uparrow}^{\dagger}\left(k_{y}\right) h_{+\downarrow}^{\dagger}\left(-k_{y}\right)+h_{+\downarrow}\left(-k_{y}\right) h_{+\uparrow}\left(k_{y}\right)\right)\right\} \\
- & \frac{3}{4} \lambda J \sum_{k_{y}} \cos k_{y}\left\{\Pi_{y} h_{-a}^{\dagger}\left(k_{y}\right) h_{-a}\left(k_{y}\right)\right. \\
& \left.+2 \Delta_{y}\left(h_{-\uparrow}^{\dagger}\left(k_{y}\right) h_{-\downarrow}^{\dagger}\left(-k_{y}\right)+h_{-\downarrow}\left(-k_{y}\right) h_{-\uparrow}\left(k_{y}\right)\right)\right\} \\
+ & \frac{3}{4} N \lambda J\left(\Pi_{y}^{2}+4 \Delta_{y}^{2}\right)
\end{aligned}
$$

Including all the quartic terms, the full, mean-field Hamiltonian is finally given by:

$$
\begin{aligned}
H & =N \epsilon_{0}+\sum_{k_{y}}\left\{A_{k_{y}}^{\prime} t_{\alpha}^{\dagger}\left(k_{y}\right) t_{\alpha}\left(k_{y}\right)\right. \\
& \left.+B_{k_{y}}^{\prime}\left(t_{\alpha}^{\dagger}\left(k_{y}\right) t_{\alpha}^{\dagger}\left(-k_{y}\right)+t_{\alpha}\left(k_{y}\right) t_{\alpha}\left(-k_{y}\right)\right)\right\} \\
+ & \sum_{k_{y}}\left\{\epsilon_{+}^{\prime}\left(k_{y}\right) h_{+a}^{\dagger}\left(k_{y}\right) h_{+a}\left(k_{y}\right)\right. \\
& \left.-D_{+}^{\prime}\left(k_{y}\right)\left(h_{+\uparrow}^{\dagger}\left(k_{y}\right) h_{+\downarrow}^{\dagger}\left(-k_{y}\right)+h_{+\downarrow}\left(-k_{y}\right) h_{+\uparrow}\left(k_{y}\right)\right)\right\} \\
+ & \sum_{k_{y}}\left\{\epsilon_{-}^{\prime}\left(k_{y}\right) h_{-a}^{\dagger}\left(k_{y}\right) h_{-a}\left(k_{y}\right)\right. \\
& \left.+D_{-}^{\prime}\left(k_{y}\right)\left(h_{-\uparrow}^{\dagger}\left(k_{y}\right) h_{-\downarrow}^{\dagger}\left(-k_{y}\right)+h_{-\downarrow}\left(-k_{y}\right) h_{-\uparrow}\left(k_{y}\right)\right)\right\}
\end{aligned}
$$

where

$$
\begin{aligned}
\epsilon_{0}= & -\frac{3}{4} J \bar{s}^{2}-\mu\left(\bar{s}^{2}-\bar{d}^{2}+2 x-1\right) \\
& -\xi\left(2 \bar{d}^{2}-2 x\right) \\
A_{k_{y}}^{\prime}= & A_{k_{y}}+\lambda J P_{y} \cos k_{y}+2 t \Pi_{y} \cos k_{y} \\
= & \frac{J}{4}-\mu+\lambda J\left(\bar{s}^{2}+P_{y}\right) \cos k_{y}+2 t \Pi_{y} \cos k \\
B_{k_{y}}^{\prime}= & B_{k_{y}}-\frac{\lambda J}{2} Q_{y} \cos k_{y} \\
= & \frac{\lambda J}{2}\left(\bar{s}^{2}-Q_{y}\right) \cos k_{y} \\
\epsilon_{+}^{\prime}\left(k_{y}\right)= & \epsilon_{+}\left(k_{y}\right)-\frac{3}{4} \lambda J \Pi_{y} \cos k_{y}+t P_{y} \cos k_{y} \\
= & t\left(\bar{s}^{2}-2 \bar{d}^{2}+P_{y}\right) \cos k_{y}-\frac{3}{4} \lambda J \Pi_{y} \cos k_{y}-t-\xi \\
\epsilon_{-}^{\prime}\left(k_{y}\right)= & \epsilon_{-}\left(k_{y}\right)-\frac{3}{4} \lambda J \Pi_{y} \cos k_{y}+t P_{y} \cos k_{y} \\
= & t\left(\bar{s}^{2}-2 \bar{d}^{2}+P_{y}\right) \cos k_{y}-\frac{3}{4} \lambda J \Pi_{y} \cos k_{y}+t-\xi \\
D_{+}^{\prime}\left(k_{y}\right)= & D\left(k_{y}\right)+\frac{3}{2} \lambda J \Delta_{y} \cos k_{y}
\end{aligned}
$$




$$
\begin{aligned}
& =2 \sqrt{2} t \bar{d} \bar{s} \cos k_{y}+\frac{3}{2} \lambda J \Delta_{y} \cos k_{y} \\
D_{-}^{\prime}\left(k_{y}\right) & =D\left(k_{y}\right)-\frac{3}{2} \lambda J \Delta_{y} \cos k_{y} \\
& =2 \sqrt{2} t \bar{d} \bar{s} \cos k_{y}-\frac{3}{2} \lambda J \Delta_{y} \cos k_{y}
\end{aligned}
$$

Note that all the constants which are not explicitly dependent on $\bar{s}, \mu, \bar{d}$, and $\xi$ are ignored. As previously, the above Hamiltonian can be diagonalized by using Bogoliubov transformation. That is,

$$
\begin{aligned}
H= & N \epsilon_{0}+\sum_{k_{y}} \omega_{k_{y}}^{\prime} \gamma_{\alpha}^{\dagger}\left(k_{y}\right) \gamma_{\alpha}\left(k_{y}\right)+\frac{3}{2} \sum_{k_{y}}\left(\omega_{k_{y}}^{\prime}-A_{k_{y}}^{\prime}\right) \\
+ & \sum_{k_{y}} \Omega_{+}^{\prime}\left(k_{y}\right) \beta_{+a}^{\dagger}\left(k_{y}\right) \beta_{+a}\left(k_{y}\right) \\
& +\sum_{k_{y}}\left(\epsilon_{+}^{\prime}\left(k_{y}\right)-\Omega_{+}^{\prime}\left(k_{y}\right)\right) \\
+ & \sum_{k_{y}} \Omega_{-}^{\prime}\left(k_{y}\right) \beta_{-a}^{\dagger}\left(k_{y}\right) \beta_{-a}\left(k_{y}\right) \\
& +\sum_{k_{y}}\left(\epsilon_{-}^{\prime}\left(k_{y}\right)-\Omega_{-}^{\prime}\left(k_{y}\right)\right)
\end{aligned}
$$

Saddle-point equations of the full Hamiltonian with quartic terms are similar to those without quartic terms except the fact that even the $t$ boson part is not expressed explicitly.

$$
\begin{aligned}
\frac{\partial\left(\epsilon_{g r} / J\right)}{\partial \bar{s}^{2}}= & -\frac{3}{4}-\frac{\mu}{J}+\frac{3}{2} \lambda \int_{0}^{\pi} \frac{d k_{y}}{\pi} \cos k_{y} \frac{A_{k_{y}}^{\prime}-2 B_{k_{y}}^{\prime}}{\omega_{k_{y}}^{\prime}} \\
- & \frac{t / J}{\bar{s}} \int_{0}^{\pi} \frac{d k_{y}}{\pi} \cos k_{y}\left\{\frac{\bar{s} \epsilon_{+}^{\prime}\left(k_{y}\right)+\sqrt{2} \bar{d} D_{+}^{\prime}\left(k_{y}\right)}{\Omega_{+}^{\prime}\left(k_{y}\right)}\right. \\
& \left.+\frac{\bar{s} \epsilon_{-}^{\prime}\left(k_{y}\right)+\sqrt{2} \bar{d} D_{-}^{\prime}\left(k_{y}\right)}{\Omega_{-}^{\prime}\left(k_{y}\right)}\right\}=0 \\
\frac{\partial\left(\epsilon_{g r} / J\right)}{\partial(\mu / J)}= & \bar{d}^{2}-\bar{s}^{2}+\frac{5}{2}-2 x-\frac{3}{2} \int_{0}^{\pi} \frac{d k_{y}}{\pi} \frac{A_{k_{y}}^{\prime}}{\omega_{k_{y}}^{\prime}}=0 \\
\frac{\partial\left(\epsilon_{g r} / J\right)}{\partial \bar{d}^{2}}= & \frac{\mu}{J}-2 \frac{t}{J} \frac{\xi}{t} \\
+ & \frac{t / J}{\bar{d}} \int_{0}^{\pi} \frac{d k_{y}}{\pi} \cos k_{y}\left\{\frac{2 \bar{d} \epsilon_{+}^{\prime}\left(k_{y}\right)-\sqrt{2} \bar{s} D_{+}^{\prime}\left(k_{y}\right)}{\Omega_{+}^{\prime}\left(k_{y}\right)}\right. \\
& \left.+\frac{2 \bar{d} \epsilon_{-}^{\prime}\left(k_{y}\right)-\sqrt{2} \bar{s} D_{-}^{\prime}\left(k_{y}\right)}{\Omega_{-}^{\prime}\left(k_{y}\right)}\right\}=0 \\
\frac{\partial\left(\epsilon_{g r} / J\right)}{\partial(\xi / J)}= & -2-2 \bar{d}^{2}+2 x \\
& +\int_{0}^{\pi} \frac{d k_{y}}{\pi}\left\{\frac{\epsilon_{+}^{\prime}\left(k_{y}\right)}{\Omega_{+}^{\prime}\left(k_{y}\right)}+\frac{\epsilon_{-}^{\prime}\left(k_{y}\right)}{\Omega_{-}^{\prime}\left(k_{y}\right)}\right\}=0
\end{aligned}
$$

However, the above saddle-point equations are not meaningful without the knowledge of $P_{y}, Q_{y}, \Pi_{y}$, and
$\Delta_{y}$ used in $A_{k_{y}}^{\prime}, B_{k_{y}}^{\prime}, \epsilon_{ \pm}^{\prime}\left(k_{y}\right)$, and $D_{ \pm}^{\prime}\left(k_{y}\right)$. These are the formulas for them.

$$
\begin{aligned}
& P_{y}=\frac{3}{2} \int_{0}^{\pi} \frac{d k_{y}}{\pi} \cos k_{y} \frac{A_{k_{y}}^{\prime}}{\omega_{k_{y}}^{\prime}} \\
& Q_{y}=-3 \int_{0}^{\pi} \frac{d k_{y}}{\pi} \cos k_{y} \frac{B_{k_{y}}^{\prime}}{\omega_{k_{y}}^{\prime}} \\
& \Pi_{y}=-\frac{1}{2} \int_{0}^{\pi} \frac{d k_{y}}{\pi} \cos k_{y}\left\{\frac{\epsilon_{+}^{\prime}\left(k_{y}\right)}{\Omega_{+}^{\prime}\left(k_{y}\right)}+\frac{\epsilon_{-}^{\prime}\left(k_{y}\right)}{\Omega_{-}^{\prime}\left(k_{y}\right)}\right\} \\
& \Delta_{y}=\frac{1}{2} \int_{0}^{\pi} \frac{d k_{y}}{\pi} \cos k_{y}\left\{\frac{D_{+}^{\prime}\left(k_{y}\right)}{\Omega_{+}^{\prime}\left(k_{y}\right)}-\frac{D_{-}^{\prime}\left(k_{y}\right)}{\Omega_{-}^{\prime}\left(k_{y}\right)}\right\}
\end{aligned}
$$

Upon solving the saddle-point equations, it is convenient to view $P_{y}, Q_{y}, \Pi_{y}$, and $\Delta_{y}$ as unknown parameters similar to $\bar{s}, \bar{d}, \mu$, and $\xi$. Then, the original saddlepoint conditions are equivalent to solving four saddlepoint equations and four equations for $P_{y}, Q_{y}, \Pi_{y}$, and $\Delta_{y}$ simultaneously.

\section{APPENDIX C: COMPUTATIONS FOR THE SQUARE LATTICE}

This section is devoted to the two-dimensional array of coupled t-J ladders. Hamiltonian for the coupling between two-leg t-J ladders can be written as follows.

$$
\begin{aligned}
H_{\perp} & =-t^{\prime} \sum_{i} \sum_{\langle n, m\rangle}\left\{c_{2 i n a}^{\dagger} c_{1 i m a}+c_{1 i m a}^{\dagger} c_{2 i n a}\right\} \\
& +\lambda^{\prime} J \sum_{i} \sum_{\langle n, m\rangle} S_{1 i m}^{\alpha} S_{2 i n}^{\alpha}
\end{aligned}
$$

where the index $i$ indicates the $i$-th dimer within a given ladder and the indices $n(m \equiv n+1)$ indicate the $n$-th $(m$-th) ladder. Derivation of the mean-field Hamiltonian for the dynamics perpenticular to the ladder direction, is rather similar to that of the parallel direction. But caution should be used in keeping track of signs of various terms in Hamiltonian. Let us start from the hopping term between ladders.

$$
\begin{aligned}
& c_{2 i n a}^{\dagger} c_{1 i m a} \\
= & \left\{h_{2 i n a}^{\dagger} d_{i n}+\frac{1}{\sqrt{2}} \varepsilon_{a b} s_{i n}^{\dagger} h_{1 i n b}+\frac{1}{\sqrt{2}} \varepsilon_{a c} \sigma_{c b}^{\alpha} t_{i n \alpha}^{\dagger} h_{1 i n b}\right\} \\
\times & \left\{d_{i m}^{\dagger} h_{1 i m a}+\frac{1}{\sqrt{2}} \varepsilon_{a c} h_{2 i m c}^{\dagger} s_{i m}-\frac{1}{\sqrt{2}} \varepsilon_{a e} \bar{\sigma}_{e d}^{\beta} h_{2 i m d}^{\dagger} t_{i m \beta}\right\} \\
\Rightarrow & \bar{d}^{2} h_{2 i n a}^{\dagger} h_{1 i m a}-\frac{1}{2} \varepsilon_{a b} \varepsilon_{a c} \bar{s}^{2} h_{2 i m c}^{\dagger} h_{1 i n b} \\
+ & \frac{1}{\sqrt{2}} \bar{d} \bar{s} \varepsilon_{a c} h_{2 i n a}^{\dagger} h_{2 i m c}^{\dagger}+\frac{1}{\sqrt{2}} \bar{d} \bar{s} \varepsilon_{a b} h_{1 i n b} h_{1 i m a} \\
- & \frac{1}{2} \varepsilon_{a c} \varepsilon_{a e} \sigma_{c b}^{\alpha} \bar{\sigma}_{e d}^{\beta} t_{i n \alpha}^{\dagger} t_{i m \beta} h_{1 i n b} h_{2 i m d}^{\dagger}
\end{aligned}
$$


Also, assuming that there is no magnetic ordering, the product of spin operators is given by:

$$
\begin{aligned}
& S_{1 i m \alpha} S_{2 i n \alpha} \\
= & \tilde{S}_{1 i m \alpha} \tilde{S}_{2 i n \alpha}+\frac{1}{4} \sigma_{a b}^{\alpha} \sigma_{c d}^{\alpha} h_{1 i m a}^{\dagger} h_{1 i m b} h_{2 i n c}^{\dagger} h_{2 i n d} \\
= & -\frac{1}{4}\left(s_{i m}^{\dagger} t_{i m \alpha}+t_{i m \alpha}^{\dagger} s_{i m}-i \epsilon_{\alpha \beta \gamma} t_{i m \beta}^{\dagger} t_{i m \gamma}\right) \\
& \times\left(s_{i n}^{\dagger} t_{i n \alpha}+t_{i n \alpha}^{\dagger} s_{i n}+i \epsilon_{\alpha \mu \nu} t_{i n \mu}^{\dagger} t_{i n \nu}\right) \\
+ & \frac{1}{4} \sigma_{a b}^{\alpha} \sigma_{c d}^{\alpha} h_{1 i m a}^{\dagger} h_{1 i m b} h_{2 i n c}^{\dagger} h_{2 i n d} \\
\Rightarrow & -\frac{1}{4} \bar{s}^{2}\left(t_{i m \alpha}^{\dagger} t_{i n \alpha}+t_{i n \alpha}^{\dagger} t_{i m \alpha}+t_{i m \alpha}^{\dagger} t_{i n \alpha}^{\dagger}+t_{i m \alpha} t_{i n \alpha}\right) \\
& +\frac{1}{4}\left(t_{i m \alpha}^{\dagger} t_{i n \alpha} t_{i n \beta}^{\dagger} t_{i m \beta}-t_{i m \alpha}^{\dagger} t_{i n \alpha}^{\dagger} t_{i m \beta} t_{i n \beta}\right) \\
& +\frac{1}{4} \sigma_{a b}^{\alpha} \sigma_{c d}^{\alpha} h_{1 i m a}^{\dagger} h_{1 i m b} h_{2 i n c}^{\dagger} h_{2 i n d}
\end{aligned}
$$

Performing the quadratic decoupling and ignoring irrelevent constants, the Hamiltonian $H_{\perp}$ is writtten as follows. (Note that $k_{x}$ is the momentum associated with the perpendicular direction to ladders, while $k_{y}$ is with the parallel direction. Also, in laboratory unit, momentum in the parallel direction is given by $p_{y}=k_{y} / a$, and that in the perpendicular direction is given by $p_{x}=k_{x} / 2 a$ where $a$ is the lattice spacing and $-\pi<k_{x}, k_{y} \leq \pi$.)

$$
\begin{aligned}
& H_{\perp}=H_{\perp, h^{2}}+H_{\perp, t^{2} h^{2}}+H_{\perp, t^{2}}+H_{\perp, t^{4}}+H_{\perp, h^{4}} \\
& H_{\perp, h^{2}=} \frac{t^{\prime}}{2}\left(\bar{s}^{2}-2 \bar{d}^{2}\right) \sum_{\mathbf{k}} \cos k_{x}\left\{h_{+a}^{\dagger}(\mathbf{k}) h_{+a}(\mathbf{k})\right. \\
&\left.-h_{-a}^{\dagger}(\mathbf{k}) h_{-a}(\mathbf{k})\right\} \\
&-\sqrt{2} t^{\prime} \bar{d} \bar{s} \sum_{\mathbf{k}} \cos k_{x}\left\{h_{+\uparrow}^{\dagger}(\mathbf{k}) h_{+\downarrow}^{\dagger}(-\mathbf{k})\right. \\
&\left.+h_{+\downarrow}(-\mathbf{k}) h_{+\uparrow}(\mathbf{k})\right\} \\
&- \sqrt{2} t^{\prime} \bar{d} \bar{s} \sum_{\mathbf{k}} \cos k_{x}\left\{h_{-\uparrow}^{\dagger}(\mathbf{k}) h_{-\downarrow}^{\dagger}(-\mathbf{k})\right. \\
&\left.+h_{-\downarrow}(-\mathbf{k}) h_{-\uparrow}(\mathbf{k})\right\} \\
& H_{\perp, t^{2} h^{2}=}-\frac{t^{\prime}}{2} P_{x} \sum_{\mathbf{k}} \cos k_{x}\left\{h_{+a}^{\dagger}(\mathbf{k}) h_{+a}(\mathbf{k})\right. \\
&\left.-h_{-a}^{\dagger}(\mathbf{k}) h_{-a}(\mathbf{k})\right\} \\
&- t^{\prime} \Pi_{x} \sum_{\mathbf{k}} \cos k_{x} t_{\alpha}^{\dagger}(\mathbf{k}) t_{\alpha}(\mathbf{k}) \\
& H_{\perp, t^{2}}=-\frac{\lambda^{\prime} J}{4} \bar{s}^{2} \sum_{\mathbf{k}} \cos k_{x}\left\{2 t_{\alpha}^{\dagger}(\mathbf{k}) t_{\alpha}(\mathbf{k})\right. \\
&\left.+t_{\alpha}^{\dagger}(\mathbf{k}) t_{\alpha}^{\dagger}(-\mathbf{k})+t_{\alpha}(\mathbf{k}) t_{\alpha}(-\mathbf{k})\right\} \\
& \lambda_{\perp, t^{4}}= \frac{\lambda^{\prime} J}{4} \sum_{\mathbf{k}} \cos k_{x}\left\{2 P_{x} t_{\alpha}^{\dagger}(\mathbf{k}) t_{\alpha}(\mathbf{k})\right. \\
&\left.-Q_{x}\left(t_{\alpha}^{\dagger}(\mathbf{k}) t_{\alpha}^{\dagger}(-\mathbf{k})+t_{\alpha}(\mathbf{k}) t_{\alpha}(-\mathbf{k})\right)\right\}
\end{aligned}
$$

$$
\begin{aligned}
H_{\perp, h^{4}}= & -\frac{3}{8} \lambda^{\prime} J \Pi_{x} \sum_{\mathbf{k}} \cos k_{x}\left\{h_{+a}^{\dagger}(\mathbf{k}) h_{+a}(\mathbf{k})\right. \\
& \left.-h_{-a}^{\dagger}(\mathbf{k}) h_{-a}(\mathbf{k})\right\} \\
- & \frac{3}{4} \lambda^{\prime} J \Delta_{x} \sum_{\mathbf{k}} \cos k_{x}\left\{h_{+\uparrow}^{\dagger}(\mathbf{k}) h_{+\downarrow}^{\dagger}(-\mathbf{k})\right. \\
& \left.+h_{+\downarrow}(-\mathbf{k}) h_{+\uparrow}(\mathbf{k})\right\} \\
+ & \frac{3}{4} \lambda^{\prime} J \Delta_{x} \sum_{\mathbf{k}} \cos k_{x}\left\{h_{-\uparrow}^{\dagger}(\mathbf{k}) h_{-\downarrow}^{\dagger}(-\mathbf{k})\right. \\
& \left.+h_{-\downarrow}(-\mathbf{k}) h_{-\uparrow}(\mathbf{k})\right\}
\end{aligned}
$$

where

$$
\begin{aligned}
P_{x}= & \sum_{\mathbf{k}} \cos k_{x}\left\langle t_{\alpha}^{\dagger}(\mathbf{k}) t_{\alpha}(\mathbf{k})\right\rangle \\
Q_{x}= & \sum_{\mathbf{k}} \cos k_{x}\left\langle t_{\alpha}^{\dagger}(\mathbf{k}) t_{\alpha}^{\dagger}(-\mathbf{k})\right\rangle . \\
\Pi_{x}= & \frac{1}{2} \sum_{\mathbf{k}} \cos k_{x}\left\{\left\langle h_{+a}^{\dagger}(\mathbf{k}) h_{+a}(\mathbf{k})\right\rangle-\left\langle h_{-a}^{\dagger}(\mathbf{k}) h_{-a}(\mathbf{k})\right\rangle\right\} \\
\Delta_{x}= & \frac{1}{2} \sum_{\mathbf{k}} \cos k_{x}\left\{\left\langle h_{+\uparrow}^{\dagger}(\mathbf{k}) h_{+\downarrow}^{\dagger}(-\mathbf{k})\right\rangle\right. \\
& \left.-\left\langle h_{-\uparrow}^{\dagger}(\mathbf{k}) h_{-\downarrow}^{\dagger}(-\mathbf{k})\right\rangle\right\}
\end{aligned}
$$

where the bonding and anti-bonding fermions are assumed to be well separated in energy so that the mixing terms between them are ignored.

Combining $H_{\perp}$ with the Hamiltonian describing the dynamics inside ladder, the mean-field Hamiltonian for the two-dimensional array of coupled t-J ladders is obtained.

$$
\begin{aligned}
H & =N^{2} \epsilon_{0} / 2+\sum_{\mathbf{k}}\left\{\tilde{A}_{\mathbf{k}} t_{\alpha}^{\dagger}(\mathbf{k}) t_{\alpha}(\mathbf{k})\right. \\
& \left.+\tilde{B}_{\mathbf{k}}\left(t_{\alpha}^{\dagger}(\mathbf{k}) t_{\alpha}^{\dagger}(-\mathbf{k})+t_{\alpha}(\mathbf{k}) t_{\alpha}(-\mathbf{k})\right)\right\} \\
+ & \sum_{\mathbf{k}}\left\{\tilde{\epsilon}_{+}(\mathbf{k}) h_{+a}^{\dagger}(\mathbf{k}) h_{+a}(\mathbf{k})\right. \\
& \left.-\tilde{D}_{+}(\mathbf{k})\left(h_{+\uparrow}^{\dagger}(\mathbf{k}) h_{+\downarrow}^{\dagger}(-\mathbf{k})+h_{+\downarrow}(-\mathbf{k}) h_{+\uparrow}(\mathbf{k})\right)\right\} \\
+ & \sum_{\mathbf{k}}\left\{\tilde{\epsilon}_{-}(\mathbf{k}) h_{-a}^{\dagger}(\mathbf{k}) h_{-a}(\mathbf{k})\right. \\
& \left.+\tilde{D}_{-}(\mathbf{k})\left(h_{-\uparrow}^{\dagger}(\mathbf{k}) h_{-\downarrow}^{\dagger}(-\mathbf{k})+h_{-\downarrow}(-\mathbf{k}) h_{-\uparrow}(\mathbf{k})\right)\right\}
\end{aligned}
$$

where

$$
\begin{aligned}
\epsilon_{0}= & -\frac{3}{4} J \bar{s}^{2}-\mu\left(\bar{s}^{2}-\bar{d}^{2}+2 x-1\right) \\
& -\xi\left(2 \bar{d}^{2}-2 x\right) \\
\tilde{A}_{\mathbf{k}}= & \frac{J}{4}-\mu+\lambda J \bar{s}^{2}\left(\cos k_{y}-\frac{\lambda^{\prime}}{2 \lambda} \cos k_{x}\right) \\
+ & \lambda J\left(P_{y} \cos k_{y}+\frac{\lambda^{\prime}}{2 \lambda} P_{x} \cos k_{x}\right)
\end{aligned}
$$




$$
\begin{aligned}
& +2 t\left(\Pi_{y} \cos k_{y}-\frac{t^{\prime}}{2 t} \Pi_{x} \cos k_{x}\right) \\
\tilde{B}_{\mathbf{k}} & =\frac{\lambda J}{2} \bar{s}^{2}\left(\cos k_{y}-\frac{\lambda^{\prime}}{2 \lambda} \cos k_{x}\right) \\
& -\frac{\lambda J}{2}\left(Q_{y} \cos k_{y}+\frac{\lambda^{\prime}}{2 \lambda} Q_{x} \cos k_{x}\right) \\
\tilde{\epsilon}_{+}(\mathbf{k}) & =t\left(\bar{s}^{2}-2 \bar{d}^{2}\right)\left(\cos k_{y}+\frac{t^{\prime}}{2 t} \cos k_{x}\right)-t-\xi \\
& -\frac{3}{4} \lambda J\left(\Pi_{y} \cos k_{y}+\frac{\lambda^{\prime}}{2 \lambda} \Pi_{x} \cos k_{x}\right) \\
& +t\left(P_{y} \cos k_{y}-\frac{t^{\prime}}{2 t} P_{x} \cos k_{x}\right) \\
\tilde{\epsilon}_{-}(\mathbf{k}) & =t\left(\bar{s}^{2}-2 \bar{d}^{2}\right)\left(\cos k_{y}-\frac{t^{\prime}}{2 t} \cos k_{x}\right)+t-\xi \\
& -\frac{3}{4} \lambda J\left(\Pi_{y} \cos k_{y}-\frac{\lambda^{\prime}}{2 \lambda} \Pi_{x} \cos k_{x}\right) \\
\tilde{D}_{-}(\mathbf{k}) & =2 \sqrt{2} t \bar{d} \bar{s}\left(\cos k_{y}-\frac{t^{\prime}}{2 t} \cos k_{x}\right) \\
& +t\left(P_{y} \cos k_{y}+\frac{t^{\prime}}{2 t} P_{x} \cos k_{x}\right) \\
& +\frac{3}{2} \lambda J\left(\Delta_{y} \cos k_{y}+\frac{\lambda^{\prime}}{2 \lambda} \Delta_{x} \cos k_{x}\right) \\
\tilde{D}_{+}(\mathbf{k})= & 2 \sqrt{2} t \bar{d} \bar{s}\left(\cos k_{y}+\frac{t^{\prime}}{2 t} \cos k_{x}\right) \\
&
\end{aligned}
$$

Diagonalization via Bogoliubov transformation and the correspondent saddle-point equations are similar to those of single ladder case. That is to say,

$$
\begin{aligned}
\epsilon_{g r} & =\epsilon^{\prime}+\frac{3}{2} \iint \frac{d^{2} \mathbf{k}}{\pi^{2}} \tilde{\omega}(\mathbf{k}) \\
& -\iint \frac{d^{2} \mathbf{k}}{\pi^{2}} \tilde{\Omega}_{+}(\mathbf{k})-\iint \frac{d^{2} \mathbf{k}}{\pi^{2}} \tilde{\Omega}_{-}(\mathbf{k})
\end{aligned}
$$

where

$$
\begin{aligned}
\epsilon_{0}^{\prime} & =-\frac{3}{4} J \bar{s}^{2}-\mu\left(\bar{s}^{2}-\bar{d}^{2}+2 x-\frac{5}{2}\right) \\
& -\xi\left(2+2 \bar{d}^{2}-2 x\right) \\
\tilde{\omega}(\mathbf{k}) & =\sqrt{\tilde{A}_{\mathbf{k}}^{2}-4 \tilde{B}_{\mathbf{k}}^{2}} \\
\tilde{\Omega}_{ \pm}(\mathbf{k}) & =\sqrt{\tilde{\epsilon}_{ \pm}^{2}(\mathbf{k})+\tilde{D}_{ \pm}^{2}(\mathbf{k}) .}
\end{aligned}
$$

where the upper and lower limit of integral, $\pi$ and 0 , are not explicitly written. Saddle-point equations are given as follows.

$$
\begin{aligned}
& \frac{\partial\left(\epsilon_{g r} / J\right)}{\partial \bar{s}^{2}}=-\frac{3}{4}-\frac{\mu}{J} \\
& +\frac{3}{2} \lambda \iint \frac{d^{2} \mathbf{k}}{\pi^{2}}\left(\cos k_{y}-\frac{\lambda^{\prime}}{2 \lambda} \cos k_{x}\right) \\
& \times \frac{\tilde{A}_{\mathbf{k}}-2 \tilde{B}_{\mathbf{k}}}{\tilde{\omega}_{\mathbf{k}}} \\
& -\frac{t / J}{\bar{s}} \iint \frac{d^{2} \mathbf{k}}{\pi^{2}}\left(\cos k_{y}+\frac{t^{\prime}}{2 t} \cos k_{x}\right) \\
& \times \frac{\bar{s} \tilde{\epsilon}_{+}(\mathbf{k})+\sqrt{2} \bar{d} \tilde{D}_{+}(\mathbf{k})}{\tilde{\Omega}_{+}(\mathbf{k})} \\
& -\frac{t / J}{\bar{s}} \iint \frac{d^{2} \mathbf{k}}{\pi^{2}}\left(\cos k_{y}-\frac{t^{\prime}}{2 t} \cos k_{x}\right) \\
& \times \frac{\bar{s} \tilde{\epsilon}_{-}(\mathbf{k})+\sqrt{2} \bar{d} \tilde{D}_{-}(\mathbf{k})}{\tilde{\Omega}_{-}(\mathbf{k})}=0 \\
& \frac{\partial\left(\epsilon_{g r} / J\right)}{\partial(\mu / J)}=\bar{d}^{2}-\bar{s}^{2}+\frac{5}{2}-2 x \\
& -\frac{3}{2} \iint \frac{d^{2} \mathbf{k}}{\pi^{2}} \frac{\tilde{A}_{\mathbf{k}}}{\tilde{\omega}_{\mathbf{k}}}=0 \\
& \frac{\partial\left(\epsilon_{g r} / J\right)}{\partial \bar{d}^{2}}=\frac{\mu}{J}-2 \frac{t}{J} \frac{\xi}{t} \\
& +\frac{t / J}{\bar{d}} \iint \frac{d^{2} \mathbf{k}}{\pi^{2}}\left(\cos k_{y}+\frac{t^{\prime}}{2 t} \cos k_{x}\right) \\
& \times \frac{2 \bar{d} \tilde{\epsilon}_{+}(\mathbf{k})-\sqrt{2} \bar{s} \tilde{D}_{+}(\mathbf{k})}{\tilde{\Omega}_{+}(\mathbf{k})} \\
& +\frac{t / J}{\bar{d}} \iint \frac{d^{2} \mathbf{k}}{\pi^{2}}\left(\cos k_{y}-\frac{t^{\prime}}{2 t} \cos k_{x}\right) \\
& \times \frac{2 \bar{d} \tilde{\epsilon}_{-}(\mathbf{k})-\sqrt{2} \bar{s} \tilde{D}_{-}(\mathbf{k})}{\tilde{\Omega}_{-}(\mathbf{k})}=0 \\
& \frac{\partial\left(\epsilon_{g r} / J\right)}{\partial(\xi / J)}=-2-2 \bar{d}^{2}+2 x \\
& +\iint \frac{d^{2} \mathbf{k}}{\pi^{2}}\left\{\frac{\tilde{\epsilon}_{+}(\mathbf{k})}{\tilde{\Omega}_{+}(\mathbf{k})}+\frac{\tilde{\epsilon}_{-}(\mathbf{k})}{\tilde{\Omega}_{-}(\mathbf{k})}\right\}=0
\end{aligned}
$$

Also, the parameters due to quartic terms are defined as follows.

$$
\begin{aligned}
& P_{y}=\frac{3}{2} \iint \frac{d^{2} \mathbf{k}}{\pi^{2}} \cos k_{y} \frac{\tilde{A}_{\mathbf{k}}}{\tilde{\omega}_{\mathbf{k}}} \\
& Q_{y}=-3 \iint \frac{d^{2} \mathbf{k}}{\pi^{2}} \cos k_{y} \frac{\tilde{B}_{\mathbf{k}}}{\tilde{\omega}_{\mathbf{k}}} \\
& \Pi_{y}=-\frac{1}{2} \iint \frac{d^{2} \mathbf{k}}{\pi^{2}} \cos k_{y}\left\{\frac{\tilde{\epsilon}_{+}(\mathbf{k})}{\tilde{\Omega}_{+}(\mathbf{k})}+\frac{\tilde{\epsilon}_{-}(\mathbf{k})}{\tilde{\Omega}_{-}(\mathbf{k})}\right\} \\
& \Delta_{y}=\frac{1}{2} \iint \frac{d^{2} \mathbf{k}}{\pi^{2}} \cos k_{y}\left\{\frac{\tilde{D}_{+}(\mathbf{k})}{\tilde{\Omega}_{+}(\mathbf{k})}-\frac{\tilde{D}_{-}(\mathbf{k})}{\tilde{\Omega}_{-}(\mathbf{k})}\right\}
\end{aligned}
$$




$$
\begin{aligned}
& P_{x}=\frac{3}{2} \iint \frac{d^{2} \mathbf{k}}{\pi^{2}} \cos k_{x} \frac{\tilde{A}_{\mathbf{k}}}{\tilde{\omega}_{\mathbf{k}}} \\
& Q_{x}=-3 \iint \frac{d^{2} \mathbf{k}}{\pi^{2}} \cos k_{x} \frac{\tilde{B}_{\mathbf{k}}}{\tilde{\omega}_{\mathbf{k}}} \\
& \Pi_{x}=-\frac{1}{2} \iint \frac{d^{2} \mathbf{k}}{\pi^{2}} \cos k_{x}\left\{\frac{\tilde{\epsilon}_{+}(\mathbf{k})}{\tilde{\Omega}_{+}(\mathbf{k})}-\frac{\tilde{\epsilon}_{-}(\mathbf{k})}{\tilde{\Omega}_{-}(\mathbf{k})}\right\} \\
& \Delta_{x}=\frac{1}{2} \iint \frac{d^{2} \mathbf{k}}{\pi^{2}} \cos k_{x}\left\{\frac{\tilde{D}_{+}(\mathbf{k})}{\tilde{\Omega}_{+}(\mathbf{k})}+\frac{\tilde{D}_{-}(\mathbf{k})}{\tilde{\Omega}_{-}(\mathbf{k})}\right\}
\end{aligned}
$$

As previously, it is convenient to have some limiting case when the explicit solution is available. We will take the case of $x=0$, i.e. no-doping case.

$$
\begin{aligned}
& \frac{\partial\left(\epsilon_{g r} / J\right)}{\partial \bar{s}^{2}}=-\frac{3}{4}-\frac{\mu}{J}+\frac{3}{2} \lambda \iint \frac{d^{2} \mathbf{k}}{\pi^{2}} \frac{f_{\mathbf{k}}}{\sqrt{1+\eta f_{\mathbf{k}}}}=0 \\
& \frac{\partial\left(\epsilon_{g r} / J\right)}{\partial(\mu / J)}=\frac{5}{2}-\bar{s}^{2}-\frac{3}{2} \iint \frac{d^{2} \mathbf{k}}{\pi^{2}} \frac{1+\frac{\eta}{2} f_{\mathbf{k}}}{\sqrt{1+\eta f_{\mathbf{k}}}}=0
\end{aligned}
$$

where $\eta=\frac{2 \lambda \bar{s}^{2}}{1 / 4-\mu / J}$ and $f_{\mathbf{k}}=\cos k_{y}-\frac{\lambda^{\prime}}{2 \lambda} \cos k_{x}$. As before, it is possible to combine the above two equations to get a single equation only for $\eta$. That is to say,

$$
\frac{\eta}{2 \lambda}=\frac{5}{2}-\frac{3}{2} \iint \frac{d^{2} \mathbf{k}}{\pi^{2}} \frac{1}{\sqrt{1+\eta f_{\mathbf{k}}}}
$$

\section{Superconducting Order Parameters}

This subsection relates the parameters in the above computations to the physical order parameters describing the nature of the superconducting state. In the case of two-dimensional array of coupled ladders, there are three order parameters to be defined:

$$
\begin{aligned}
\chi_{0} & \equiv \varepsilon_{a b}\left\langle c_{1 \text { ina }}^{\dagger} c_{2 i n b}^{\dagger}\right\rangle \\
\chi_{\|} & \equiv \varepsilon_{a b}\left\langle c_{1, i+1, n a}^{\dagger} c_{1 \text { inb }}^{\dagger}\right\rangle \\
\chi_{\perp} & \equiv \varepsilon_{a b}\left\langle c_{1 i, n+1, a}^{\dagger} c_{2 i n b}^{\dagger}\right\rangle
\end{aligned}
$$

The first one, $\chi_{0}$, is the pairing order parameter within dimer. Using constraints, it is written as follows.

$$
\begin{aligned}
\chi_{0} & =\varepsilon_{a b}\left\langle\left\{h_{1 i n a}^{\dagger} d_{i n}+\frac{1}{\sqrt{2}} \varepsilon_{a c} s_{i n}^{\dagger} h_{2 i n c}-\frac{1}{\sqrt{2}} \varepsilon_{a c} \sigma_{c d}^{\alpha} t_{i n \alpha}^{\dagger} h_{2 i n d}\right\}\right. \\
& \left.\times\left\{h_{2 i n b}^{\dagger} d_{i n}+\frac{1}{\sqrt{2}} \varepsilon_{b c} s_{i n}^{\dagger} h_{1 i n c}+\frac{1}{\sqrt{2}} \varepsilon_{b c} \bar{\sigma}_{c d}^{\beta} t_{i n \beta}^{\dagger} h_{1 i n d}\right\}\right\rangle \\
& =\varepsilon_{a b} \cdot \frac{1}{\sqrt{2}}\left\langle\varepsilon_{a c} s_{i n}^{\dagger} d_{i n} h_{2 i n c} h_{2 i n b}^{\dagger}\right\rangle=\sqrt{2} \bar{s} \bar{d}
\end{aligned}
$$

The pairing order parameter in the parallel direction, $\chi_{\|}$, is evaluated as follows.

$\chi_{\|}=\varepsilon_{a b}\left\{\bar{d}^{2}\left\langle h_{1, i+1, n a}^{\dagger} h_{1 i n b}^{\dagger}\right\rangle+\frac{1}{2} \varepsilon_{a c} \varepsilon_{b d} \bar{s}^{2}\left\langle h_{2, i+1, n a} h_{2 i n d}\right\rangle\right.$

$$
\begin{aligned}
& +\frac{1}{\sqrt{2}} \varepsilon_{b c} \bar{d} \bar{s}\left\langle h_{1, i+1, n a}^{\dagger} h_{2 i n c}\right\rangle+\frac{1}{\sqrt{2}} \varepsilon_{a c} \bar{d} \bar{s}\left\langle h_{2, i+1, n c} h_{1 i n b}^{\dagger}\right\rangle \\
& \left.+\frac{1}{2} \varepsilon_{a c} \varepsilon_{b e} \sigma_{c d}^{\alpha} \sigma_{e f}^{\beta}\left\langle t_{i+1, n \alpha}^{\dagger} t_{i n \beta}^{\dagger} h_{2, i+1, n d} h_{2 i n f}\right\rangle\right\} \\
& =\left(\bar{d}^{2}-\frac{1}{2} \bar{s}^{2}+Q\right) \Gamma-\sqrt{2} \bar{d} \bar{s} \tilde{\Pi}
\end{aligned}
$$

where $Q$ and $\Gamma$ are defined previously, and $\tilde{\Pi}$ is given by:

$$
\tilde{\Pi}=-\frac{1}{2} \iint \frac{d k d q}{\pi^{2}} \cos k\left\{\frac{\tilde{\epsilon}_{+}(k, q)}{\tilde{\Omega}_{+}(k, q)}-\frac{\tilde{\epsilon}_{-}(k, q)}{\tilde{\Omega}_{-}(k, q)}\right\} .
$$

Similarly, pairing order parameter in the perpendicular direction, $\chi_{\perp}$, is computed to give the following formula.

$$
\begin{aligned}
\chi_{\perp} & =\varepsilon_{a b}\left\{\bar{d}^{2}\left\langle h_{2 i n a}^{\dagger} h_{1 i, n+1, b}^{\dagger}\right\rangle+\frac{1}{2} \varepsilon_{a c} \varepsilon_{b d} \bar{s}^{2}\left\langle h_{1 i n c} h_{2 i, n+1, d}\right\rangle\right. \\
& +\frac{1}{\sqrt{2}} \varepsilon_{b c} \bar{d} \bar{s}\left\langle h_{2 i n a}^{\dagger} h_{2 i, n+1, c}\right\rangle+\frac{1}{\sqrt{2}} \varepsilon_{a c} \bar{d} \bar{s}\left\langle h_{1 i n c} h_{1 i, n+1, b}^{\dagger}\right\rangle \\
& \left.-\frac{1}{2} \varepsilon_{a c} \varepsilon_{b e} \sigma_{c d}^{\alpha} \sigma_{e f}^{\beta}\left\langle t_{i n \alpha}^{\dagger} t_{i, n+1, \beta}^{\dagger} h_{1 i n d} h_{2 i, n+1, f}\right\rangle\right\} \\
& =\left(\bar{d}^{2}-\frac{1}{2} \bar{s}^{2}-Q_{\perp}\right) \Gamma_{\perp}-\sqrt{2} \bar{d} \bar{s} \tilde{\Pi}_{\perp} \quad(\mathrm{C} 44)
\end{aligned}
$$

where $Q_{\perp}$ and $\Gamma_{\perp}$ are defined previously, and $\tilde{\Pi}_{\perp}$ is given by:

$$
\tilde{\Pi}_{\perp}=-\frac{1}{2} \iint \frac{d k d q}{\pi^{2}} \cos q\left\{\frac{\tilde{\epsilon}_{+}(k, q)}{\tilde{\Omega}_{+}(k, q)}+\frac{\tilde{\epsilon}_{-}(k, q)}{\tilde{\Omega}_{-}(k, q)}\right\} .
$$

\section{APPENDIX D: EFFECT OF COULOMB INTERACTION}

This section is devoted to the effect of Coulomb repulsion. Hamiltonian due to the Coulomb interaction between the nearest neighbors is written in terms of the bond operators as follows.

$$
\begin{aligned}
H_{\text {Coulomb }} & =V \sum_{i} \sum_{n} n_{1 i n} n_{2 i n} \\
& +V \sum_{\langle i, j\rangle} \sum_{n}\left\{n_{1 i n} n_{1 j n}+n_{2 i n} n_{2 j n}\right\} \\
& +V \sum_{i} \sum_{\langle n, m\rangle} n_{2 i n} n_{1 i m}
\end{aligned}
$$

where

$$
\begin{aligned}
n_{1 i n} & \equiv c_{1 \text { ina }}^{\dagger} c_{1 i n a} \\
& =h_{1 i n a}^{\dagger} h_{1 i n a}+s_{i n}^{\dagger} s_{i n}+t_{i n \alpha}^{\dagger} t_{i n \alpha} \\
& =1-h_{2 i n a}^{\dagger} h_{2 i n a}-d_{i n}^{\dagger} d_{i n}
\end{aligned}
$$

and 


$$
\begin{aligned}
n_{2 i n} & \equiv c_{2 i n a}^{\dagger} c_{2 i n a} \\
& =h_{2 i n a}^{\dagger} h_{2 i n a}+s_{i n}^{\dagger} s_{i n}+t_{i n \alpha}^{\dagger} t_{i n \alpha} \\
& =1-h_{1 i n a}^{\dagger} h_{1 i n a}-d_{i n}^{\dagger} d_{i n} .
\end{aligned}
$$

The first term is evaluated as follows.

$$
\begin{aligned}
& \sum_{i} \sum_{n} n_{1 i n} n_{2 i n} \\
= & \sum_{i} \sum_{n}\left(1-h_{2 i n a}^{\dagger} h_{2 i n a}-d_{i n}^{\dagger} d_{i n}\right) \\
& \times\left(1-h_{1 i n b}^{\dagger} h_{1 i n b}-d_{i n}^{\dagger} d_{i n}\right) \\
= & \sum_{i} \sum_{n}\left\{1-d_{i n}^{\dagger} d_{i n}-\left(h_{1 i n a}^{\dagger} h_{1 i n a}+h_{2 i n a}^{\dagger} h_{2 i n a}\right)\right\} \\
= & \sum_{i} \sum_{n}\left\{1-2 x+d_{i n}^{\dagger} d_{i n}\right\} \\
\Rightarrow & \frac{N^{2}}{2}\left(1-2 x+\bar{d}^{2}\right)
\end{aligned}
$$

where $N^{2} / 2$ is the total number of dimers. Note that the constraint conditions are used.

The second term is due to the Coulomb interaction between the dimers along the ladder direction, which is computed by:

$$
\begin{aligned}
& \sum_{\langle i, j\rangle} \sum_{n}\left\{n_{1 i n} n_{1 j n}+n_{2 i n} n_{2 j n}\right\} \\
\Rightarrow & \sum_{\langle i, j\rangle} \sum_{n}\left\{\left(1-\bar{d}^{2}-h_{2 i n a}^{\dagger} h_{2 i n a}\right)\left(1-\bar{d}^{2}-h_{2 j n b}^{\dagger} h_{2 j n b}\right)\right. \\
+ & \left.\left(1-\bar{d}^{2}-h_{1 i n a}^{\dagger} h_{1 i n a}\right)\left(1-\bar{d}^{2}-h_{1 j n b}^{\dagger} h_{1 j n b}\right)\right\} \\
= & N^{2}\left(1-\bar{d}^{2}\right)\left(1-2 x+\bar{d}^{2}\right) \\
+ & \sum_{\langle i, j\rangle} \sum_{n}\left\{h_{1 i n a}^{\dagger} h_{1 i n a} h_{1 j n b}^{\dagger} h_{1 j n b}+h_{2 i n a}^{\dagger} h_{2 i n a} h_{2 j n b}^{\dagger} h_{2 j n b}\right\} .
\end{aligned}
$$

Similarly, the third term is evaluated as follows.

$$
\begin{aligned}
& \sum_{i} \sum_{\langle n, m\rangle} n_{2 i n} n_{1 i m} \\
= & \frac{N^{2}}{2}\left(1-\bar{d}^{2}\right)\left(1-2 x+\bar{d}^{2}\right) \\
+ & \sum_{i} \sum_{\langle n, m\rangle} h_{1 i n a}^{\dagger} h_{1 i n a} h_{2 i m b}^{\dagger} h_{2 i m b}
\end{aligned}
$$

Therefore,

$$
\begin{aligned}
& H_{\text {Coulomb }}=\frac{N^{2}}{2} V\left(4-3 \bar{d}^{2}\right)\left(1-2 x+\bar{d}^{2}\right) \\
& +V \sum_{\langle i, j\rangle} \sum_{n}\left\{h_{1 \text { ina }}^{\dagger} h_{1 i n a} h_{1 j n b}^{\dagger} h_{1 j n b}+h_{2 i n a}^{\dagger} h_{2 i n a} h_{2 j n b}^{\dagger} h_{2 j n b}\right\} \\
& +V \sum_{i} \sum_{\langle n, m\rangle} h_{1 i n a}^{\dagger} h_{1 i n a} h_{2 i m b}^{\dagger} h_{2 i m b} \\
& =\frac{N^{2}}{2} V\left(4-3 \bar{d}^{2}\right)\left(1-2 x+\bar{d}^{2}\right)+H_{C o u l, h^{4}}
\end{aligned}
$$

The full treatment of $H_{C o u l, h^{4}}$ is beyond the scope of our present paper. However, we will follow the philosophy of BCS theory in that electrons interacting with the Coulomb repulsion under the neutralizing background are described in terms of the Landau-Fermi liquid. Assuming that the hopping parameter, $t$, and the spincoupling parameter, $J$, are already renoramilzed quatities due to the Coulomb repulsion, one has only to consider the Hartree contribution of $H_{C o u l}, h^{4}$, which is given by:

$$
H_{\text {Coul }, h^{4}}^{\text {Hartree }}=\frac{N^{2}}{2} 3 V\left(x-\bar{d}^{2}\right)^{2}
$$

which is obtained by using the constraint conditions. Also, note that the mixing between the bonding and antibonding fermions is ignored.

Therefore, in our approximation, the Hamiltonian (per site) due to the Coulomb repulsion is given as follows:

$$
\begin{aligned}
\frac{H_{\text {Coulomb }}}{N^{2} / 2} & \simeq V\left(4-3 \bar{d}^{2}\right)\left(1-2 x+\bar{d}^{2}\right)+\text { HCoul }_{\text {Ch }}^{\text {Hartree }} \\
& =V\left(4-8 x+3 x^{2}\right)+V \bar{d}^{2}
\end{aligned}
$$

Since Eq.(D9) depends only on $\bar{d}^{2}$, the modification of saddle-point equations is straightforward. That is,

$$
\frac{\partial\left(\epsilon_{g r} / J\right)}{\partial \bar{d}^{2}}=0 \rightarrow \frac{\partial\left(\epsilon_{g r} / J\right)}{\partial \bar{d}^{2}}+V=0
$$

All the other saddle-point equations are the same as before.

Now it is reasonable to assume the limit of large Coulomb repulsion which not only captures the essential physics that we are interested in, but also will simplify explicit computations. So we will take the limit $V \rightarrow \infty$; consequently $\bar{d}=0$. While this limit is not physical for large doping, our primary interest lies in the case of small doping.

\footnotetext{
${ }^{1}$ See e.g. S. Sachdev, Science, 288, 475 (2000) and references therein.

${ }^{2}$ J. Bobroff, H. Alloul, W. A. MacFarlane, P. Mendels, N. Blanchard, G. Collin, and J.-F. Marucco, Phys. Rev. Lett. 86, 4116 (2001).

${ }^{3}$ H. Alloul et al., Phys. Rev. Lett. 67, 3140 (1991); A. V. Mahajan et al., Phys. Rev. Lett. 72, 3100 (1994); J. Bobroff et al., Phys. Rev. Lett. 83, 4381 (1999); P. Mendels et al., Europhys. Lett. 46, 678 (1999); A. V. Mahajan et al. Eur. Phys. J. B 13, 457 (2000); M.-H. Julien et al., Phys. Rev. Lett. 84, 3422 (2000).

${ }^{4}$ N. Read and S. Sachdev, Phys. Rev. Lett. 62, 1694 (1989); Phys. Rev. B 42, 4568 (1990).
}

${ }^{5}$ S. Sachdev and N. Read, Int. J. Mod. Phys. B 5, 219 (1991). 
${ }^{6}$ S. Sachdev and R. Jalabert, Mod. Phys. Lett. B 4, 1043 (1990).

${ }^{7}$ M. Vojta and S. Sachdev, Phys. Rev. Lett. 83, 3916 (1999); M. Vojta, Y. Zhang, and S. Sachdev, Phys. Rev. B 62, 6721 (2000).

${ }^{8}$ S. Sachdev, C. Buragohain, and M. Vojta, Science 286, 2479 (1999); M. Vojta, C. Buragohain, and S. Sachdev, Phys. Rev. B 61, 15152 (2000).

${ }^{9}$ S. Sachdev and M. Vojta, Physica B 280, 333 (2000).

$10 \mathrm{~S}$. Sachdev and M. Vojta, Proceedings of the XIII International Congress on Mathematical Physics, July 2000, London, cond-mat/0009202.

${ }^{11}$ E. Fradkin and S. Kivelson, Mod. Phys. Lett. B 4, 225 (1990).

${ }^{12}$ H. Tsunetsugu, M. Troyer, and T. M. Rice, Phys. Rev. B 49, 16078 (1994); M. Troyer, H. Tsunetsugu, and T. M. Rice, Phys. Rev. B 53, 251 (1996).

${ }^{13}$ D. Poilblanc, O. Chiappa, J. Riera, S. R. White, and D. J. Scalapino, cond-mat/0005403

14 J. Rossat-Mignod, L. P. Regnault, C. Vettier, P. Bourges, P. Burlet, J. Bossy, J. Y. Henry, and G. Lapertot, Physica C 185-189, 86 (1991).

${ }^{15}$ H. A. Mook, M. Yethiraj, G. Aeppli, T. E. Mason, and T. Armstrong, Phys. Rev. Lett. 70, 3490 (1993).

${ }^{16}$ H. F. Fong, B. Keimer, D. Reznik, D. L. Milius, and I. A. Aksay, Phys. Rev. B 54, 6708 (1996); H. F. Fong, B. Keimer, D. L. Milius, and I. A. Aksay, Phys. Rev. Lett. 78, 713 (1997).

${ }^{17} \mathrm{P}$. Bourges in The Gap Symmetry and Fluctuations in High Temperature Superconductors ed. J. Bok, G. Deutscher, D. Pavuna, and S. A. Wolf (Plenum, New York, 1998); cond-mat/9901333.

18 H. He, Y. Sidis, P. Bourges, G. D. Gu, A. Ivanov, N. Koshizuka, B. Liang, C. T. Lin, L. P. Regnault, E. Schoenherr, and B. Keimer, Phys. Rev. Lett. 86, 1610 (2001).

19 J. M. Tranquada, J. D. Axe, N. Ichikawa, A. R. Moodenbaugh, Y. Nakamura, and S. Uchida, Phys. Rev. Lett. 78, 338 (1997)

${ }^{20}$ V. J. Emery, S. A. Kivelson, J. M. Tranquada, Proc. Natl. Acad. Sci 96, 8814 (1999) and references therein.

${ }^{21}$ R. J. McQueeney, Y. Petrov, T. Egami, M. Yethiraj, G. Shirane, and Y. Endoh, Phys. Rev. Lett. 82, 628 (1999); T. Egami, cond-mat/0102449; R. J. McQueeney, J. L. Sarrao, P. G. Pagliuso, P. W. Stephens, and R. Osborn, condmat/0104118; R. J. McQueeney, T. Egami, J.-H. Chung, Y. Petrov, M. Yethiraj, M. Arai, Y. Inamura, Y. Endoh, C. Frost, and F. Dogan, cond-mat/0105593.

${ }^{22}$ D. Withoff and E. Fradkin, Phys. Rev. Lett. 64, 1835 (1990); L. S. Borkowski and P. J. Hirschfeld, Phys. Rev. B 46, 9274 (1992); K. Chen and C. Jayaprakash, J. Phys.: Condens. Matter 7, L491 (1995); K. Ingersent, Phys. Rev. B 54, 11936 (1996); C. R. Cassanello and E. Fradkin, Phys. Rev. B 53, 15079 (1996) and 56, 11246 (1997); R. Bulla, Th. Pruschke, and A. C. Hewson, J. Phys.: Condens. Matter 9, 10463 (1997); K. Ingersent and Q. Si, condmat/9810226; C. Gonzalez-Buxton and K. Ingersent, Phys. Rev. B 57, 14254 (1998).

${ }^{23}$ A. Polkovnikov, S. Sachdev, and M. Vojta, Phys. Rev. Lett. 86, 296 (2001).
24 J-X. Zhu and C. S. Ting, cond-mat/0008156.

${ }^{25}$ A. V. Chubukov, S. Sachdev, and J. Ye, Phys. Rev. B 49 11919 (1994).

${ }^{26}$ N. Nagaosa and P. A. Lee, Phys. Rev. B 61, 9166 (2000).

${ }^{27}$ S. Sachdev and R. N. Bhatt, Phys. Rev. B 41, 9323 (1990).

${ }^{28}$ A. V. Chubukov and Th. Jolicoeur, Phys. Rev. B 44, 12050 (1991)

29 T. M. Rice, S. Gopalan, M. Sigrist, Europhys. Lett. 23, 445 (1993).

${ }^{30}$ S. Gopalan, T. M. Rice, and M. Sigrist, Phys. Rev. B 49, 8901 (1994).

31 J Piekarewicz and J. R. Shepard, Phys. Rev. B 60, 9456 (1999).

${ }^{32}$ V. N. Kotov, O. Sushkov, Zheng Weihong, and J. Oitmaa, Phys. Rev. Lett. 80, 5790 (1998); V. N. Kotov, J. Oitmaa, O. Sushkov, and Zheng Weihong, Phil. Mag. B 80, 1483 (2000).

${ }^{33}$ Y. Matsushita, M. P. Gelfand, and C. Ishii, J. Phys. Soc. Jpn., 68247 (1999).

${ }^{34}$ K. Totsuka, S. Miyahara, K. Ueda, Phys. Rev. Lett. 86, 520 (2001).

${ }^{35}$ D. Carpentier and L. Balents, cond-mat/0102218.

${ }^{36}$ R. Eder, A. Dorneich, M. G. Zacher, W. Hanke, and S.C. Zhang, Phys. Rev. B 59, 561 (1999); A. Furusaki, S.C. Zhang, Phys. Rev. B 60, 1175 (1999).

${ }^{37}$ E. Demler and S. Das Sarma, Phys. Rev. Lett. 82, 3895 (1999).

38 T. Sommer, M. Vojta, and K. W. Becker, condmat/0104356

${ }^{39}$ Y. L. Lee, Y. W. Lee, C.-Y. Mou, and Z. Y. Weng, Phys. Rev. B 60, 13418 (1999).

${ }^{40}$ R. Eder, Phys. Rev. B 57, 12832 (1998).

${ }^{41}$ O. P. Sushkov, cond-mat/0002421.

${ }^{42}$ M. Vojta and K. W. Becker, Phys. Rev. B 60, 15201 (1999).

${ }^{43}$ C. Jurecka and W. Brenig, cond-mat/0103511.

${ }^{44}$ S. Sachdev, Quantum Phase Transitions, Cambridge University Press, Cambridge (1999).

${ }^{45}$ M. G. Zacher, R. Eder, E. Arrigoni, and W. Hanke, condmat/0103030

${ }^{46}$ M. Granath, V. Oganesyan, S. A. Kivelson, E. Fradkin, and V. J. Emery, cond-mat/0010350

${ }^{47}$ R. D. Duncan and C. A. R. Sá de Melo, Phys. Rev. B 62, $9675(2000)$

${ }^{48}$ C. Nayak, Phys. Rev. B 62, 4880 (2000).

${ }^{49}$ L. Pintschovius and M. Braden, Phys. Rev. B 60, R15039 (1999).

${ }^{50}$ H. A. Mook and F. Dogan, Nature 401, 145 (1999); H. A. Mook, P. Dai, F. Dogan, and R. D. Hunt, Nature 404, 729 (2000).

${ }^{51}$ Y. Petrov, T. Egami, R. J. McQueeney, M. Yethiraj, H. A. Mook, and F. Dogan, cond-mat/0003414.

${ }^{52}$ G. Khaliullin and P. Horsch, Physica C 282-287, 1751 (1997); P. Horsch, G. Khaliullin, and V. Oudovenko, Physica C 341-348, 117 (2000).

${ }^{53}$ C. Gros and R. Werner, Phys. Rev. B 58, R14677 (1998).

${ }^{54}$ R. Werner, C. Gros, and M. Braden, Phys. Rev. B 59, 14356 (1999).

${ }^{55}$ M. J. Massey, R. Merlin, and S. M. Girvin, Phys. Rev. Lett. 69, 2299 (1992).

${ }^{56}$ Z.-X. Shen, A. Lanzara, and N. Nagaosa, cond- 
mat/0102224.

${ }^{57}$ G. L. Squires, Introduction to the Theory of Thermal Neutron Scattering, Dover Publications, New York (1978); see also http://rrdjazz.nist.gov/resources/n-lengths/.

${ }^{58}$ S. Ono, Yoichi Ando, T. Murayama, F. F. Balakirev, J. B. Betts, and G. S. Boebinger, Phys. Rev. Lett. 85, 638 (2000).

${ }^{59}$ E. Demler, S. Sachdev, and Y. Zhang, Phys. Rev. Lett. 87, 067202 (2001).

${ }^{60}$ J. C. Wynn, D. A. Bonn, B. W. Gardner, Yu-Ju Lin, Ruixing Liang, W. N. Hardy, J. R. Kirtley, and K. A. Moler, preprint.

${ }^{61}$ S. Sachdev, Phys. Rev. B 45, 389 (1992); N. Nagaosa and P. A. Lee, Phys. Rev. B 45, 966 (1992); Jung Hoon Han and Dung-Hai Lee, Phys. Rev. Lett. 85, 1100 (2000); M. Franz and Z. Tesanovic, Phys. Rev. B 63, 064516 (2001).

${ }^{62}$ Ch. Renner, B. Revaz, K. Kadowaki, I. Maggio-Aprile, and O. Fischer, Phys. Rev. Lett. 80, 3606 (1998); S. H. Pan, E. W. Hudson, A. K. Gupta, K.-W. Ng, H. Eisaki, S. Uchida, and J. C. Davis, Phys. Rev. Lett. 85, 1536 (2000).

${ }^{63}$ S. H. Pan, J. P. O'Neal, R .L. Badzey, C. Chamon, H. Ding, J. R. Engelbrecht, Z. Wang, H. Eisaki, S. Uchida, A. K. Gupta, K.-W. Ng, E. W. Hudson, K. M. Lang, and J. C. Davis, preprint.

${ }^{64}$ O. P. Sushkov, J. Oitmaa, and Zheng Weihong, Phys. Rev. B 63, 104420 (2001).

${ }^{65}$ M. S. L. du Croo de Jongh, J. M. J. van Leeuwen, W. van Saarloos, Phys. Rev. B 62, 14844 (2000).

${ }^{66}$ S. Mazumdar, S. Ramasesha, R. T. Clay, and D. K. Campbell, Phys. Rev. Lett. 82, 1522 (1999).

67 S. Mazumdar, R. T. Clay, and D. K. Campbell, condmat/9910164.

${ }^{68}$ S. Mazumdar, R. T. Clay, and D. K. Campbell, Phys. Rev. B 62, 13400 (2000).

${ }^{69}$ S. A. Kivelson, E. Fradkin, and V. J. Emery, Nature 393, 550 (1998).

${ }^{70}$ V. J. Emery, S. A. Kivelson, and O. Zachar, Phys. Rev. B 56, 6120 (1997).

${ }^{71}$ S. Sachdev, Phys. Rev. B 40, 5204 (1989).

${ }^{72}$ E. W. Carlson, D. Orgad, S. A. Kivelson, and V. J. Emery, Phys. Rev. B 62, 3422 (2000).

${ }^{73}$ J. Zaanen, O. Y. Osman, H. V. Kruis, Z. Nussinov, and J. Tworzydlo, cond-mat/0102103.

${ }^{74}$ M. Vojta, Y. Zhang, and S. Sachdev, Phys. Rev. Lett. 85, 4940 (2000); Int. J. Mod. Phys. B 14, 3719 (2000).

${ }^{75}$ S. R. White and D. J Scalapino, Phys. Rev. Lett. 80, 1272 (1998); 81, 3227 (1998); Phys. Rev. B 60, R753 (1999).

76 A. H. Castro Neto, cond-mat/0102281

77 C. Lannert, M. P. A. Fisher, and T. Senthil, Phys. Rev. B 63, 134510 (2001).

${ }^{78}$ J. V. Jose, L. P. Kadanoff, S. Kirkpatrick, and D. R. Nelson, Phys. Rev. B 16, 1217 (1977). 